Approved for public release; distribution is untimited.

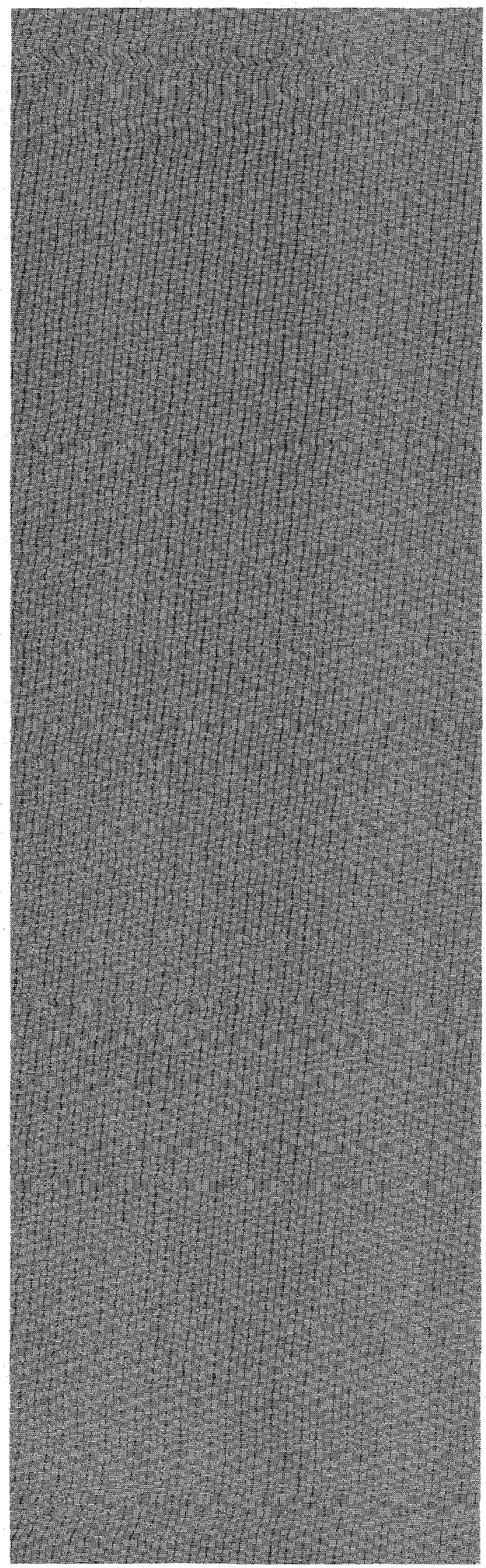

\section{Open Cycle Thermoacoustics}

\section{Los Alamos \\ NATIONAL LABORATORY}

Los Alamos National Laboratory is operated by the University of California for the United States Department of Energy under contract W-7405-ENG-36. 
This thesis was accepted by the School of Mechanical Engineering, Georgia Institute of Technology, Atlanta, Georgia, in partial fulfillment of the requirements for the degree of Doctor of Philosophy. The text and illustrations are the independent work of the author and only the front matter has been edited by the CIC-1 Writing and Editing Staff to conform with Department of Energy and Los Alamos National Laboratory publication policies.

\section{An Affirmative Action/Equal Opportunity Employer}

This report was prepared as an account of work sponsored by an agency of the United States Government. Neither The Regents of the University of California, the United States Government nor any agency thereof, nor any of their employees, makes any warranty, express or implied, or assumes any legal liability or responsibility for the accuracy, completeness, or usefulness of any information, apparatus, product, or process disclosed, or represents that its use would not infringe privately owned rights. Reference herein to any specific commercial product, process, or service by trade name, trademark, manufacturer, or otherwise, does not necessarily constitute or imply its endorsement, recommendation, or favoring by The Regents of the University of California, the United States Government, or any agency thereof. The views and opinions of authors expressed herein do not necessarily state or reflect those of The Regents of the University of California, the United States Government, or any agency thereof. Los Alamos National Laboratory strongly supports academic freedom and a researcher's right to publish; as an institution, howeoer, the Laboratory does not endorse the vieropoint of a publication or guarantee its technical correctness. 


\section{DISCLAIMER}

Portions of this document may be illegible in electronic image products. Images are produced from the best available original document. 


\section{Open Cycle Thermoacoustics}

Robert Stowers Reid 



\section{ACKNOWLEDGMENTS}

I would like to thank my thesis advisor Professor P. V. Desai for his ready help, wealth of sound advice, and guidance through the completion of this thesis.

Open cycle thermoacoustics is the brainchild of Greg Swift. Thanks, Greg, for letting me run with your idea as far as I could take it. You more than held your end of the bargain. I can only hope that I held mine.

I would like to acknowledge the indispensable support provided by Chris Espinoza in the fabrication and assembly of this experiment. I would also like to acknowledge Bill Ward who greatly simplified my life with a rational design for the loudspeaker system used in this experiment as well as timely updates to the linear acoustic model.

Professor Gene Colwell gave much wise counsel during my year at Georgia Tech.

Paul Wantuck and Gary Read provided discretionary financial support during the latter stages of my research.

Finally, I'd like to thank Mike Merrigan for continued professional support and advice during my entire career at Los Alamos.

This work was funded by the US Department of Energy's Office of Basic Energy Sciences.

Since the most common problem the builder of machines meets is to extract from given resources the maximum power, with the machinery to be used as the variable under his control, this maximum utilization of power is set up as an absolute ideal, a value itself. But there is, of course, no special merit economizing one of the many factors which limit the possible achievement, at the expense of others. The engineer's "technical optimum" proves frequently to be simply that method which it would be desirable to adopt if the supply of capital were unlimited, or the rate of interest were zero.

F.A. Hayek, The Counter-Revolution of Science, 1952. 


\section{CONTENTS}

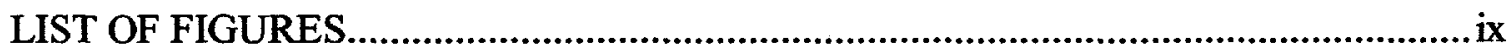

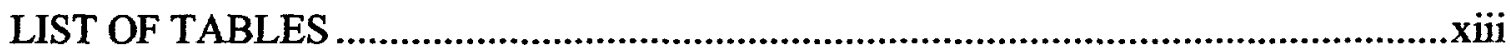

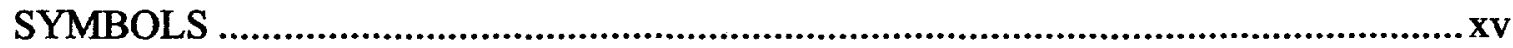

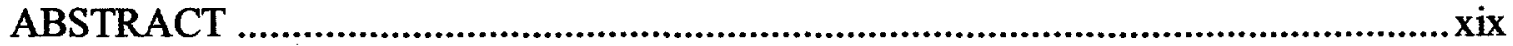

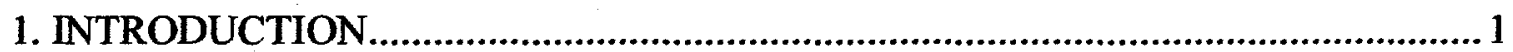

Description of Conventional Thermoacoustic Devices............................................4

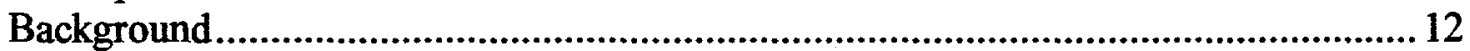

2. SOME FEATURES OF OPEN CYCLE THERMOACOUSTICS......................... 16

Nondimensional Forms of the Governing Equations ..............................................2.22

Inviscid Short Stack Approximation .............................................................. 33

Dimensionless Enthalpy-Flux Ratios ............................................................... 42

Cycle Representation in Phase Space............................................................ 45

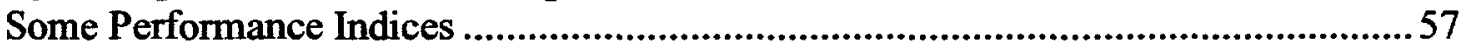

3. APPLIED FLOW REFRIGERATOR AND EXPERIMENT ...............................62

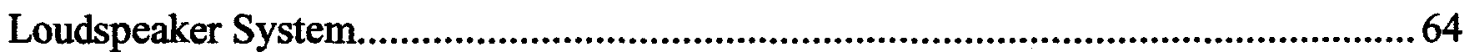

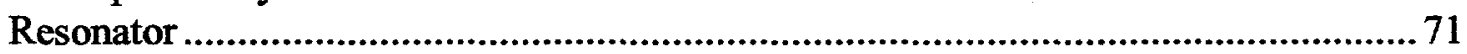

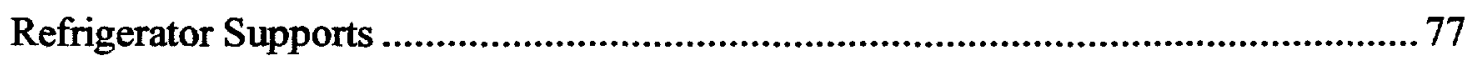

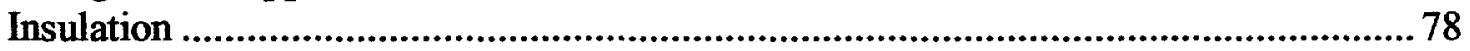

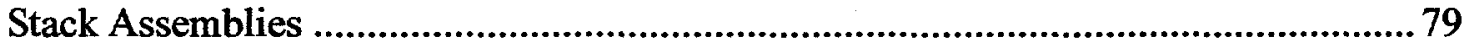

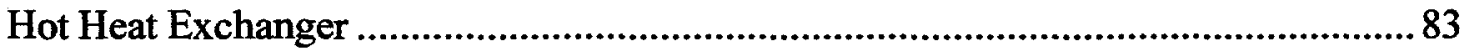

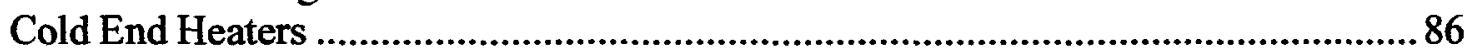

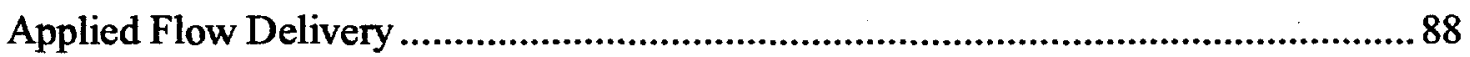

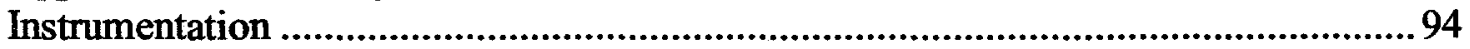

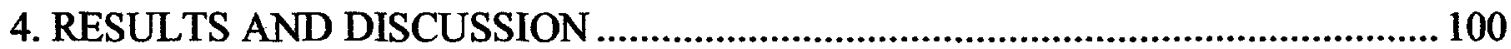

Stack Performance with Position ...................................................................... 105

Stack Performance at Various Applied Flow Rates ............................................. 111

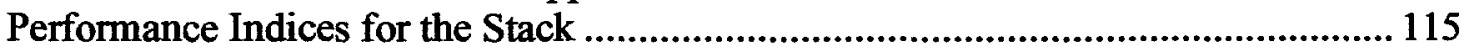

5. CONCLUSIONS AND SUGGESTIONS FOR FUTURE WORK ........................ 129

A. PROPOSED EQUATIONS FOR APPLIED FLOW THROUGH STACK.............. 133

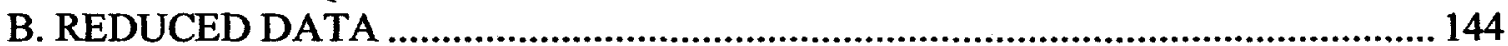

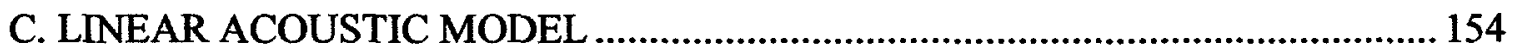

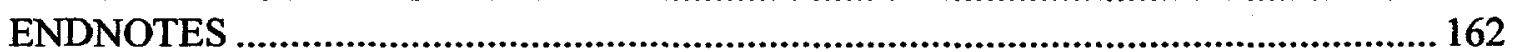

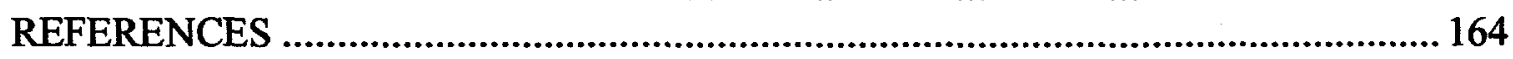

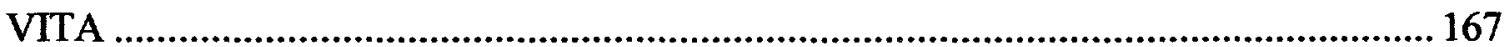




\section{LIST OF FIGURES}

Figure 1. Examples of thermodynamic cycles using a gaseous working fluid.

(a) An open thermodynamic process: the Lagrangian path in temperature-entropy space followed by an air-cycle refrigeration system found in jet aircraft. (b) A closed thermodynamic cycle: the Lagrangian $T-s$ path followed by a gas turbine power plant. (c) The superposition of an open process on a closed cycle: the Lagrangian $T-s$ path followed by an open thermoacoustic refrigerator.

Figure 2. Closed-cycle thermoacoustic devices............................................................6

Figure 3. Lagrangian view of the work-heat interaction for a gas parcel near a thermoacoustic stack plate in a simplified thermoacoustic engine cycle.

Figure 4. Lagrangian view of the work-heat interaction for a gas parcel near a thermoacoustic stack plate in a simplified thermoacoustic refrigeration cycle.

Figure 5. Comparison of four different half-wavelength open-cycle thermoacoustic devices.

Figure 6. $T-x$ coordinate system for an open-cycle thermoacoustic refrigerator

Figure 7. $T-x$ coordinate system for an open-cycle thermoacoustic heater

Figure 8. $T-s$ representation of an inviscid open thermoacoustic refrigeration cycle. 
Figure 9. $T-x$ representation of an inviscid open thermoacoustic refrigeration cycle.

Figure 10. $s-x$ representation of an inviscid open thermoacoustic refrigeration cycle.

Figure 11. Schematic of a full wavelength thermoacoustic refrigerator with an externally applied flow. Labeled " $N$ " are the locations of the pressure nodes of the standing wave. " $H$ " indicates a room temperature heat exchanger and " $C$ " indicates a cold heat exchanger. The entrance and exit of the applied flow for the refrigerator is indicated by heavy arrows near the nodes. The cold heat exchangers are unnecessary for cooling the gas.

Figure 12. Domain and key variables for (a) resonator with a diffuser and (b) its mathematical representation. .74

Figure 13. Scale drawing of stack showing end and lateral views through the central cross section. 82

Figure 14. End and lateral views of hot heat exchanger. All dimensions are to scale, except that the fin spacing has been exaggerated by a factor of two for clarity. .84

Figure 15. Scale drawing of cold heat exchanger showing an end view and two lateral cross sections. For clarity, the end view has the top section removed.

Figure 16. Schematic showing applied flow control, delivery, and measurement systems.

Figure 17. Temperature sensor placement...............................................................95

Figure 18. Pressure sensor placement. ........................................................................97

Figure 19. Acoustic power delivered to the refrigerator as a function of applied volumetric flow rate. 
Figure 20. Right stack temperature versus distance from the hot end at various applied volumetric flow rates. The symbols are measurements. The line traces show the corresponding numerical results based on the acoustic model. 106

Figure 21. Left stack temperature versus distance from the hot end at various applied volumetric flow rates. The symbols are measurements. The line traces show the corresponding numerical results based on the acoustic model.

Figure 22. Right stack total refrigeration power versus applied volumetric flow rate. All data were taken with $\left|p_{1}\right| p_{0}=0.0200, T_{H}=308 \mathrm{~K}$, and $T_{C}=300.5 \mathrm{~K}$. Symbols are measurements: $\mathrm{O}-\Delta \dot{H}_{0}$, $-\dot{Q}_{c}, \square-\dot{Q}_{c}+\Delta \dot{H}_{0}$ (refrigerator), $\nabla-\dot{Q}_{H}, \square-$ $\dot{Q}_{H}+\Delta \dot{H}_{0}$ (heater), lines are the corresponding acoustic model calculations.

Figure 23. Left stack total refrigeration power versus applied volumetric flow rate. All data were taken with $\left|p_{1}\right| p_{0}=0.0200, T_{H}=308 \mathrm{~K}$, and $T_{C}=300.5 \mathrm{~K}$. Symbols are measurements: $\mathrm{O}-\Delta \dot{H}_{0}$, $-\dot{Q}_{c}, \square-\dot{Q}_{c}+\Delta \dot{H}_{0}$ (refrigerator), $\nabla-\dot{Q}_{H}, \square-$ $\dot{Q}_{H}+\Delta \dot{H}_{0}$ (heater), lines are the corresponding acoustic model calculations.

Figure 24. Left stack performance indices versus dimensionless applied volumetric flow rate. The symbols represented by $O$ were calculated with Equation (79). The symbols represented by were calculated with Equation (80).

Figure 25. Right stack performance indices versus dimensionless applied volumetric flow rate. The symbols represented by $O$ were 
calculated with Equation (79). The symbols represented by were calculated with Equation (80).

Figure 26. Comparison of single stage to multi-stage modes of operation for the devices previously defined in Figure 5: "Device B" open cycle heater and the "Device A" open cycle refrigerator.

Figure 27. Ratio of the open cycle performance index to the closed cycle performance index versus dimensionless applied volumetric flow rate for the left stack. The symbols - and $\boldsymbol{a}$, represent the heater and refrigerator data defined by Equations (84) and (81), respectively. The symbols $O$ and $\square$, represent the ideal results given by Equations (85) and (83), respectively. 123

Figure 28. Same results as shown in Figure 27, but for the right stack. 124

Figure 29. Fractional Carnot efficiency (Second-Law effectiveness) for the left stack versus dimensionless applied volumetric flow rate. For velocities $\geq 0$ a refrigerator performance index, defined by Equation (86) and represented by the symbol $\bullet$ is used, while for velocities $\leq 0$ a heater performance index, defined by Equation (87) and represented by $\mathbf{m}$ is used. 126

Figure 30. Fractional Carnot efficiency (Second-Law effectiveness) for the right stack versus dimensionless applied volumetric flow rate. For velocities $\geq 0$ a refrigerator performance index, defined by Equation (86) and represented by the symbol $\bullet$ is used, while for velocities $\leq 0$ a heater performance index, defined by Equation (87) and represented by $\mathbf{\square}$ is used.

Figure 31. A symmetry relation found in the exponential temperature profiles among the four types of open-cycle thermoacoustic device defined in Chapter 2.

Figure 32. Configuration used in linear acoustic model, DeltaE 155 


\section{LIST OF TABLES}

Table 1. List of Some Dimensionless Variables........................................................28

Table 2. Inviscid Short Stack Assumptions.............................................................41

Table 3. Representative Refrigerator Stack Conditions ...................................................51

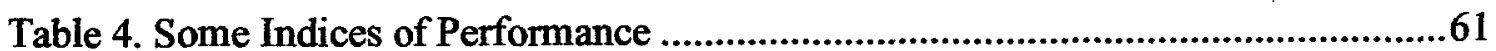

Table B.1. Variables Listed in Reduced Data..............................................................145

Table B.2. Variables Listed in System Performance Measures..................................... 146

Table B.3. Enthalpy Flux Ratio Definitions .................................................................147

Table B.4. Reduced Data on Left Side of Resonator ................................................... 148

Table B.5. Reduced Data on Right Side of Resonator ....................................................149

Table B.6. Estimated Uncertainty for Left Side Reduced Data....................................150

Table B.7. Estimated Uncertainty for Right Side Reduced Data....................................151

Table B.8. System Performance Measures..................................................................152

Table B.9. Values for Various Enthalpy Flux Ratios....................................................153 


\section{SYMBOLS}

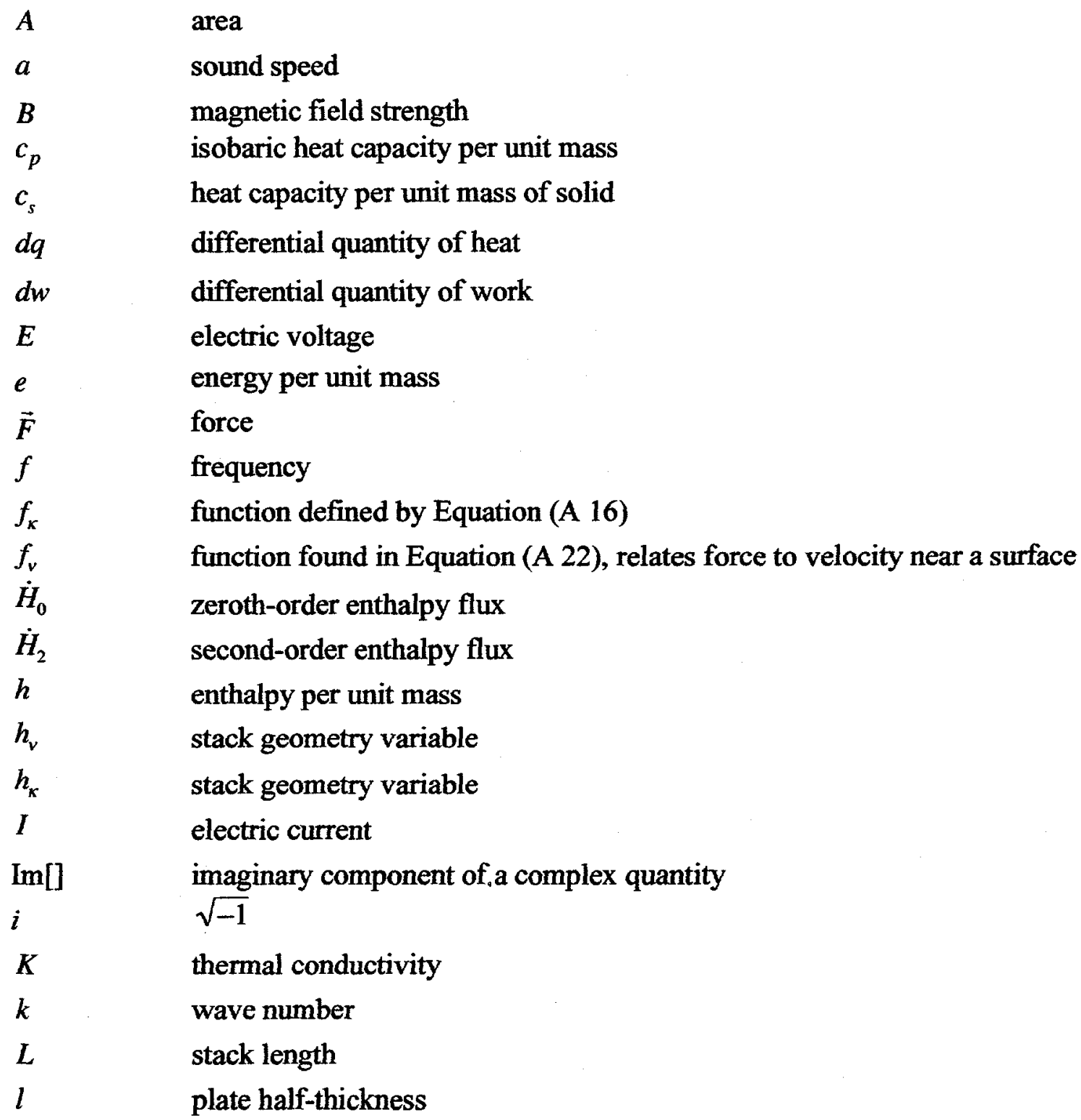




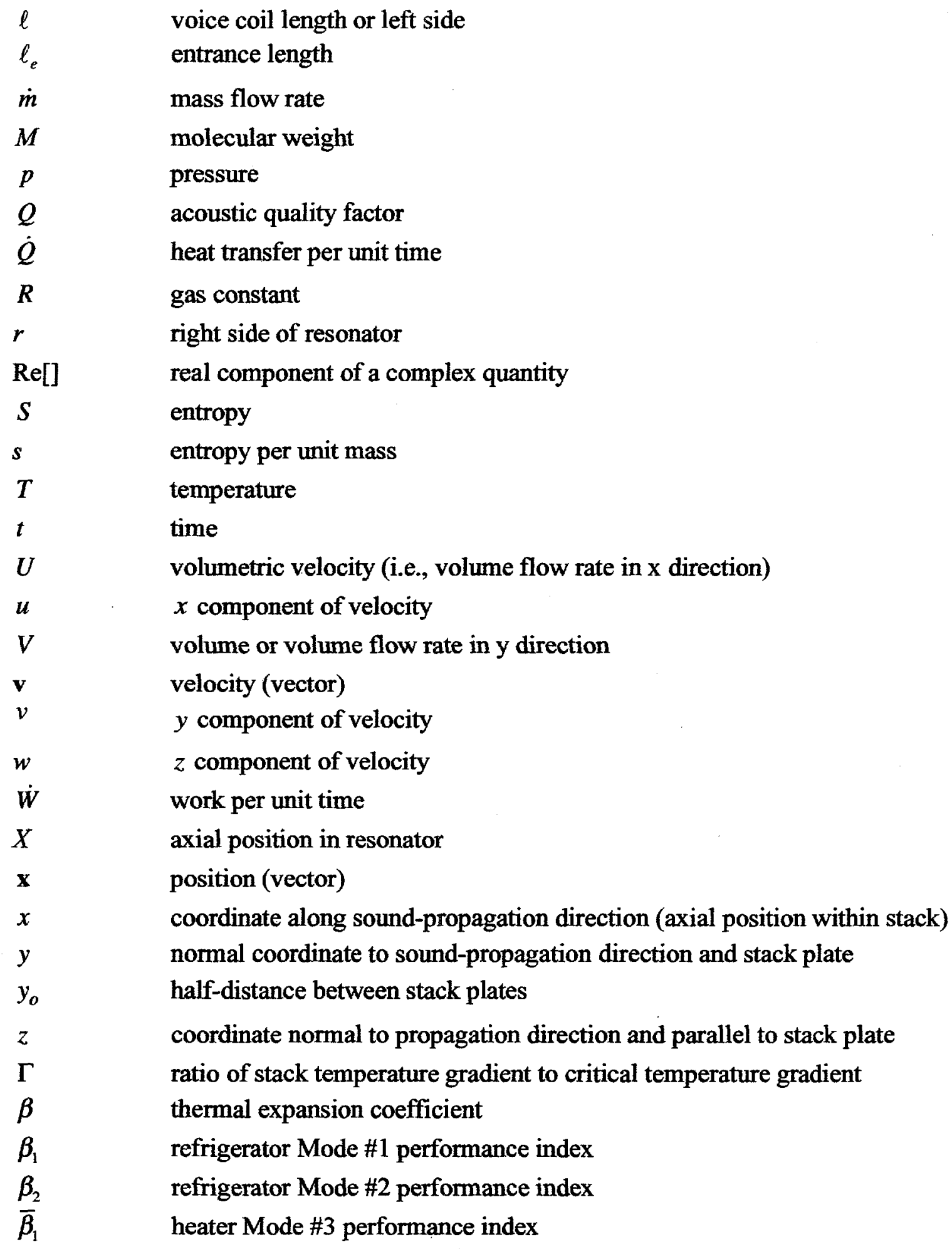




\begin{tabular}{ll}
$\bar{\beta}_{2}$ & heater Mode \#4 performance index \\
$\gamma$ & ratio: isobaric to isochoric specific heats \\
$\delta$ & penetration depth or Kronecker delta \\
$\varepsilon$ & Second Law effectiveness \\
$\zeta$ & Lagrangian displacement of fluid particle or second viscosity \\
$\eta$ & efficiency \\
$\eta_{1}$ & engine Mode \#1 performance index \\
$\eta_{2}$ & engine Mode \#2 performance index \\
$\bar{\eta}_{1}$ & engine-heater \#1 performance index \\
$\overline{\eta_{2}}$ & engine-heater \#2 performance index \\
$\theta$ & dimensionless temperature difference \\
$\kappa$ & thermal diffusivity \\
$\lambda$ & wavelength \\
$\lambda$ & radian wavelength \\
$\mu$ & dynamic viscosity \\
$v$ & kinematic viscosity \\
$\Xi$ & ratio of flow enthalpy flux to thermoacoustic second-order enthalpy flux \\
$\xi$ & dimensionless velocity \\
$\rho$ & density \\
$\Sigma$ & viscous stress (tensor) \\
$\sigma$ & Prandtl number \\
$\tau$ & period \\
$\omega$ & angular frequency \\
\hline
\end{tabular}

Subscripts and superscripts

$\begin{array}{ll}A & \text { amplitude } \\ a & \text { applied } \\ a c & \text { acoustic } \\ \text { C } & \text { Carnot } \\ C & \text { cold } \\ \text { crit } & \text { critical } \\ e & \text { end or exit }\end{array}$




\begin{tabular}{ll} 
elec & electrical \\
G & global \\
$H$ & hot \\
$h$ & hydraulic \\
$h x$ & total power dissipated by hot heat exchanger \\
int & internally reversible \\
$i, k, l$ & indicial indices \\
$k$ & kinetic \\
$L$ & lower portion of resonator \\
$\ell$ & left side \\
min & minimum \\
$n$ & device number indice \\
$p$ & potential \\
$r$ & right side \\
$r e f$ & reference \\
$r e s$ & resonator \\
$r e v$ & reversible \\
S & short stack \\
$s$ & solid (subscript) or standing wave component (superscript) \\
$t$ & transition \\
tot & total \\
$\kappa$ & thermal \\
$v$ & viscous \\
0 & zeroth-order quantity (subscript) no-flow condition (superscript) \\
1 & first-order quantity \\
2 & second-order quantity \\
- & denotes a dimensionless quantity \\
\langle\rangle & denotes a spatially averaged quantity \\
$\sim$ & denotes a quantity taken normal to the sound propagation direction \\
& complex conjugate \\
& \\
\hline &
\end{tabular}




\title{
OPEN CYCLE THERMOACOUSTICS
}

\author{
by
}

Robert Stowers Reid

\begin{abstract}
A new type of thermodynamic device combining a thermodynamic cycle with the externally applied steady flow of an open thermodynamic process is discussed and experimentally demonstrated. The gas flowing through this device can be heated or cooled in a series of semi-open cyclic steps. The combination of open and cyclic flows makes possible the elimination of some or all of the heat exchangers (with their associated irreversibility). Heat is directly exchanged with the process fluid as it flows through the device when operating as a refrigerator, producing a staging effect that tends to increase First Law thermodynamic efficiency. An open-flow thermoacoustic refrigerator was built to demonstrate this concept. Several approaches are presented that describe the physical characteristics of this device. Tests have been conducted on this refrigerator with good agreement with a proposed theory.
\end{abstract}




\section{CHAPTER 1}

\section{INTRODUCTION}

Refrigeration, air conditioning, and prime power systems in widespread use today rely on thermodynamic processes in fluids. Many thermodynamic systems are open, with each mass element of working fluid passing once through its sequence of changes in temperature, pressure, density, and entropy. Both mass and energy (in the form of work and heat) cross the boundary of an open thermodynamic system. Examples of open systems include ramjets, some gas turbines, internal combustion engines, and the Brayton-cycle refrigerator with $T-s$ path depicted in Figure 1(a). Other systems are closed and cyclic, with each mass element of working fluid passing repeatedly through its sequence of thermodynamic steps with only energy crossing its boundary. Such closed systems include Rankine-cycle refrigerators and Stirling machines, and the closed Brayton cycle power plant with $T-s$ path depicted in Figure 1(b).

Closed thermodynamic cycles need at least two heat exchangers for heat transfer between the thermodynamic working fluid (often a chlorofluorocarbon, steam, or helium) 


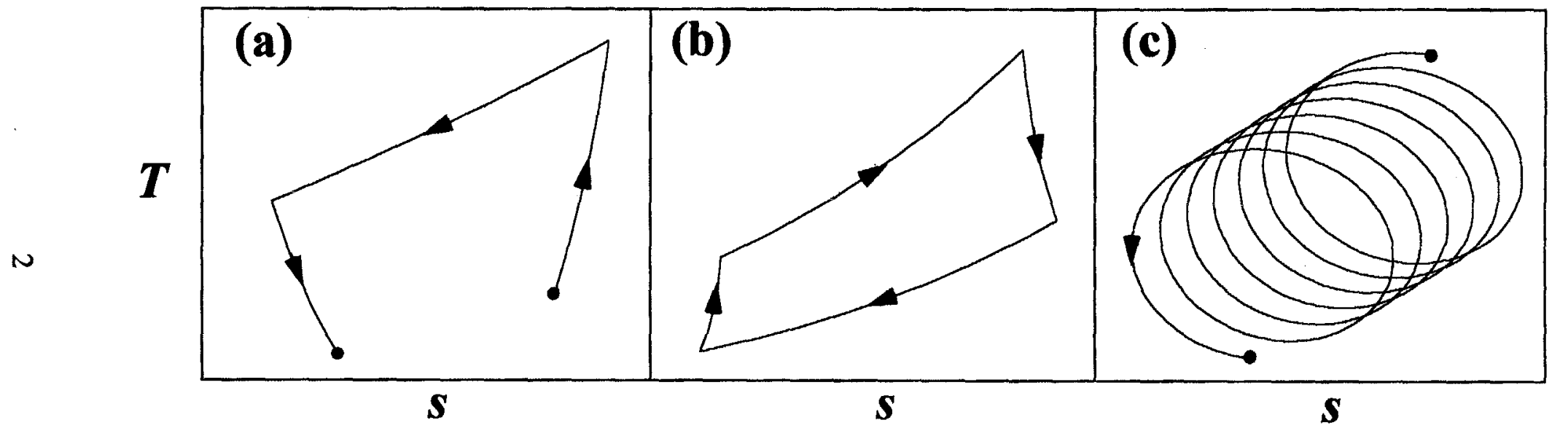

Figure 1. Examples of thermodynamic cycles using a gaseous working fluid. (a) An open thermodynamic process: the Lagrangian path in temperature-entropy space followed by an air-cycle refrigeration system found in jet aircraft. (b) A closed thermodynamic cycle: the Lagrangian $T-s$ path followed by a gas turbine power plant. (c) The superposition of an open process on a closed cycle: the Lagrangian $T-s$ path followed by an open thermoacoustic refrigerator. 
and a process fluid (often air, combustion products, or water). These heat exchangers add weight as well as reduce thermal efficiency by introducing irreversible temperature drops and viscous loss in both fluids. Open thermodynamic systems need fewer heat exchangers since heat transfer can occur directly between the device and the process fluid. The decline of the Stirling engine from widespread use in the $19^{\text {th }}$ century, and the rise of the Diesel and other internal combustion engines, occurred in large part because the latter engines need no combustion-temperature heat exchangers and reject much of their waste heat in their exhaust instead of through ambient-temperature heat exchangers.

When a process fluid must be cooled from a hot ambient temperature $T_{H}$ and delivered to a cold temperature $T_{C}$, these being the same two temperatures that the cyclic thermodynamic refrigerator spans, a substantial efficiency penalty is exacted from irreversible heat transfer between the refrigerator's cold heat exchanger at $T_{C}$ and the incoming process fluid at $T_{H}$. Even for an internally reversible cyclic refrigerator (i.e., having Carnot's performance coefficient) cooling an isobaric ideal-gas stream with mass flow rate $\dot{m}$, the minimum power needed is

$$
\dot{W}_{\min }=\dot{m} c_{p}\left(T_{H}-T_{C}\right)^{2} / T_{C}
$$

where $c_{p}$ is the isobaric heat capacity per unit mass. Equation (1) is the heat removed $\dot{m} c_{p}\left(T_{H}-T_{C}\right)$ times the Carnot performance coefficient $\left(T_{H}-T_{C}\right) / T_{C}$. This minimum 
power is over twice the reversible power required by the First and Second Laws of thermodynamics for this process:

$$
\begin{aligned}
\dot{W}_{r e v} & =\dot{m}\left(h_{C}-h_{H}\right)-\dot{m} T_{H}\left(s_{C}-s_{H}\right) \\
& =\dot{m} c_{p}\left[T_{C}-T_{H}+T_{H} \ln \left(T_{H} / T_{C}\right)\right]
\end{aligned}
$$

where $h$ and $s$ are enthalpy and entropy per unit mass. This result can be viewed as the difference between the incoming and outgoing flow availabilities $h-T_{H} s$.

These shortcomings are simultaneously avoidable. The process fluid itself can be used as the cyclic thermodynamic working fluid, by superimposing its steady flow on the cyclic flow needed for the thermodynamic cycle. Figure 1(c) shows the $T-s$ path followed by this concept.

\section{Description of Closed-cycle Thermoacoustic Devices}

Thermoacoustic devices use acoustic resonators with a high-amplitude sound wave to generate appreciable temperature oscillations in a gas. The sound wave can be generated thermally or mechanically. A coupling occurs between thermal and acoustic energy when gas is allowed to oscillate near, and exchange heat energy with, a solid surface. Thermoacoustic coupling is now being applied in closed thermodynamic cycles to produce power or refrigeration with few moving parts (Swift, 1995). 
Figure 2 depicts a closed-cycle thermoacoustic device. Key components in this device include a gas-filled acoustic resonator, a large-area solid surface called a thermoacoustic stack, and heat exchangers located on either side of the stack. Depending on the magnitude of the stack temperature gradient, this device can act as either a heat engine or refrigerator. The thermoacoustic stack and the heat exchangers consist of a series of parallel plates several solid-thermal-penetration-depths thick; one solid penetration depth is $\delta_{s}=\sqrt{2 K_{s} / \omega \rho_{s} c_{s}}$. The stack and heat exchanger plates are separated from adjacent plates by a distance of several gas-thermal-penetration depths; one gasthermal-penetration depth is $\delta_{\kappa}=\sqrt{2 K / \omega \rho_{0} c_{p}}$.

The sound in a thermoacoustic device exists primarily as a standing wave upon which is superimposed a small traveling-wave component. Locations of minimum amplitude are called nodes, while locations of maximum amplitude are called the antinodes. Near the velocity node of a standing acoustic wave, gas displacement is small. The displacement of the gas increases sinusoidally with distance from the velocity node. In a pure acoustic standing wave the velocity and pressure phases differ by $\pi / 2$. Adiabatic compression of the standing acoustic wave raises the temperature of the gas while displacing it axially toward a pressure anti-node. Adiabatic expansion of a standing acoustic wave reduces the temperature of the gas while displacing it axially away from a pressure anti-node. 

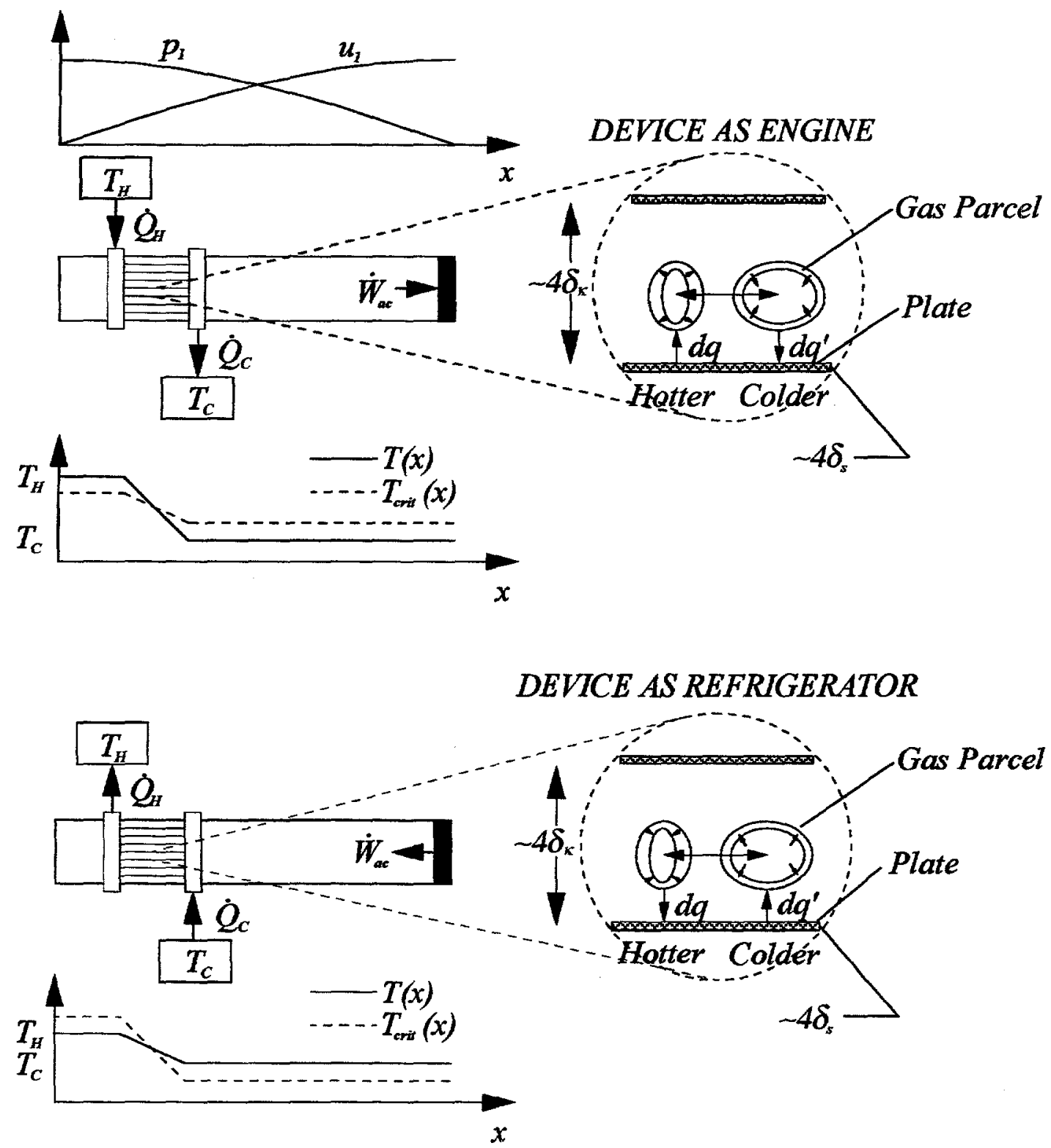

Figure 2. Closed-cycle thermoacoustic devices. 
The stack alters the behavior of the acoustic wave. The temperature of the gas about a gas thermal penetration depth from stack plates tends toward the temperature of the part of the plate from which it just came. Thermoacoustic engines produce work if heat is transferred from the plate to the gas near the phase of greatest compression and heat is transferred from the gas to the plate near the phase of greatest expansion.

Figure 3 shows an idealized thermoacoustic engine cycle for a small gas parcel near a stack plate. An engine cycle begins once an imposed temperature gradient across the stack is large enough for a standing wave to appear in the gas. This cycle can be approximated by four independent idealized thermodynamic processes and heat transfer interactions:

I.-II. The gas parcel absorbs work from its surroundings and is adiabatically compressed as it is displaced toward the velocity node

II.-III. Heat is transferred from the plate to the compressed gas parcel and causes the gas parcel to generate work by transmiting a small traveling wave phase component to the resonator at constant pressure

III.-IV. The gas parcel does work on its surroundings by adiabatically expanding as it is displaced toward the pressure node

IV.-I. Heat is transferred from the expanded parcel to the plate while work is absorbed by the parcel

Similar processes occur to other gas parcels along the length of the stack. It should be emphasized that this four-process cycle is a discrete idealization of the sinusoidally varying path traced between the phase of pressure and density by a real thermoacoustic cycle. These gas parcels collectively produce net acoustic work, which is transmitted to 

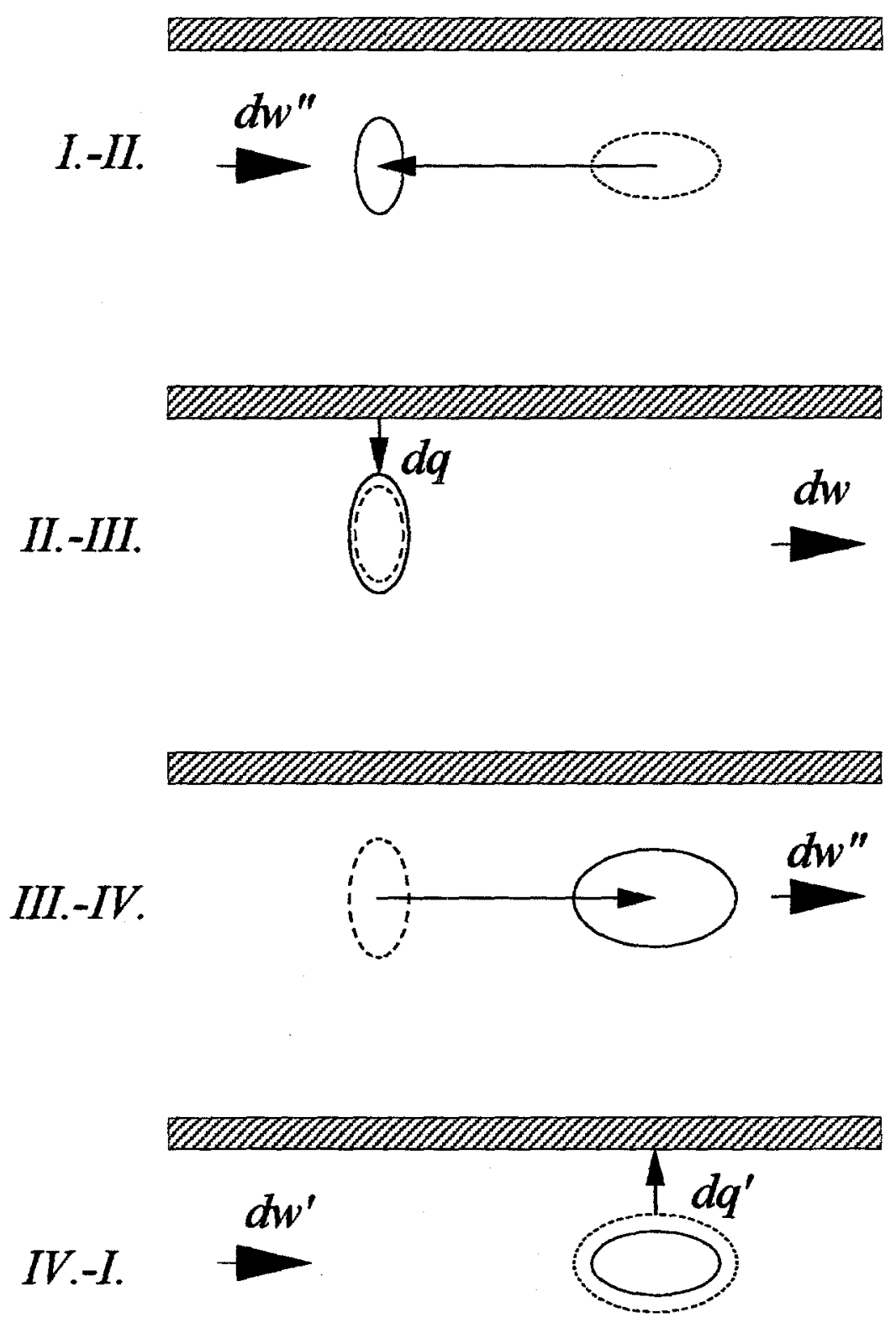

Figure 3. Lagrangian view of the work-heat interaction for a gas parcel near a thermoacoustic stack plate in a simplified thermoacoustic engine cycle. 
the surroundings by a traveling wave. Mechanical or electrical power can be generated when the work from the traveling wave is absorbed by a transducer.

Thermoacoustic refrigerators rely upon similar principles to exchange heat energy between the gas parcel and the stack plate. Thermoacoustic refrigeration occurs if heat is transferred from the working fluid to the plate near the phase of greatest compression and heat is transferred from the plate to the gas parcel near the phase of greatest expansion. Figure 4 shows an idealized thermoacoustic refrigeration cycle for a parcel of gas near a stack plate. This idealized cycle can be divided into four processes:

I.-II. The gas parcel absorbs externally generated work and is adiabatically compressed while being displaced toward the velocity node

II.-III. Heat is transferred from the compressed parcel to the plate while the gas parcel absorbs externally generated work

III.-IV. The gas parcel does work on its surroundings as it adiabatically expands while being displaced toward the pressure node

IV.-I. Heat is transferred from the plate to the expanded parcel while the parcel does work on its surroundings

The net effect of this cycle is a movement of heat along the stack toward a hot reservoir and the velocity node. Similar processes occur to other gas parcels along the length of the stack. These gas parcels collectively produce refrigeration, from thermally or mechanically generated acoustic work. The net effect is to absorb heat from a heat exchanger at the cold end of the stack and to reject it to a heat exchanger at the hot end of the stack. 


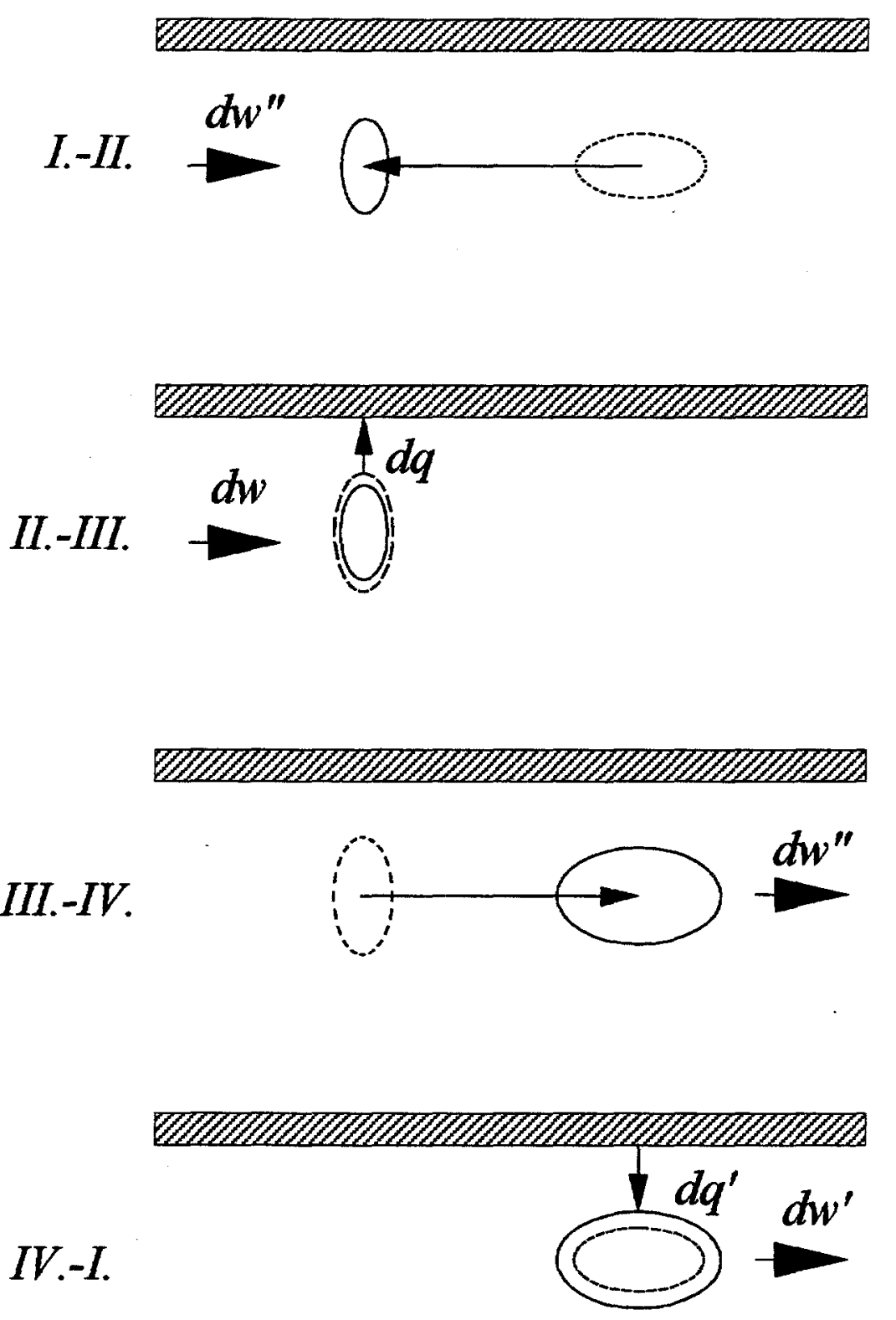

Figure 4. Lagrangian view of the work-heat interaction for a gas parcel near a thermoacoustic stack plate in a simplified thermoacoustic refrigeration cycle. 
The oscillatory flow that is characteristic of a closed thermoacoustic device may be combined with an externally applied mass transfer through the stack - a feature characteristic of an open thermodynamic process. The mass transferred through the thermoacoustic stack can then be heated, cooled, or be made to produce work. With a steady flow of the working fluid through the stack there is a non-zero cyclic oscillatory flow and a non-zero steady flow. The thermal efficiency of inviscid closed-cycle thermoacoustic devices has been well characterized (Swift, 1988). The normalized temperature gradient, $\Gamma$, is the ratio of the stack temperature gradient to the critical temperature gradient and describes the thermodynamic efficiency for inviscid (that is, highly idealized) thermoacoustic devices.

$$
\Gamma=\frac{\nabla T_{0}}{\nabla T_{c r i t}}
$$

If the local temperature gradient along the stack is identical to the temperature gradient imposed on an inviscid gas from adiabatic expansions and contractions without the stack, neither work nor refrigeration is produced. The critical temperature gradient $\Gamma=1$ represents this boundary between the inviscid engine and refrigerator. A stack behaves as a heat engine when the stack temperature gradient is greater than the critical temperature gradient $(\Gamma>1)$. Heat is transferred from the stack to the working fluid during its compressed phase, and rejected to the stack from the working fluid during its expanded 
phase. Thermal efficiency for inviscid closed-cycle thermoacoustic engines is related to Carnot's efficiency by the relation: $\eta=\eta_{\mathrm{c}} / \Gamma$. Note that $\Gamma$ is just the inverse of Second Law effectiveness for inviscid closed-cycle thermoacoustic engines. To prove this: $\dot{W}=\dot{W}_{\text {rev }} / \Gamma$ since $\eta=\dot{W} / \dot{Q}_{H}, \eta_{\mathrm{C}}=\dot{W}_{\text {rev }} / \dot{Q}_{H}$, and $\eta=\eta_{\mathrm{c}} / \Gamma$. The definition of Second Law effectiveness for a heat engine is $\varepsilon=\dot{W} / \dot{W}_{\text {rev }}$, therefore $\varepsilon=1 / \Gamma$. Conversely, if the temperature gradient along the stack is less than the critical temperature gradient $(\Gamma<1)$, the device behaves as a heat pump (or refrigerator). The performance coefficient for closed-cycle thermoacoustic refrigerators with inviscid working fluids is related to Carnot's performance coefficient by the relation: $\boldsymbol{\beta}_{1}=\Gamma \cdot \boldsymbol{\beta}_{1, \mathrm{C}}$.

\section{Background}

Until recently, devices that couple thermal and acoustic energy have been used in few practical applications (Rott, 1983). A rudimentary form of a thermoacoustic engine was demonstrated by Byron Higgins in 1777. Higgins produced acoustic oscillations inside a large cylindrical tube with a hydrogen flame placed inside it. These oscillations resonate at the fundamental acoustic mode or one of the harmonics of the tube.

Devices resembling the proposed open-cycle thermoacoustic refrigerator are even more rare. In the $19^{\text {th }}$ century, Rijke noticed that a heated metal gauze located at about one quarter of the distance from the bottom of an open vertical tube results in acoustic excitation (Feldman, 1968). Rijke explained that the air current that flows through the 
tube expands near the heated screen and compresses downstream from the screen due to the cooling of the gas by the tube walls. A related effect was discovered by Bosscha and also by Reiss who showed that a cooling element placed three quarters of the distance from the bottom of an open vertical tube also results in acoustic excitation. Rayleigh (1945) wrote that the excitations observed by Rijke, Bosscha, and Reiss consist of compression and expansion oscillations superimposed on a steady upward convection current of air. Heat is transferred to the air at the moment of greatest compression, while heat is rejected from the air at the moment of greatest rarefaction. This explanation is often referred to as the Rayleigh criterion (Feldman, 1968).

Close antecedents to the concept introduced in this work are believed to be pulse combustion, as applied to the V-1 buzz bomb of the Second World War and to modern residential gas furnaces (Zinn, 1986), and the Boreas cryocooler (Crunkleton, 1988). However, the pulse combustor does not use its oscillatory pressure in any thermodynamic sense (only to pump air through the burner using check valves) and the Boreas cooler does not use its superposition of steady and oscillatory flows to eliminate heat exchangers.

Rijke's tube and the proposed concept for open thermoacoustic devices share some common features. Both Rijke's tube and open thermoacoustic devices exploit a conversion between thermal and acoustic energy. In contrast, however, a Rijke tube is an acoustic heat engine with a steady flow produced by buoyancy forces, while the proposed open thermoacoustic device is a refrigerator with an externally applied flow. 
Two well-known thermoacoustic effects in partially closed resonators are the Sondhauss and the Taconis oscillations (Swift, 1988). Sondhauss oscillations occur when heat is applied to the closed end of a gas-filled resonator. Taconis oscillations occur when a temperature gradient between cryogenic temperature and room temperature spans part of the length of a resonator that is closed on the room temperature end. The Rayleigh criterion provides a qualitative explanation of Sondhauss oscillations and (with some extension) of Taconis oscillations, but a satisfactory quantitative explanation of these oscillations remained elusive until the late 1960's. Rott (1969) published the first of a series of papers that considered these oscillations in long tubes with diameters both large and small when compared to the thermal penetration depth of the oscillating medium. This work eventually led to a general quantitative theory of linear heat-driven oscillations (Rott, 1975) near solid surfaces where viscous forces dominate and velocity and force are in phase. Rott's theory has been since confirmed in numerous experiments, starting with Yazaki et al. (1979).

Recently, Rott's theory has been applied to thermoacoustic engines, heat pumps, and refrigerators at Los Alamos National Laboratory (Swift, 1992) and the Naval Postgraduate School in Monterey, California (Garrett, 1992). A number of refrigerators and heat engines based on the thermoacoustic operating cycle have been produced (Hofler, 1988). Rott's theory has been successfully used to predict the performance of these devices when they operate at low amplitude (Ward, 1994). Rott's theory was recently applied to experimental data taken with an open-cycle thermoacoustic 
refrigerator (Reid, Ward, and Swift, 1998). Data from that article are more comprehensively reported in this dissertation along with additional results. 


\section{CHAPTER 2}

\section{SOME FEATURES OF OPEN CYCLE THERMOACOUSTICS}

Several simple examples serve to illustrate possible open-cycle thermoacoustic devices. These examples are intended to put the refrigerator described in Chapter 4 into context. For convenience, inviscid working-fluid behavior is assumed in the first part of this discussion. Figure 5 shows four half-wavelength open-cycle thermoacoustic devices. Common to each device is a resonator containing a gas that supports an acoustic standing wave. Velocity antinodes are located at either end of each resonator. Also, common to these devices are heat exhangers, a stack, an applied flow source, and an electromechanical transducer capable of either supplying acoustic power to or extracting acoustic power from the device.

The stack in Device (A) absorbs acoustic power, $\dot{W}_{a c}$, supplied by the electromechanical transducer. The power absorbed in the stack causes enthalpy to be removed at a rate $\dot{Q}_{H}$ from the gas stream flowing toward the stack cold end as well as 
(A)

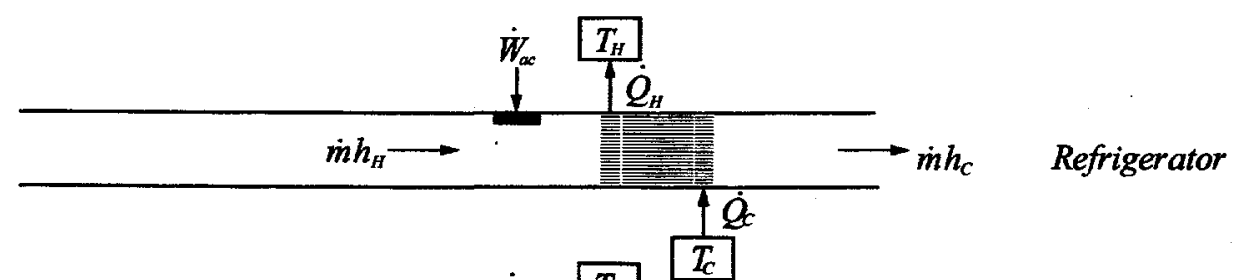

(B)

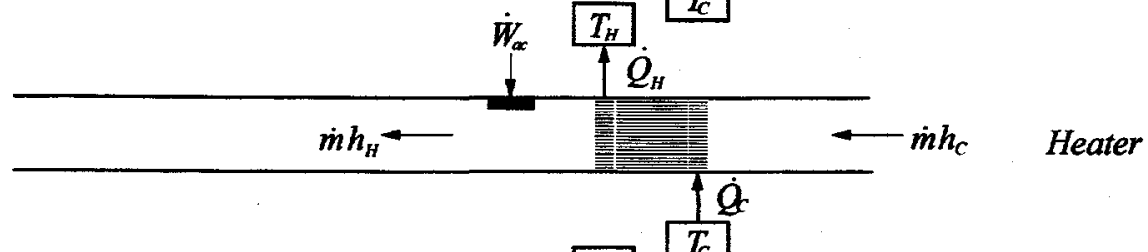

(C)

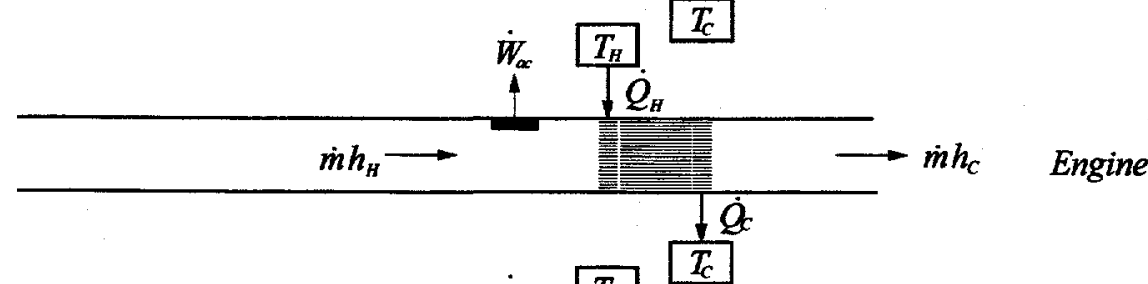

(D)
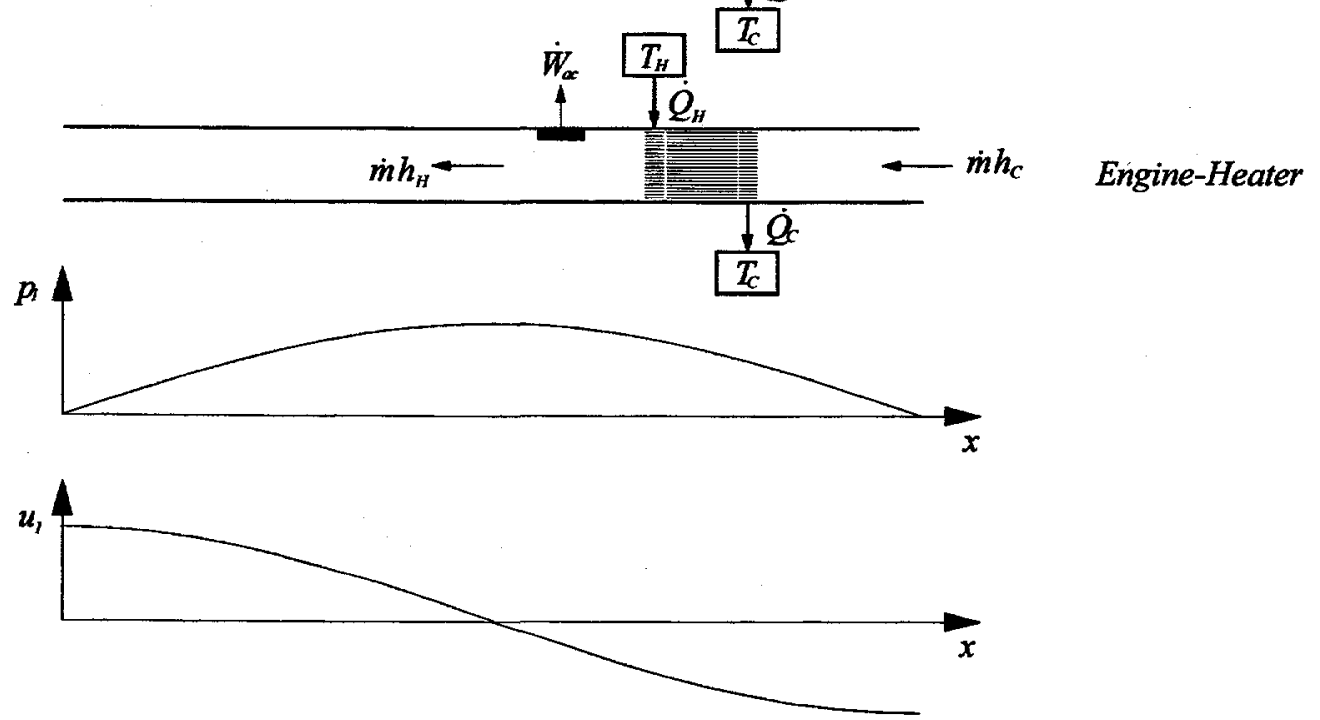

Figure 5. Comparison of four different half-wavelength open-cycle thermoacoustic devices. 
any heat at a rate $\dot{Q}_{C}$, absorbed by the stack at the cold reservoir. Energy conservation requires that the heat rejection rate, $\dot{Q}_{H}$, is equal to $\dot{W}_{\text {ac }}$, plus the enthalpy flow rate difference between the entering gas stream, $\dot{m} h_{H}$, and exiting gas stream, $\dot{m} h_{C}$ and any absorbed thermal energy rate, $\dot{Q}_{c}$.

Reversing the flow direction produces the situation represented by Device (B). Here, the gas stream is heated as it moves toward the hot end of the stack. Without applied flow, both devices are identical and can behave as a heat pump or a refrigerator. (The terms "refrigerator" and "heat pump" customarily refer to work absorbing devices that transfer heat from a cold to a hot reservoir. "Refrigerator" is used when the desired effect is thermal energy removal from a cold reservoir. "Heat Pump" is used when thermal energy rejection to a hot reservoir is desired.) The introduction of applied flow allows a number of distinct manners of use. Among these are:

1. Device (A) operates as a refrigerator with respect to the thermal energy reservoirs while cooling the applied flow that moves through the stack.

2. Device (A) operates in mixed mode, heat pumping with respect to the thermal energy reservoirs while cooling the applied flow that moves through the stack.

3. Device (B) operates in mixed mode, refrigerating with respect to the thermal energy reservoirs while heating the applied flow that moves through the stack.

4. Device (B) operates as a heat pump with respect to the thermal energy reservoirs while heating the applied flow that moves through the stack. 
To simplify discussion, Device (A) will be called an "open thermoacoustic refrigerator" since its net effect is to cool a gas stream passing though the stack. Conversely, "open thermoacoustic heater" will be used to describe Device (B) since it heats the gas stream flowing through it. This simplification is made with the understanding that mixed use of either device is possible.

The mean temperature gradient, $\nabla T_{0}$, along the stack of the refrigerator and heater is less than the critical temperature gradient, $\nabla T_{c r i t}$. If the mean temperature gradient is increased to significantly exceed the critical temperature gradient, the thermoacoustic device can operate as a heat engine. The desired effect of Device (C) is to produce mechanical work at a rate, $\dot{W}_{\mathrm{ac}}$, while a stream of gas flows toward the cold end of the stack. Part of $\dot{Q}_{H}$, supplied at temperature, $T_{H}$, and enthalpy from the entering applied flow, $\dot{m} h_{H}$, generate an acoustic wave with standing and traveling components in pressure and velocity. The traveling wave transmits acoustic power that is absorbed by the transducer. The part of $\dot{Q}_{H}$ not used to produce work is either rejected to the cold reservoir, $\dot{Q}_{C}$, or is retained by the flow leaving the cold heat exchanger, $\dot{m} h_{C}$. Energy conservation requires that the power generated by the device be equal to the heat supplied at the hot reservoir at a rate, $\dot{Q}_{H}$, the heat rejected to the cold reservoir at a rate, $\dot{Q}_{C}$, and the enthalpy difference between the entering and exiting streams.

Device (D) also produces mechanical work at a rate, $\dot{W}_{\text {ac }}$, but heats a stream of gas flowing toward the hot heat exchanger. Devices (C) and (D) are named in a manner 
analogous to the refrigerator and heater previously discussed. Device (C) will be called an "open thermoacoustic engine" since power is produced and the thermoacoustic and applied enthalpy flows in it are from hot to cold. Device (D) will be called an "open thermoacoustic engine-heater" since the applied flow is heated while work is produced. As with the refrigerator and heater, the introduction of applied flow through an engine stack allows several additional manners of use:

1. Device (C) produces net power from hot reservoir-supplied heat and the change in enthalpy of the applied flow.

2. Device (C) operates in mixed mode, producing net power at the expense of energy rejection to the device surroundings.

3. Device (D) operates in mixed mode, producing net power while heating the applied flow that moves through the stack at the expense of heat rejection to the cold reservoir.

4. Device (D) produces net power while heating the applied flow that moves through the stack from heat supplied at the hot reservoir.

Open thermoacoustic devices have several interesting and potentially useful features. First, they need fewer heat exchangers than the equivalent closed-cycle thermoacoustic engine and refrigerator cycles shown in Chapter 1. The cold heat exchanger inside the refrigerator and heater and the hot heat exchanger inside the engine and engine-heater are completely unnecessary for open thermoacoustic operation. External heat exchangers that transfer heat between these internal heat exchangers and the thermal energy reservoirs are also eliminated, reducing irreversibility from viscous loss and temperature drops. 
Second, the working fluid in closed-cycle thermoacoustic devices, typically a highsound-speed inert gas such as helium, must be sealed in pressure vessels. Open thermoacoustic devices may require only modest structure since they can be designed to operate near ambient pressure. However, operation near ambient pressure has a significant drawback: acoustic power density is proportional to the square of the acoustic pressure amplitude. Open devices operating at low pressure would require a large stack surface area to achieve power densities comparable to closed-cycle thermoacoustic devices that typically operate at mean pressures of ten atmospheres or more.

Many additional combinations are suggested by this discussion. The temperatures of the entering and exiting streams might differ from their respective reservoir temperatures. An open thermoacoustic refrigerator driven by a thermoacoustic engine might be made without moving parts to make it more reliable than a mechanically driven one. Limited time and resources necessitate the careful selection of a first open-cycle thermoacoustic prototype. Choices such as thermally driven refrigerators are not suited to a well-controlled experimental demonstration since the measurement of acoustic power produced by a thermally driven acoustic engine is difficult. The demonstration of an easily characterized but inefficient mechanically driven prototype that can operate as a refrigerator or a heater has been selected for this work. The demonstration of the engine and engine-heater devices awaits the work of others. 


\section{Nondimensional Forms of the Governing Equations}

In this section, a set of variables and relations that describe the state and linear motion of a thermoacoustic working fluid over a stack plate are introduced. Proposed continuity, momentum, and energy equations given in Appendix A will be summarized along with the underlying assumptions used to derive them. The variables in these equations will be cast in nondimensional form by identifying the major scales of the physical quantities. These scales will then be used to derive scales for the remaining variables. The governing equation will then be normalized to obtain the similitude parameters. Thermoacoustic devices exploit several natural scaling relations between the resonator and stack geometry and the thermophysical properties of the materials that comprise it. The resonator length most conveniently scales to the fundamental resonant wavelength of the working fluid. Generally, the stack should be much longer than the gas displacement amplitude. The spacing between thermoacoustic stack plates scales to the thermal and viscous penetration depths of the working fluid. The thermoacoustic stack plate thickness for high-power-density stacks relates directly to the thermal penetration depth of the plate material. Finally, working fluid pressure and sound speed, along with the stack plate density and the radius of the resonator scale to the total acoustic power.

Consider a stack consisting of a series of rigid, solid, impermeable, parallel plates with length $L$ in the $x$ direction. The $y$ direction is normal to the plate surfaces. Each plate is $2 \delta_{s}$ thick and separated from its neighbors by gas-filled channels with thickness $2 y_{0}$, where $y_{0} \sim \delta_{v}$ and $y_{0} \sim \delta_{k}$. The values $\delta_{s}$ and $\delta_{k}$ are the lengths heat diffuses in 
time $1 / \omega$ in the solid and gas, respectively. Similarly, the value $\delta_{v}$ is the momentum diffusion distance in the gas over time $1 / \omega$. For most gases of interest, $\delta_{\kappa} \sim \delta_{v}$ applies. The coordinate system is defined so that $y=0$ is the center of the gas-filled channel, and $\pm y_{0}$ are the plate surfaces bounding each channel. The channel width in the $z$ direction is arbitrary (but in any case is much greater than $y_{0}$ ). At $x=0$ and $x=L$ the gas-filled channel above the plate edges is open. The gas-filled region is sealed and impermeable along the plate edges on the $\pm z$ sides.

An externally generated standing sound wave with frequency $f$ is produced in the gas-filled region along the $x$ direction with a radian wavelength much greater than the stack length $L$. Since periodic changes occur over a standing wave cycle, the inverse of the resonance frequency serves as a convenient scale for time-varying quantities. At a point upstream of the stack is a region not affected by the presence of the stack plates. This region is characterized by the steady applied flow terms $U=U_{\infty}, V=0, p=p_{\text {ref }}$, and $T=T_{\text {ref }}$. An externally applied flow of gas enters the stack at $x=0$ and moves toward $x=L$. In the space between plates, the relation among the stack plate thickness, $l$, the free stream applied flow velocity, and the stack-entrance applied flow velocity is:

$$
u_{a}=u_{\infty} \frac{l+y_{0}}{y_{0}}
$$


This applied flow is superimposed on the oscillatory flow of the standing wave with its velocity component parallel to the oscillatory flow.

The stack inlet (the reference temperature) is taken to be on the hot heat exchanger side when the device operates as a refrigerator and on the cold heat exchanger side when the device operates as a heater. The acoustic power supplied by the driver and the volumetric velocity of the applied flow are treated independent variables. The dependent variables and their domains used here are

$$
\begin{gathered}
\rho=\rho_{0}(x)+\rho_{0}^{\prime}(y, z)+\operatorname{Re}\left[\rho_{1}(x, y, z) e^{i \omega x}\right] \\
u=u_{a}(x, y, z)+\operatorname{Re}\left[u_{1}(x, y, z) e^{i \omega x}\right] \\
v=v_{a}(x, y, z)+\operatorname{Re}\left[v_{1}(x, y, z) e^{i \omega x}\right] \\
w=w_{a}(x, y, z)+\operatorname{Re}\left[w_{1}(x, y, z) e^{i \omega x}\right]
\end{gathered}
$$

The relation for density found in Equation (5) applies for the other thermophysical properties as well. Thermodynamic and thermophysical properties that appear in dimensionless form are evaluated at the reference temperature and pressure. On the left side of the equations are real variables. The oscillatory velocity and density quantities inside the real operators are also complex. The mean density and the mean density variation in the transverse directions are both real variables. The first terms on the right 
side of the three velocity equations are real quantities representing the applied flow components of velocity.

\section{Mass Continuity}

The equation of continuity for a homogenous compressible substance is

$$
\frac{\partial \rho}{\partial t}+\nabla \cdot(\rho \mathbf{v})=0
$$

The oscillatory components of density and velocity that are proportional to $e^{i \omega t}$ and the time-independent applied flow components of density and velocity in the continuity equation can be separated. Oscillatory terms smaller than first order and applied flow terms smaller than second order can be eliminated.

The velocity of the applied flow upstream of the stack entrance is assumed to be steady, uniform, and one-dimensional in the $x$ direction. Consider a region positioned near the middle of the stack at $L / 2$ such that $\delta_{v} \ll L / 2$. The channel dimensions make the axial velocity terms much larger than either transverse component. In the mid-stack region the applied flow part of the continuity equation can be taken to be fully developed. In practice, fully developed steady flow is justified over all but a short distance near the stack entrance, $\ell_{e} \ll L$. Applied volumetric velocity can change slightly due to the dependence of the mean temperature and mean pressure on axial position. The mean 
pressure $p_{0}$ varies with axial position due to viscous pressure drop, but for the flow velocities and gas region dimensions considered here, this change can be ignored. Combining Equations (5) through (9), separating the non-oscillatory from the oscillatory components, and eliminating unsteady terms and steady terms smaller than second order, the mass conservation equation for fully developed applied flow through the stack is

$$
\frac{d}{d x} \rho_{0} U_{a}=0
$$

The inclusion of density in the derivative term reflects the changes in density with mean temperature that can occur along the stack. Each of the variables in this equation is real. The axial applied volumetric flow rate is an integrated value across the transverse plane

$$
U_{a}=\int_{A_{\text {flud }}} u_{a} d y d z=A_{\text {fluid }}\left\langle u_{a}\right\rangle
$$

The steady applied flow in the stack region then expands or contracts in response to mean temperature changes with corresponding increases or decreases in the applied volumetric flow rate.

Again, consider the region near the middle of the stack at $L / 2$ such that $\delta_{v} \ll L / 2$. Since both the stack plates and the $\pm z$ walls are impermeable, the $z$-direction oscillatory velocity component is of negligible magnitude. The $v_{1}(y)$ component contributes some to 
$\rho_{1}(y)$. A no-slip boundary is assumed to exist for axial oscillatory velocity at the surface of the stack plate so $u_{1}\left(y_{0}\right)=0$. If the oscillatory velocity and density are integrated across the gas-filled region, the continuity equation for the oscillatory and applied flows can be reduced to:

$$
\omega A_{\text {fluid }} \operatorname{Re}\left[\left\langle\rho_{1}\right\rangle i e^{i \omega t}\right]+\frac{d}{d x} \rho_{0} \operatorname{Re}\left[U_{1} e^{i \omega x}\right]=0
$$

The spatially averaged density, $\left\langle\rho_{1}\right\rangle$, oscillates about a local value of $\rho_{0}$ and leads the gradient of oscillatory component of mass flow rate by $\pi / 2$. Combining the previously separated steady and oscillatory component equations, using the ideal gas state equation, and normalizing each variable using the identified scales $f, T_{\text {ref }}, p_{\text {ref }}$, and $L$ yields the dimensionless continuity relation in the gas-filled region between stack plates

$$
2 \pi \operatorname{Re}\left[\left\langle\bar{p}_{1}-\bar{T}_{1}\right\rangle i e^{i 2 \pi \tilde{f}}\right]+\frac{d}{d \bar{x}}\left(\left\langle\xi_{a}\right\rangle+\operatorname{Re}\left[\left\langle\xi_{1}\right\rangle e^{i 2 \pi \bar{f}}\right]\right)+\left(\left\langle\xi_{a}\right\rangle+\operatorname{Re}\left[\left\langle\xi_{1}\right\rangle e^{i 2 \pi \bar{f}}\right]\right) \frac{\bar{T}_{0}}{\bar{p}_{0}} \frac{d}{d \bar{x}}\left(\frac{\bar{p}_{0}}{\bar{T}_{0}}\right)=0
$$

The variables with a bar above them are dimensionless quantities. Definitions of these and dimensionless variables introduced later are listed in Table 1. 
Table 1. List of Some Dimensionless Variables

\begin{tabular}{|c|c|c|c|c|}
\hline Variable & $\begin{array}{l}\text { Type and } \\
\text { Domain }\end{array}$ & Symbol & Definition & Interpretation \\
\hline Operating Frequency & $\begin{array}{l}\text { Independent } \\
\text { Global }\end{array}$ & $\overline{\bar{f}}$ & $f / f_{\text {ref }}$ & Ratio of operating frequency to reference frequency (normally unity) \\
\hline Mean Pressure & $\begin{array}{l}\text { Dependent } \\
\text { Global }\end{array}$ & $\bar{p}_{0}$ & $p_{0} / p_{\text {ref }}$ & Dimensionless mean pressure of working fluid (normally unity) \\
\hline Dynamic Pressure & $\begin{array}{l}\text { Independent } \\
\text { Global }\end{array}$ & $\bar{p}_{1}$ & $p_{1}(x, t) / p_{\text {ref }}$ & Dimensionless time and spatial dependent dynamic pressure of working fluid \\
\hline Time & $\begin{array}{l}\text { Independent } \\
\text { Global }\end{array}$ & $\bar{t}$ & tfef & Number of cycles \\
\hline Mean Temperature & $\begin{array}{l}\text { Dependent } \\
\text { Stack }\end{array}$ & $\bar{T}_{0}$ & $T_{0}(x) / T_{\text {ref }}$ & Dimensionless spatially dependent working fluid temperature \\
\hline Dynamic Temperature & $\begin{array}{l}\text { Dependent } \\
\text { Stack }\end{array}$ & $\bar{T}_{1}$ & $T_{1}(x) / T_{\text {ref }}$ & Dimensionless dynamic working fluid temperature \\
\hline Position & $\begin{array}{l}\text { Dependent } \\
\text { Stack }\end{array}$ & $\bar{x}$ & $x / L$ & Ratio of position along stack to total stack length \\
\hline Position & $\begin{array}{l}\text { Dependent } \\
\text { Stack }\end{array}$ & $\bar{y}$ & $y / y_{0}$ & Ratio of position normal to stack surface to plate separation distance \\
\hline Ratio of Specific Heats & $\begin{array}{l}\text { Independent } \\
\text { Global }\end{array}$ & $\gamma$ & $c_{p} / c_{p}$ & Ratio of isobaric to isochoric specific heats \\
\hline Womersley Number & $\begin{array}{l}\text { Independent } \\
\text { Stack }\end{array}$ & Wo & $y_{0} / \delta_{v}$ & Ratio of oscillatory inertial to viscous forces \\
\hline Applied Flow Velocity & $\begin{array}{l}\text { Independent } \\
\text { Stack }\end{array}$ & $\xi_{a}$ & $u_{a} / f_{\text {ref }} L$ & Ratio of acoustic period to transit time for a gas particle to traverse the stack \\
\hline Oscillatory Flow Velocity & $\begin{array}{l}\text { Independent } \\
\text { Stack }\end{array}$ & $\xi_{1}$ & $u_{1} / f_{\text {ref }} L$ & Ratio of oscillatory particle displacement length to total stack length \\
\hline Enthalpy Flux Ratio & $\begin{array}{l}\text { Dependent } \\
\text { Stack }\end{array}$ & $\mathbf{\Xi}$ & $\Delta \dot{H}_{0} / \dot{H}_{2}$ & Ratio of the sensible heat flux extracted from applied flow to second order heat flux \\
\hline Prandtl Number & $\begin{array}{l}\text { Independent } \\
\text { Global }\end{array}$ & $\sigma_{\text {ref }}$ & $\mu_{\text {ref }} c_{p} / K_{\text {ref }}$ & $\begin{array}{l}\text { Ratio of the molecular momentum diffusivity to thermal diffusivity of the working } \\
\text { fluid at the reference condition }\end{array}$ \\
\hline
\end{tabular}


The dimensionless applied volumetric flow rate $\left\langle\xi_{a}\right\rangle=U_{a} / f L A_{\text {fluid }}$ is a real quantity. One possible interpretation for $\left\langle\xi_{a}\right\rangle$ is the ratio of the acoustic period, $\tau=1 / f$, to the mean transit time $\tau_{t}=L A_{\text {fluid }} / U_{a}$ for a gas particle to move across the stack. Another interpretation for this quantity is the mean fractional stack length displaced by a gas particle during a standing wave cycle. For the flow conditions that will be considered in this work, the limits of this parameter are $-2.46 \cdot 10^{-2}<\left\langle\xi_{a}\right\rangle<2.56 \cdot 10^{-2}$. The other group $\left\langle\xi_{1}\right\rangle=U_{1} / f L A_{\text {fluid }}$ is a complex dimensionless quantity whose magnitude is equal to the oscillatory particle displacement amplitude divided by the stack length. The magnitude of this quantity, for the conditions considered here, is typically 0.29 at the entrance of the stack and 0.31 at the stack exit.

\section{Momentum Equation}

Neglecting the second (bulk) viscosity, the momentum equation for a general Newtonian fluid given in Landau (1987) and found in Appendix A is

$$
\rho\left[\frac{\partial \mathrm{v}}{\partial t}+(\mathbf{v} \cdot \nabla) \mathbf{v}\right]=-\nabla p+\frac{\partial}{\partial \mathrm{x}_{k}}\left[\mu\left(\frac{\partial \mathrm{v}_{i}}{\partial \mathrm{x}_{k}}+\frac{\partial \mathrm{v}_{k}}{\partial \mathrm{x}_{i}}-\frac{2}{3} \delta_{i k} \frac{\partial \mathrm{v}_{l}}{\partial \mathrm{x}_{l}}\right)\right]
$$

Focusing on the velocity component in the axial direction, assuming constant mean thermophysical properties (implying $\Delta T_{0} \ll T_{0}$ ), and assuming the axial viscous 
derivatives are small compared to the transverse viscous derivatives, the oscillatory momentum equation reduces to

$$
\omega \rho_{0} \operatorname{Re}\left[u_{1} i e^{i \omega t}\right]=-\frac{d}{d x} \operatorname{Re}\left[p_{1} e^{i \omega t}\right]+\mu \frac{d^{2}}{d y^{2}} \operatorname{Re}\left[u_{1} e^{i \omega x}\right]
$$

The dimensionless form of the oscillatory momentum equation is

$$
2 \pi \operatorname{Re}\left[\xi_{1} i e^{i 2 \pi \bar{f}}\right]=-\frac{1}{\bar{y}} \frac{d}{d \bar{x}} \operatorname{Re}\left[\bar{p}_{1} e^{i 2 \pi \bar{f}}\right]+\frac{\delta_{v}^{2}}{y_{o}^{2}} \frac{d^{2}}{d \bar{y}^{2}} \operatorname{Re}\left[\xi_{1} e^{i 2 \pi \bar{f}}\right]
$$

This equation expresses a balance between the driving longitudinal pressure gradient, the inertia of the gas, and the viscous friction posed by the stack plate on the adjacent gas. Near the plate the viscous term dominates. Farther away from the plate viscous forces become small and inertia begins to dominate. For the spacing between stack plates considered here the viscous term is more important than the inertia term. The coefficient of the pressure gradient term is the inverse of the product of specific heat ratio and dimensionless frequency. The coefficient of the viscous term is the square of the viscous penetration depth, $\delta_{v}=\sqrt{v / \pi f}$, over the half-distance between stack plates, $y_{0}$. This coefficient is the inverse of the Womersley number squared for oscillating flow where the length scale is $y_{0}$, the oscillating inertial force is contained in frequency, $f$, and the 
viscous force is contained in the kinematic viscosity $v$. For the conditions in the stack, this parameter is of order unity. The parameter leading the pressure term in Equation (16) is of order 10 for a system consisting of an ideal monotonic gas.

Using arguments similar to those used to derive the continuity equation, the applied flow momentum equation in the stack can be separated from the oscillatory equation.

$$
0=-\frac{d p_{0}}{d x}+\mu \frac{d^{2} u_{a}}{d y^{2}}
$$

This equation expresses the relation between the driving force imposed by the pressure gradient and the dissipative viscous forces. For the applied flow velocities considered here the magnitude of these terms are small. Again, the Womersley number for applied flow through the stack is of order unity. Assuming then that the flow through the stack is laminar, the dimensionless applied flow momentum equation for an ideal gas is

$$
0=-\frac{1}{\bar{f}} \frac{d \bar{p}_{0}}{d \bar{x}}+\frac{\delta_{v}^{2}}{y_{o}^{2}} \frac{d^{2} \xi_{a}}{d \bar{y}^{2}}
$$

the parameters on the right side of this equation are identical to those found in Equation (16). Like the oscillating momentum equation, the applied flow momentum equation is valid for all but a tiny fraction of the stack length. 


\section{Energy Equation}

Ignoring the second (bulk) viscosity, the thermal energy equation for a Newtonian fluid given in Landau (1987) and found in Appendix A is

$$
\rho T\left[\frac{\partial s}{\partial t}+(\mathbf{v} \cdot \nabla) s\right]=\nabla \cdot(K \nabla T)+\frac{1}{2} \mu\left(\frac{\partial \mathrm{v}_{i}}{\partial \mathrm{x}_{k}}+\frac{\partial \mathrm{v}_{k}}{\partial \mathrm{x}_{i}}-\frac{2}{3} \delta_{i k} \frac{\partial \mathrm{v}_{l}}{\partial \mathrm{x}_{l}}\right)^{2}
$$

Within a thermoacoustic stack, the dependence of mean temperature on axial position is changed by a non-zero applied volumetric flow velocity. This dependence can be separated into applied and oscillatory components in the energy equation. Substituting $d s=\left(c_{p} / T\right) d T-(\beta / \rho) d p$ and keeping only first order terms, the simplified dimensionless oscillatory energy equation in the stack is given by

$$
2 \pi \bar{f} \operatorname{Re}\left[\bar{T}_{1} i e^{i 2 \pi \bar{f}}\right]+\operatorname{Re}\left[\xi_{1} e^{i 2 \lambda \bar{f}}\right] \frac{d \bar{T}_{0}}{d \bar{x}}-2 \pi \bar{f} \frac{\gamma-1}{\gamma} \cdot \operatorname{Re}\left[\bar{p}_{1} i e^{i 2 \pi \bar{f}}\right]=\frac{\delta_{v}^{2}}{y_{o}^{2}} \frac{1}{\sigma_{r e f}} \frac{d^{2}}{d \bar{y}^{2}} \operatorname{Re}\left[\bar{T}_{1} e^{i \bar{\omega} t}\right](20)
$$

This form of the energy equation contains the usual inverse Peclet number term (with Womersley number squared as Reynolds number surrogate). For an ideal gas this term is of order unity. The other terms are dimensionless angular velocity of order 10 and a group containing the ratio of specific heats of order unity. The simplified dimensionless energy equation for applied laminar flow of an ideal gas through the stack is 


$$
\xi_{a} \frac{d \bar{T}_{0}}{d \bar{x}}=\frac{\delta_{v}^{2}}{y_{o}^{2}} \frac{1}{\sigma_{r e f}} \frac{d^{2} \bar{T}_{0}^{\prime}}{d \bar{y}^{2}}
$$

the form of Equation (21) varies only slightly from that of Equation (20). The Prandtl number for the gas mixture considered in Chapter 4 is about 0.5 .

\section{Inviscid Short Stack Approximation}

The general results based on Rott's equation mentioned in Chapter 1 and presented in Appendix A yield accurate solutions, but require lengthy integration. A simplified, inviscid description of the effect of applied flow on the temperature distribution along a thermoacoustic-refrigerator stack plate is derived. This description makes use of the zero viscosity assumptions described in Swift (1988). The result derived here is then generalized to describe devices other than refrigerators.

Consider a quarter-wavelength standing wave defined between $0<X<\lambda / 4$. For a refrigerator, the applied flow moves with increasing $X$ toward the pressure node located at $X=\lambda / 4$. At some reference position, $X=X_{\text {ref }}$, far from the pressure and velocity nodes, is a stack plate with domain $0<x<L$. To lowest order approximation, all points along this plate are assumed to have the same thermophysical properties, oscillatory pressure, and oscillatory velocity as exists at the single position $X_{\text {ref }}$ in the standing wave. This assumption is reasonable since $\lambda / 4$ is typically much longer than the stack 
length, $L$. The stack end located at $x=0$ has temperature $T_{H}$. The opposite end of the stack has temperature $T_{C}$ and location $x=+L$. For a refrigerator configured like this, the applied flow moves along the $x$-axis toward $x=+L$. The coordinate systems are related by $x=X-X_{\text {ref }}+L / 2$.

For a thermoacoustic refrigerator with no applied flow, the oscillatory pressure wave of the standing wave can be described by $p_{1}=p_{1}^{s} e^{i(0)}$ where the positive-real variable $p_{1}^{s} \equiv p_{A} \cos \left(X_{r e f} / \lambda\right)$ along the stack. Using the one-dimensional momentum equation, $i \omega \rho_{0} u_{1}=-d p_{1} / d X$, the $X$-component of oscillatory velocity can be found: $u_{1}=\left(p_{A} / \rho_{0} a\right) \sin \left(X_{r e f} / \lambda\right) e^{i(-\pi / 2)}$ where $u_{1}^{s} \equiv\left(p_{A} / \rho_{0} a\right) \sin \left(X_{r e f} / \lambda\right)$ at all points along the stack. Here it is assumed that the stack thickness is much less than the spacing between stack plates: $l \ll y_{0}$.

The equation for the second-order enthalpy flux along the stack in terms of $p_{1}, U_{1}$, $T_{0}$, material properties, and geometry given in Appendix A and (in abbreviated form) in Reid, Ward, and Swift (1998) is

$$
\begin{aligned}
\dot{H}_{\text {tot }} & =\dot{H}_{2}=\frac{T_{0} \beta}{2} \operatorname{Re}\left[p_{1} \tilde{U}_{1}\left(1-\frac{\left(f_{K}-\tilde{f}_{v}\right)}{(1+\sigma)\left(1-\tilde{f}_{v}\right)}\right)\right] \\
& +\frac{\rho_{0} c_{p}\left|U_{1}\right|^{2}}{2 \omega A_{\text {fuid }}\left(1-\sigma^{2}\right)\left|1-f_{v}\right|^{2}} \operatorname{Im}\left[f_{\mathrm{K}}+\tilde{\sigma}_{v}\right] \frac{d T_{0}}{d x} \\
& -\left(A_{\text {fuid }} K+A_{\text {solid }} K_{\text {solid }}\right) \frac{d T_{0}}{d x}
\end{aligned}
$$


If the working fluid is assumed to be inviscid, $\sigma=0$ and the complex function $f_{v}=0$. The complex function $f_{x}$ takes on the values 0 and 1 in the isentropic and isothermal limits, respectively, so $f_{\kappa}$ approaches zero if $y_{0} \gg \delta_{\kappa}$. This function is even quite near zero one thermal penetration depth from the stack surface. Without applied flow, the total enthalpy flux equals the second-order enthalpy flux. Neglecting axial conduction in the fluid and the solid, Equation (22) simplifies to

$$
\dot{H}_{\text {tot }}=\dot{H}_{2}=-\frac{\delta_{x}}{4 y_{0}} p_{1}^{s} u_{1}^{s} A_{f u i d} T_{0} \beta[1-\Gamma]
$$

for $y_{0} \gg \delta_{k} \sim \delta_{v}$ and where the global normalized zeroth order temperature gradient along the direction of acoustic propagation is $\Gamma=\nabla T_{\text {ref }} / \nabla T_{\text {crit }}$ and $\nabla T_{c r i t}=\left(T \beta a \omega / c_{p}\right)_{r e f} \cot \left(X_{r e f} / \lambda\right)$. To this point, the discussion has followed the approach taken by Swift (1988). The results will now be modified for the presence of applied flow.

Equation (23) uses the convention: positive $\dot{H}_{2}$ flows along the stack in the direction of increasing $x$. This convention is used here and will be used in the remaining discussion along with the convention that heat into the stack and work out of the stack are positive real quantities. Since the second-order enthalpy flux for a refrigerator flows from cold to hot, $\dot{H}_{2}$ is negative. Without applied flow, the stack temperature is a nearly linear function of position $x$. The superposition of a steady flow through the stack, 
$d\left(\rho U_{a}\right) / d t=0$ and $u_{a} \ll<\left|u_{1}\right|$, adds another second order term to the total enthalpy flux equation

$$
\dot{H}_{t o t}=\dot{H}_{2}+\dot{H}_{0}
$$

where $\dot{H}_{0}=\rho_{0} c_{p} U_{a} T_{0}$. The applied flow moves toward the pressure node, so $\dot{H}_{0}$ is positive. If the stack is laterally well insulated the total enthalpy flux through it is independent of position

$$
\frac{d}{d x}\left[\dot{H}_{2}+\dot{H}_{0}\right]=0
$$

Defining the overall temperature gradient across the stack, $\nabla T_{\text {ref }}$, and the reference temperature, $T_{r e f}$, as reference constants such that $T_{r e f} \gg \nabla T_{\text {ref }} L$, this equation can be rewritten

$$
\frac{d}{d x}\left[\left(\frac{\dot{H}_{2}^{r e f}}{\nabla T_{r e f}}\right) \frac{d T_{0}}{d x}+\left(\frac{\dot{H}_{0}^{r e f}}{T_{r e f}}\right) T_{0}\right]=0
$$

Combining the reference constants into the second term on the left side of the equation produces a constant $\Xi=\left(\dot{H}_{0}^{\text {ref }} / \dot{H}_{2}^{\text {ref }}\right)\left(\nabla T_{\text {ref }} / T_{\text {ref }}\right)$ that contains the ratio of the applied-flow 
to the second-order enthalpy fluxes. The configuration considered in this example has $\dot{H}_{0}^{\text {ref }}>0, \dot{H}_{2}^{\text {ref }}<0$, and $\nabla T_{\text {ref }}<0$, so the sign of $\Xi$ is positive, giving the axial temperature profile the exponential bow shown by the solid curve in Figure 6. Expanding Equation (26) by substitution of Equation (23) and the definition of $\dot{H}_{0}$ at the reference conditions gives

$$
\frac{d^{2} T_{0}}{d x^{2}}+4 \frac{y_{0} \rho_{0} c_{p} U_{a}}{A_{\text {fuid }} \delta_{\kappa} p_{1}^{s} u_{1}^{s}} \frac{\nabla T_{\text {ref }} \nabla T_{\text {crit }}}{\left(\nabla T_{\text {ref }}-\nabla T_{\text {crit }}\right)} \frac{d T_{0}}{d x}=0
$$

Defining the exponential term $\Xi=\left(4 y_{0} \rho_{0} c_{p} U_{a} / A_{f f u d} \delta_{x} p_{1}^{s} u_{1}^{s}\right)\left(\nabla T_{\text {ref }} \nabla T_{\text {crit }} /\left(\nabla T_{\text {ref }}-\nabla T_{\text {crit }}\right)\right)$, and solving Equation (27) with the boundary conditions at $x=0, T=T_{H}$ and at $x=L$, $T=T_{C}$ gives the stack temperature $T_{0}$ as a function of axial position

$$
\frac{T_{0}-T_{H}}{T_{C}-T_{H}}=\frac{1-e^{-\Xi x}}{1-e^{-\Xi I}}
$$

The variable $\Xi$ contains the ratio of the applied-flow to the second-order enthalpy fluxes. This ratio can also be used to describe the other devices previously mentioned. If the applied flow direction is reversed, then the situation depicted in Figure 7 results. This device is called a thermoacoustic heater since its net effect is to move flow and thermoacoustic enthalpies toward the hot reservoir, $H$. 


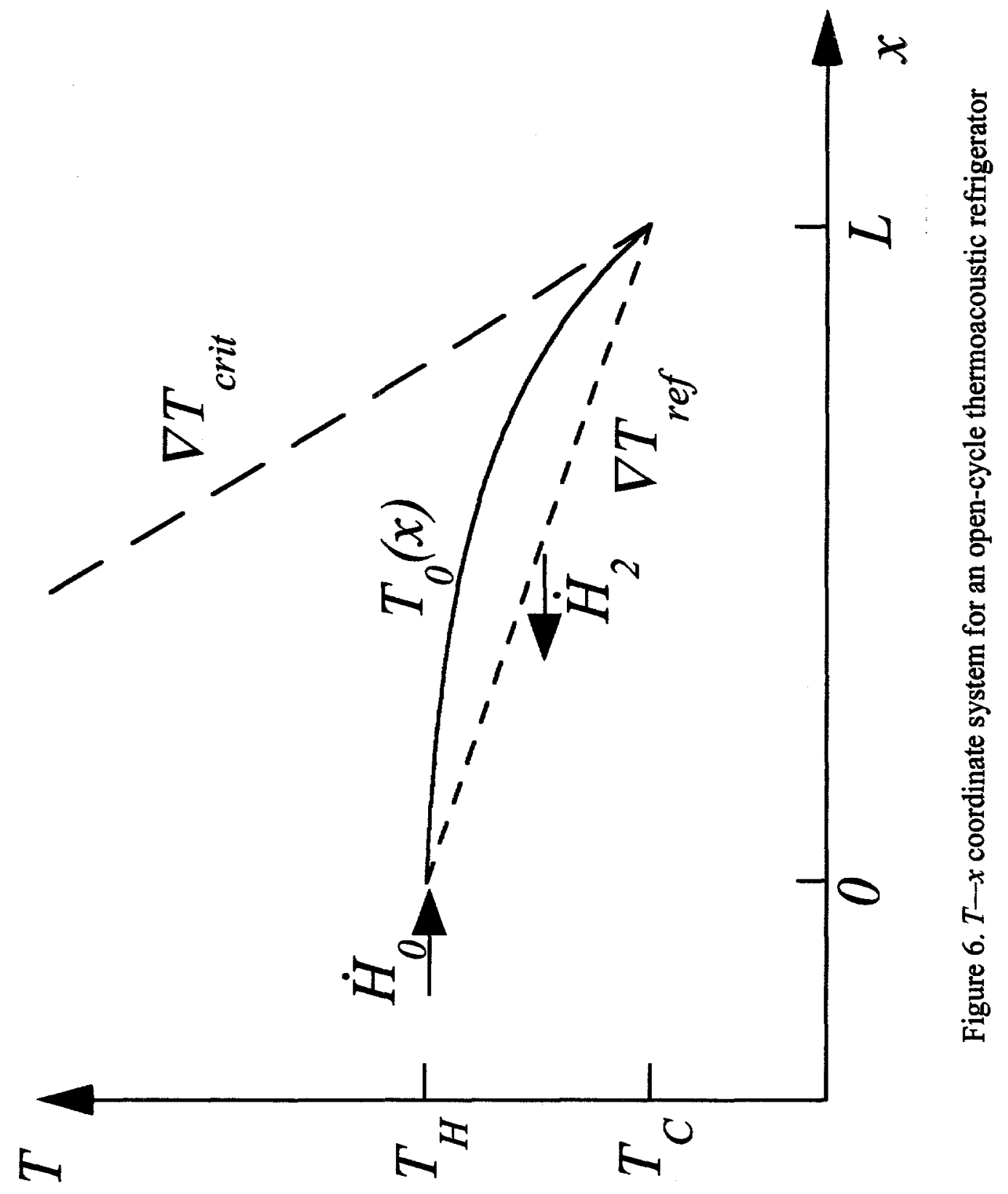




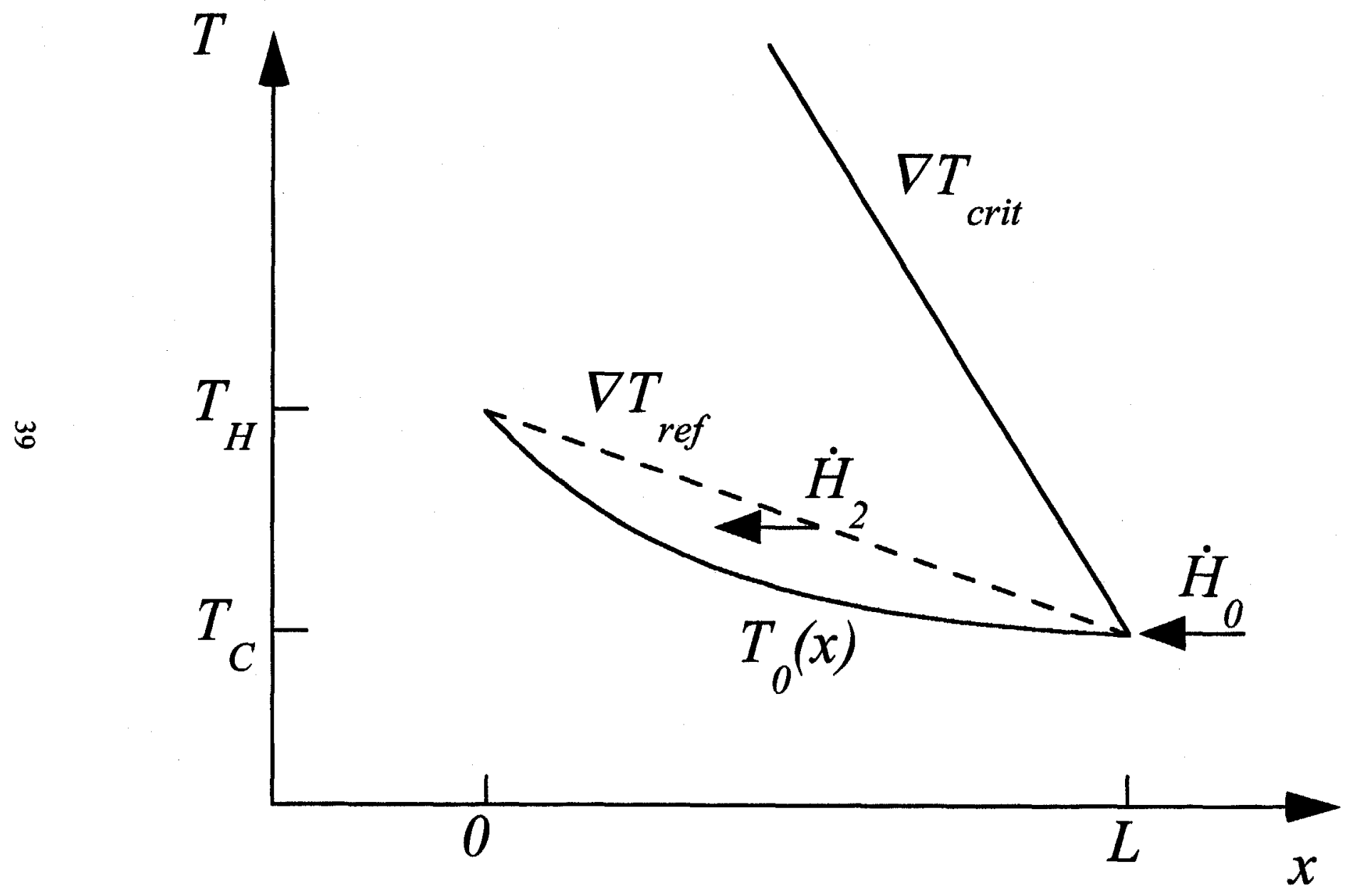

Figure 7. $T-x$ coordinate system for an open-cycle thermoacoustic heater 
Multiplying $\Xi$ by a suitable length scale (here taken to be the distance across the stack), using ideal gas assumptions, using Equation (23) for $\dot{H}_{2}$, using the dimensionless flow velocity $\left\langle\xi_{a}\right\rangle=U_{a} / f L A_{\text {fluid }}$, and arranging the result into a more natural order produces the dimensionless group

$$
\bar{\Xi}=4 \cdot\left(\frac{\gamma}{\gamma-1}\right)\left(\frac{p_{0}}{p_{1}^{s}}\right)\left(\frac{f L}{u_{1}^{s}}\right)\left\langle\xi_{a}\right\rangle\left(\frac{\nabla T_{r e f} \nabla T_{c r i t} L}{T_{r e f}\left(\nabla T_{r e f}-\nabla T_{c r i t}\right)}\right)\left(\frac{y_{0}}{\delta_{\kappa}}\right)
$$

By reversing the flow direction, Equation (28) can be used to describe the temperature distribution across an open-cycle thermoacoustic heater stack. In this dissertation, two types of thermoacoustic device will be tested: the refrigerator and the heater. For both these devices, $\dot{H}_{2}<0$. Two additional types of thermoacoustic device, the engine and the engine-heater, are also possible if the case $\dot{H}_{2}>0$ is considered. Values for short stack $\Xi$ corresponding to the experimental conditions are included in Appendix B. 


\section{Table 2. Inviscid Short Stack Assumptions}

1. Boundary layer flow over the stack plate.

2. Stack plate is much shorter than wavelength of the standing wave: $L<<\lambda$.

3. The magnitude of oscillatory velocity and pressure are uniform over the length of the stack plate: $\left|u_{1}\right|=$ constant and $\left|p_{1}\right|=$ constant .

4. Fluid has zero viscosity so oscillatory velocity does not depend on lateral distance from stack plate: $\mu=0$.

5. Stack plate temperature does not change appreciably with time: $d T_{0} / d t=0$.

6. Temperature dependence of thermophysical properties is neglected.

7. Stack plate has a given end-to-end temperature.

8. Neglect thermal conductivity along the length of the stack plate.

9. Neglect working fluid thermal conductivity in direction of sound propagation.

10. Mean fluid temperature is constant in the direction transverse to the stack plate and is the same as that of the stack plate: $d T_{0} / d y=0$.

11. Stack plate makes negligible thermal contact with fluid far from it.

12. Applied mass flow rate through stack is constant with time: $d\left(\rho U_{a}\right) / d t=0$.

13. Absolute fluid temperature is much greater than change in temperature across stack: $T_{0} \gg\left(d T_{0} / d x\right) L$.

14. Absolute temperature in stack is much greater than the magnitude of the firstorder temperature oscillations.

15. Working fluid behaves as an ideal gas: $p=\rho R T$.

16. Standing-wave phasing between $p_{1}$ and $u_{1}$.

17. Stack has negligible effect on the standing wave so the standing wave is described by simple trig functions which are positive real functions of $X$ : $p_{1}^{s}=\left|p_{A}\right| \cos \left(X_{\text {ref }} / \lambda\right), u_{1}^{s}=-i\left|p_{A}\right| /\left(\rho_{0} a\right) \sin \left(X_{\text {ref }} / \lambda\right)$.

18. Magnitude of the applied flow velocity is much less than the magnitude of the first order velocity.

19. The stack plate has infinite heat capacity.

20. The magnitude of applied velocity approaches zero. 


\section{Dimensionless Enthalpy-Flux Ratios}

The group $\bar{\Xi}$ derived from the inviscid short-stack assumptions gives a conceptually satisfying description of the relation between the stack enthalpy flux and the curvature in the stack temperature distribution. Unfortunately, this formulation is based on a number of fictitious notions. Among these is the assumption that the dimensionless enthalpy-flux ratio is constant along the entire stack. In practice, both the zero ${ }^{\text {th }}$-order and the secondorder enthalpy fluxes change considerably with position. This lack of realism severely limits the applicability of the short-stack definition of $\bar{\Xi}$. Two additional definitions for $\bar{\Xi}$ are suggested below that depend on knowledge of the stack temperature distribution, of the applied mass flow rate, and of the second-order enthalpy flux at one stack point. These latter approaches to $\bar{\Xi}$ are descriptive: the curvature in the temperature distribution is assumed known. The approach to the short-stack $\bar{\Xi}$ is (however imperfectly) predictive: the flow-induced temperature curvature is derived directly from other system properties. The relation of these latter definitions to mean temperature profile and thermodynamic performance is beyond the scope of this work.

A global definition of $\bar{\Xi}$ can be taken as the ratio of the total enthalpy extracted from the applied flow to the acoustically produced second-order enthalpy flux at one point in the stack. The enthalpy change in the applied flow as it moves through the stack is

$$
\Delta \dot{H}_{0}=\dot{H}_{0}^{H}-\dot{H}_{0}^{C}=\rho_{0} U_{a} c_{p}\left(T_{H}-T_{C}\right)
$$


Consistent with a global definition of $\bar{\Xi}$, a second-order enthalpy flux $\dot{H}_{2}$ at a single stack position can be used. For convenience, a point is selected at the heat-rejection end of the stack. For refrigerators or heat pumps the heat-rejection end is at " $H$ "; for engines and engine-heaters the heat-rejection end is " $C$ ". The global definition for $\bar{\Xi}$ is then

$$
\bar{\Xi}=\frac{\dot{H}_{0}^{H}-\dot{H}_{0}^{C}}{\dot{H}_{2}^{\text {ref }}}
$$

where the superscipt "ref" can be defined at either $H$ or $C$. This global definition requires knowledge of the temperature at the stack ends and the second order enthalpy flux at some position. For a refrigerator filled with an ideal gas this definition becomes

$$
\Xi=\frac{p_{0}}{T_{0}} \frac{U_{a}}{\dot{H}_{2}^{H}}\left(\frac{\gamma}{\gamma-1}\right)\left(T_{H}-T_{C}\right)
$$

The values of this ratio for the data presented in Chapter 4 are included in Appendix B. This global definition of $\bar{\Xi}$ is close to the short stack definition of $\bar{\Xi}$ in character, but with knowledge of $\dot{H}_{2}$ at some point in the stack, it is not limited by the short stack assumptions. However, this global $\bar{\Xi}$ still lacks information about the spatial distribution of enthalpy flux along the stack length. 
With applied flow the curvature of the mean temperature profile can change considerably with position. Near the hot end of a refrigerator stack the slope of the mean temperature profile can approach zero or even change sign. The magnitude of this slope increases as the applied flow moves toward the cold end of the stack. The local enthalpy change of the applied flow component is

$$
\Delta \dot{H}_{0}(x)=\frac{p_{0}}{T_{0}} U_{a}\left(\frac{\gamma}{\gamma-1}\right) \nabla T_{0}(x) L
$$

where the scale $L$ expresses the temperature change as if the local temperature gradient were continued along the stack length. For a stack with an adiabatic boundary and $\dot{H}_{2}$ and $\dot{H}_{0}$ known at one point, knowledge of the temperature distribution implies that $\dot{H}_{2}$ is known at all other points

$$
\dot{H}_{2}(x)=\dot{H}_{2}^{H}+\frac{p_{0}}{T_{0}} U_{a}\left(\frac{\gamma}{\gamma-1}\right)\left[T_{H}-T(x)\right]
$$

which suggests a definition for a local enthalpy ratio:

$$
\bar{\Xi}(x)=\frac{\left(p_{0} / T_{0}\right) U_{a}(\gamma / \gamma-1) \nabla T(x) L}{\dot{H}_{2}^{H}+\left(p_{0} / T_{0}\right) U_{a}(\gamma / \gamma-1)\left[T_{H}-T(x)\right]}
$$


The value of $\dot{H}_{2}$ can be computed accurately by solving the system of equations given in Appendix A. An inviscid approximation to $\dot{H}_{2}$ can also be found using Equation (23) (short stack approximation). Another approach to calculating $\dot{H}_{2}$ for non-zero viscosity is the use of the approximation of Equation (81) found in Swift (1988). Representative values for this ratio using the Chapter 4 data set and $\dot{H}_{2}$ computed by the method outlined in Appendix A are presented in Appendix B.

\section{Cycle Representation in Phase Space}

A $T-s-x$ pathline is described for an inviscid gas particle moving near an opencycle-thermoacoustic-refrigerator stack plate during its journey through a refrigerator stack. This pathline completely defines the thermodynamic state of the particle subject to the assumptions listed in Table 2. While the boundary-layer approximation is preserved in the definition of $\Xi$, it is relaxed somewhat when calculating the phase relation between Lagrangian temperature and entropy. A quarter-wavelength acoustic standing wave is oriented so that the applied flow moves with increasing $x$ toward the pressure node. The computation begins at $t=0$ when the standing wave is in its most compressed phase. A gas particle is selected over the hot heat exchanger at $x_{H}$, one displacement amplitude away from the stack hot end. The gas particle remains at a fixed distance $y_{0}$ above the surface of the stack plate and is followed as it moves through the stack from the hot to the cold heat exchanger. Computation ends after the mean position of the gas particle has 
passed into the cold heat exchanger and the standing wave is in its most expanded phase. This computation interval also defines the particle residence time in the stack. Five variables are used to describe the gas particle's movement and thermodynamic state

$$
\begin{aligned}
& \zeta=\zeta_{0}+\operatorname{Re}\left[\zeta_{1} e^{i \omega t}\right] \\
& u=u_{a}+\operatorname{Re}\left[u_{1} e^{i \omega x}\right] \\
& p=p_{0}+\operatorname{Re}\left[p_{1} e^{i \omega t}\right] \\
& T=T_{0}+\operatorname{Re}\left[T_{1} e^{i \omega t}\right] \\
& s=s_{0}+\operatorname{Re}\left[s_{1} e^{i \omega t}\right]
\end{aligned}
$$

where $\zeta$ represents the Lagrangian position of the fluid particle. The quantities $[\zeta, u, p, T, s]$ and $\left[\zeta_{0}, u_{a}, p_{0}, T_{0}, s_{0}\right]$ are real numbers while $\left[\zeta_{1}, u_{1}, p_{1}, T_{1}, s_{1}\right]$ are complex. Simple positive real trig functions can be used to spatially describe the standing wave between $0<X<\lambda / 4$. To simplify the math, all points along the stack are assumed to share the oscillatory pressure and velocity at a single position, $X_{r e f}$, in the standing wave. This assumption is reasonable since $\lambda / 4$ is typically much longer than the stack. The oscillatory pressure wave is $p_{1}=p_{1}^{s} e^{i(0)}$ where the positive-real variable $p_{1}^{s} \equiv p_{A} \cos \left(X_{\text {ref }} / \lambda\right)$ across the stack. Using the one-dimensional momentum equation, $i \omega \rho_{0} u_{1}=-d p_{1} / d X$, the $x$-component of oscillatory velocity can be found: $u_{1}=\left(p_{A} / \rho_{0} a\right) \sin \left(X_{r e f} / \lambda\right) e^{i(-\pi / 2)}$, where $u_{1}^{s} \equiv\left(p_{A} / \rho_{0} a\right) \sin \left(X_{r e f} / \lambda\right)$ at all points along the 
stack. These conventions allow Equations (36) through (40) to be evaluated for the path followed by the gas particle which first passes through $x_{H}$ and $y_{0}$.

$$
\begin{gathered}
\zeta\left(x_{H}, y_{0}, t\right)=u_{a} t+\frac{u_{1}^{s}}{\omega} \operatorname{Re}\left[e^{i(\omega t-\pi)}\right] \\
u\left(x_{H}, y_{0}, t\right)=u_{a}+u_{1}^{s} \operatorname{Re}\left[e^{i(\omega t-\pi / 2)}\right] \\
p\left(x_{H}, y_{0}, t\right)=p_{0}+p_{1}^{s} \operatorname{Re}\left[e^{i \omega t}\right]
\end{gathered}
$$

The phase shift in the complex exponential terms reflects the initial particle displacement toward the pressure antinode when the standing wave is in its most compressed phase. The material derivative for temperature can be expanded to calculate the temperature of the gas particle moving through the stack

$$
\frac{D T}{D t}=\frac{\partial T}{\partial t}+u \frac{\partial T}{\partial x}
$$

Substituting Equations (37) and (39) gives

$$
\frac{D T}{D t}=\frac{\partial T_{0}}{\partial t}+\frac{\partial \operatorname{Re}\left[T_{1} e^{i \omega t}\right]}{\partial t}+u_{a} \frac{\partial T_{0}}{\partial x}+u_{a} \frac{\partial \operatorname{Re}\left[T_{1} e^{i \omega t}\right]}{\partial x}+\operatorname{Re}\left[u_{1} e^{i \omega t}\right] \frac{\partial T_{0}}{\partial x}+\operatorname{Re}\left[u_{1} e^{i \omega t}\right] \frac{\partial \operatorname{Re}\left[T_{1} e^{i \omega t}\right]}{\partial x}
$$


The first term on the right side of the equation is eliminated in steady state. The last term is second order and can be eliminated as well. Since the magnitude of $u_{a}$ is effectively second order, the third and fourth terms on the right side of the equation are negligible. The first-order terms that remain are

$$
\frac{D T}{D t}=\frac{\partial \operatorname{Re}\left[T_{1} e^{i \omega x}\right]}{\partial t}+u_{a} \frac{\partial T_{0}}{\partial x}+\operatorname{Re}\left[u_{1} e^{i \omega t}\right] \frac{\partial T_{0}}{\partial x}+\operatorname{Re}\left[u_{1} e^{i \omega t}\right] \frac{\partial \operatorname{Re}\left[T_{1} e^{i \omega t}\right]}{\partial x}
$$

Integrating the material derivative with respect to time yields the temperature history of the gas particle at $x_{H}$ when $t=0$ :

$$
\begin{gathered}
T\left(x_{H}, y_{0}, t\right)=T_{0}\left(x_{H}, y_{0}\right)+\operatorname{Re}\left[T_{1} e^{i \omega x}\right]+u_{a} \frac{\partial T_{0}}{\partial x} t+\operatorname{Re}\left[\frac{u_{1}}{i \omega} e^{i \omega x}\right] \frac{\partial T_{0}}{\partial x}+ \\
\frac{R T_{0}}{a} \Psi_{1} \frac{\partial Z}{\partial x}\left[\frac{\Psi_{2}}{2 \omega} \sin ^{2} \omega t-\Psi_{3}\left(\frac{t}{2}-\frac{1}{4 \omega} \sin 2 \omega t\right)\right]
\end{gathered}
$$

where

$$
\begin{gathered}
Z=\frac{p_{1}^{s}}{p_{0}} \frac{R}{c_{p}} T_{0}+\frac{u_{1}^{s}}{\omega} \frac{\partial T_{0}}{\partial x}, \text { and } \\
\Psi_{1}=\frac{p_{A}}{p_{0}} \sin (x / \lambda) ; \Psi_{2}=1-e^{-y / \delta_{\kappa}} \cos \left(-y / \delta_{x}\right) ; \Psi_{3}=e^{-y / \delta_{\kappa}} \sin \left(-y / \delta_{\kappa}\right)
\end{gathered}
$$

The mean particle temperature and its spatial derivative are found from Equation (28): 


$$
T_{0}(x)=T_{H}+\left(T_{C}-T_{H}\right) \frac{1-e^{-\Xi x}}{1-e^{-\Xi L}}
$$

and

$$
\frac{\partial T_{0}}{\partial x}=\left(T_{C}-T_{H}\right) \frac{\Xi e^{-\Xi x}}{1-e^{-\Xi L}}
$$

The standing-wave velocity defined in Equation (42) is also used to modify the solution to a differential equation found in Swift (1988) that solves $T_{1}$

$$
T_{1}=\left[\frac{p_{1}^{s} R}{p_{0} c_{p}} T_{0}+\frac{u_{1}^{s}}{\omega} \frac{\partial T_{0}}{\partial x}\right]\left[1-e^{-(1+i) y / \delta_{x}}\right]
$$

Combining Equations (47)-(50) and using Equation (42) gives the Lagrangian temperature of the fluid particle as a function of time where the position term appearing in the exponential is $\zeta=u_{a} t$.

In a like fashion, eliminating terms higher than first order from the material derivative for entropy gives

$$
\frac{D s}{D t}=\frac{\partial \operatorname{Re}\left[s_{1} e^{i \omega t}\right]}{\partial t}+u_{a} \frac{\partial s_{0}}{\partial x}+\operatorname{Re}\left[u_{1} e^{i \omega t}\right] \frac{\partial s_{0}}{\partial x}+\operatorname{Re}\left[u_{1} e^{i \omega x}\right] \frac{\partial \operatorname{Re}\left[s_{1} e^{i \omega x}\right]}{\partial x}
$$


Integrating the entropy material derivative with respect to time and using the ideal gas relation for isobaric entropy change $s_{0}=s_{0}^{H}+c_{p} \ln \left(T_{0} / T_{0}^{H}\right)$ and its first order perturbation $s_{1}=\left(c_{p} / T_{0}\right) T_{1}-\left(\beta / \rho_{0}\right) p_{1}$ leaves

$$
\begin{aligned}
s\left(x_{t}, y_{0}, t\right)= & s\left(x_{H}, y_{0}\right)+\frac{c_{p}}{T_{0}} Z \Psi_{2} \cos (\omega t)+\frac{c_{p}}{T_{0}} Z \Psi_{3} \sin (\omega t)- \\
& R \Psi_{1} \cos (\omega t)+\frac{u_{a} c_{p}}{T_{0}} \frac{\partial T_{0}}{\partial x} t-\frac{R T_{0}}{a \omega} \Psi_{1} \frac{c_{p}}{T_{0}} \frac{\partial T_{0}}{\partial x} \cos (\omega t)+ \\
& \frac{R T_{0}}{a} \Psi_{1}\left[\left(c_{p} \Psi_{2} \Lambda_{1}+\frac{R \Psi_{1}}{\lambda}\right)\left(\frac{1}{2 \omega} \sin ^{2}(\omega t)\right)+\left(c_{p} \Psi_{3} \Lambda_{1}\right)\left(\frac{t}{2}-\frac{1}{4 \omega} \sin (2 \omega t)\right)\right]
\end{aligned}
$$

where $\Lambda_{1}=-R \frac{p_{A}}{p_{0}}\left[\frac{1}{c_{p} \lambda} \sin (x / \lambda)+\frac{1}{\omega a \lambda} \cos (x / \lambda) \frac{\partial T_{0}}{\partial x}+\frac{1}{\omega a} \sin (x / \lambda) \frac{\partial^{2} T_{0}}{\partial x^{2}}\right]$

Representative conditions in the refrigerator stack described in Chapter 4 are given in Table 3. The reference mean entropy $s_{0}^{H}$ is evaluated at $273 \mathrm{~K}$. Figures 8 , 9 , and 10 show $T-s, T-x$, and $s-x$ views of the above equations using Table 3 values. These plots, dubbed the "flow slinky," trace the movement of a particle one thermal penetration depth above the surface of the stack plate in its journey across a refrigerator stack. This distance above the stack plate was selected since most of the work and heat transport occurs near there. 
Table 3. Representative Refrigerator Stack Conditions

\begin{tabular}{lll}
\hline \multicolumn{1}{c}{ Description } & Symbol & \multicolumn{1}{c}{ Value } \\
\hline Stack Length & $L$ & $0.1524 \mathrm{~m}$ \\
Working Fluid & - & $92 \% \mathrm{He}-8 \%$ Ar by volume \\
Specific Heat Ratio & $\gamma$ & $5 / 3$ \\
Operating Frequency & $f$ & $93.94 \mathrm{~Hz}$ \\
Mean Pressure & $p_{0}$ & $322,280 \mathrm{~Pa}$ \\
Stack Oscillatory Pressure & $p_{1}^{s}$ & $5816 \mathrm{~Pa}$ \\
Hot-end Temperature & $T_{H}$ & $307.89 \mathrm{~K}$ \\
Cold-end Temperature & $T_{C}$ & $300.56 \mathrm{~K}$ \\
Stack Oscillatory Velocity & $u_{1}^{s}$ & $5.69 \mathrm{~m} / \mathrm{s}$ \\
Applied Flow Velocity & $u_{a}$ & $0.37 \mathrm{~m} / \mathrm{s}$ \\
\hline \hline
\end{tabular}

In three-space the $T-s-x$ path forms a series of elliptically shaped helical spirals. For the $T-s$ representation shown in Figure 8 the phase of temperature leads entropy and the spirals move from right to left in a counter-clockwise motion, indicating that the fluid in the vicinity of the particle absorbs work from its surroundings. The entropy of the fluid particle decreases in its traverse across the stack as does its temperature. The magnitude of the temperature oscillation remains nearly constant while the magnitude of the entropy oscillation doubles as the particle moves from hot to cold. The particle begins its path at the phase of maximum compression. The gas parcel does work on its surroundings as it expands while being displaced toward the pressure node. As the 


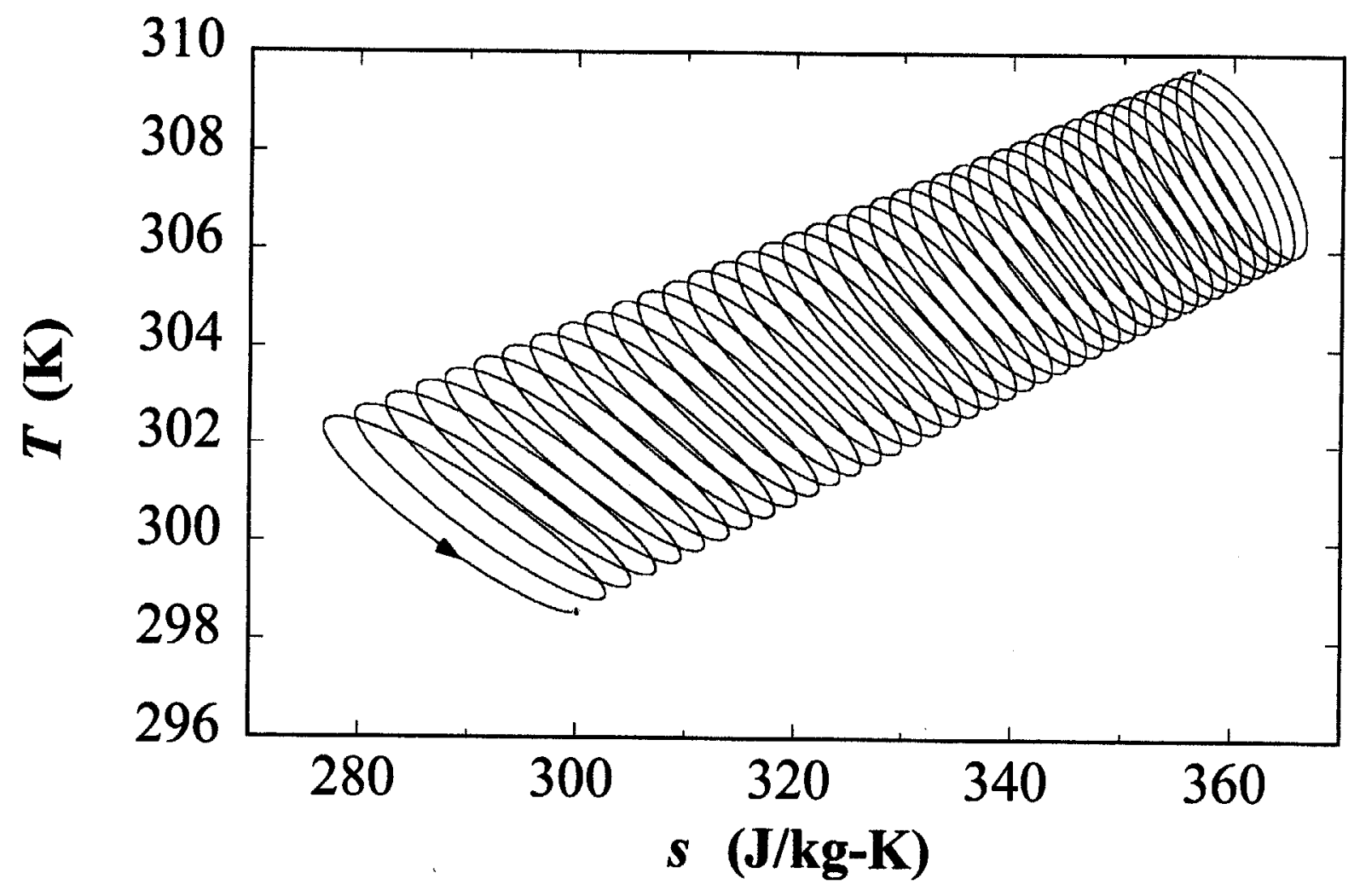

Figure 8. $T-s$ representation of an inviscid open thermoacoustic refrigeration cycle 
expansion reduces the parcel temperature below that of the stack plate, the parcel begins to absorb heat from the plate. The parcel absorbs external externally generated work and is compressed and displaced toward the velocity node. Heat is then transferred from the compressed parcel to the stack plate. The next cycle path passes over the preceding one, making incremental progress toward the cold end of the stack. As the parcel moves from the hot to the cold ends of the stack there is a marked $20^{\circ}$ counterclockwise change in the angle of the cycle ellipsoid in $T-s$ space.

The orientation of the cycle ellipsoid in $T-s$ space gives an indication of the distance of the particle normal to the stack plate. The path lines for particles in good thermal contact with the stack plate approach the horizontal (isothermal orientation). For gas particles near the stack plate the temperature and entropy oscillations are in phase. About one thermal penetration depth from the stack plate, the gas around the particle expands at high temperature and contracts at low temperature, producing refrigeration. For particles far away from the stack plate, the spirals move closer to the vertical (toward the isentropic orientation). However, this movement toward the isentropic orientation is limited by the movement of the particle down the mean temperature gradient. For gas particles far away from the stack plate, the temperature and entropy oscillations are again in phase (i.e. there is no movement of heat toward the hot end of the stack).

The path line on the $T-x$ plot shown in Figure 9 begins in the particle's phase of greatest compression when the particle is nearest the velocity node and at its highest temperature. There is a slight outward bow in the curvature of the mean temperature 


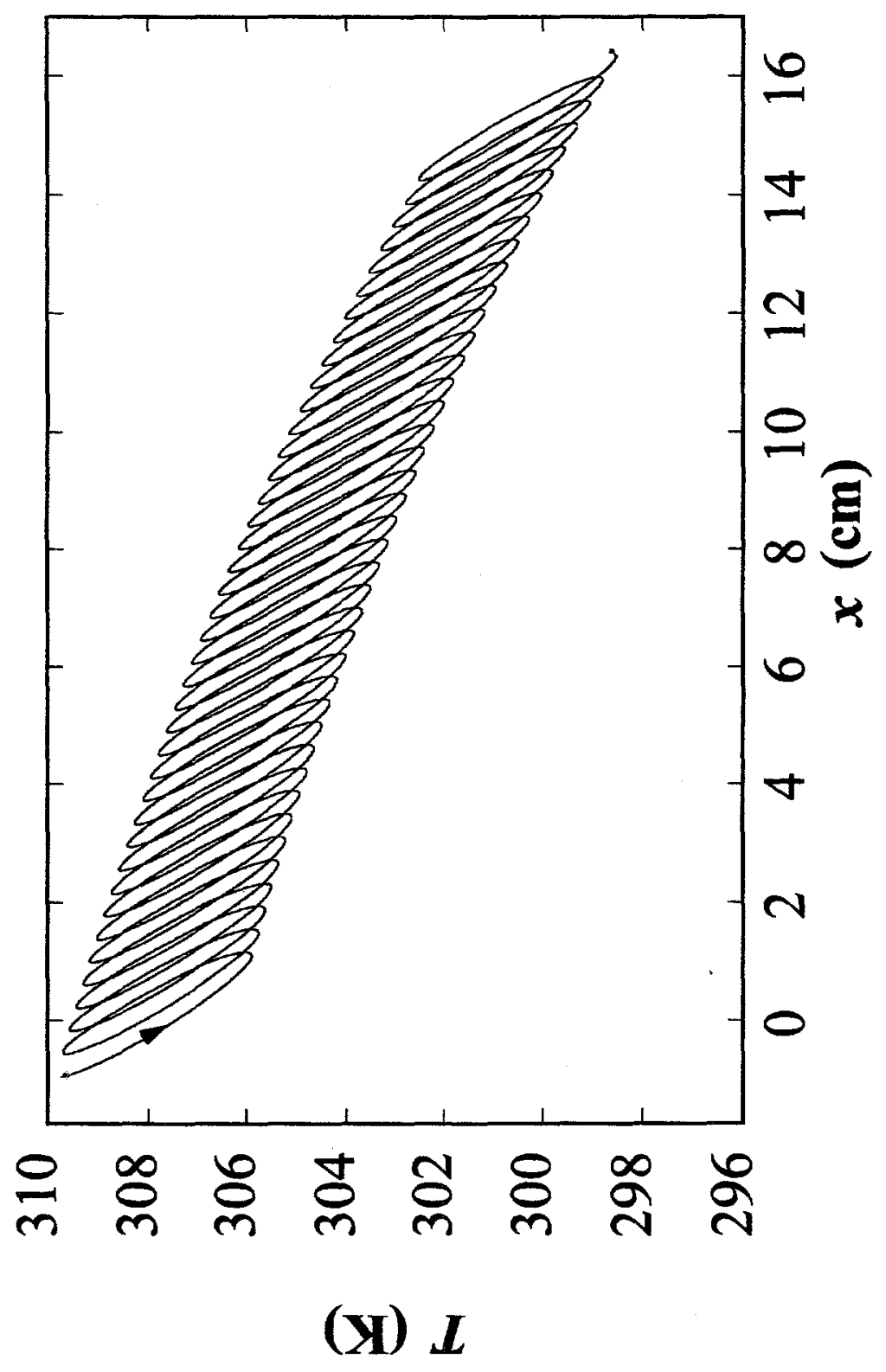

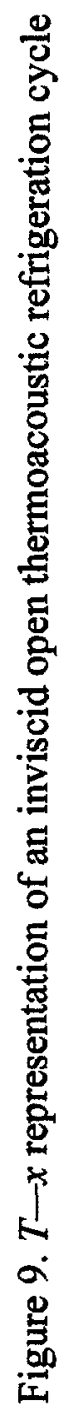

(y) $\boldsymbol{L}$ 
profile formed from the balance of the zeroth-order and second-order enthalpy fluxes contained in inviscid $\Xi$. Again, the particle motion is counter-clockwise. The path line is tracked until the particle reaches a point of maximum expansion at the cold end of the stack. As in the $T-s$ plot, the temperature oscillation magnitude remains constant throughout the stack, as do the magnitude of the displacement oscillations. There is a $5^{\circ}$ counterclockwise change in the angle of the cycle ellipsoid from the beginning to end of the stack.

An $s-x$ projection of the preceding plots is shown in Figure 10, which completes the orthogonal set. Common with the $T-x$ plot is an exponential curvature in the mean entropy profile with position. The tendency for cycle loops to overlap is most pronounced toward the stack entrance. The cycle moves in a counter-clockwise fashion with decreasing mean entropy as the end of the stack is reached. As with the $T-s$ plot, there is a $20^{\circ}$ counterclockwise change in the cycle ellipsoid angle as the parcel moves from stack entrance to stack exit. The entropy gradient is also larger toward the cold end of the stack. 


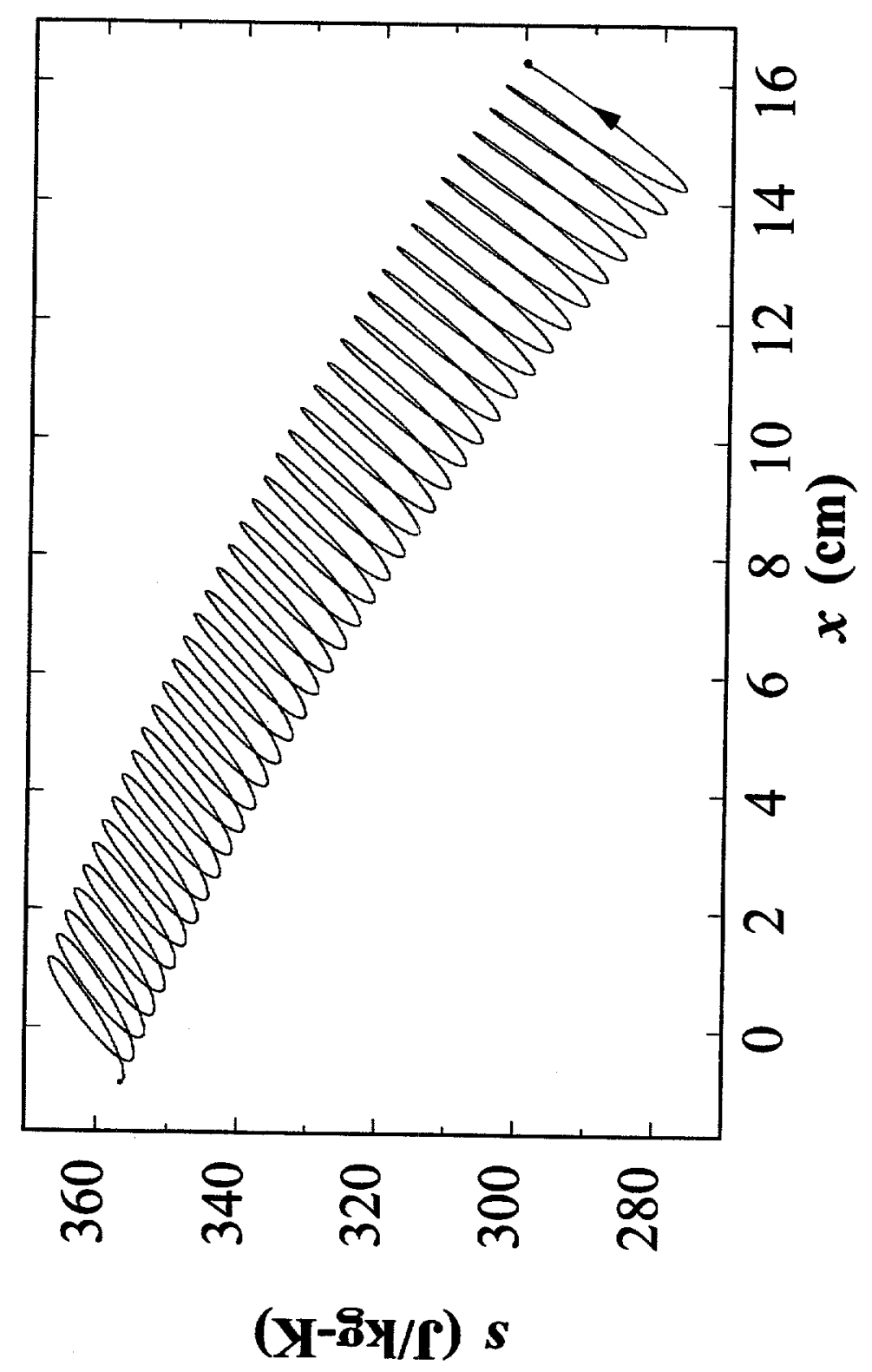

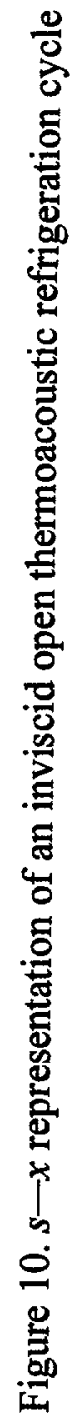




\section{Some Performance Indices}

Consider an open thermodynamic system containing a simple compressible substance, which is in contact with thermal energy reservoirs that are not necessarily at the same temperature as the applied flows entering or leaving the system. A general expression for the principle of energy conservation for this system is given by

$$
\dot{Q}-\dot{W}+\int_{A_{i}}\left(h+e_{k}+e_{p}\right) \rho(\mathbf{v} \cdot \mathbf{n}) d A-\int_{A_{e}}\left(h+e_{k}+e_{p}\right) \rho(\mathbf{v} \cdot \mathbf{n}) d A=\frac{d}{d t} \int_{V} e \rho d V
$$

where heat into the system and work out of the system are treated as positive quantities. If the system operates in steady state, the time rate of change of any property of the system is zero. Since the total energy of the mass within the system is an property of the system, there can be no change in the total energy in the system with time. For the case of uniform applied flow across the system's inlet and exit cross sectional areas, the energy conservation equation reduces to

$$
\dot{Q}-\dot{W}+\sum_{\text {inlet }} \dot{m}\left(h+e_{k}+e_{p}\right)-\sum_{\text {exit }} \dot{m}\left(h+e_{k}+e_{p}\right)=0
$$

The kinetic and potential energy for low-speed gas flows are small compared with the enthalpy. For a system with a single inlet and exit, the energy conservation equation becomes 


$$
\dot{Q}-\dot{W}+\dot{m}\left(h_{i}-h_{e}\right)=0
$$

The performance of a thermodynamic cycle can be generally described by the ratio of a desired kind of energy transfer achieved to an energy transfer that exacts a limiting cost or penalty on the device or system. This performance definition includes, but is somewhat broader than, what is commonly called first-law efficiency. This definition will be referred to as the "performance index" for a thermodynamic device or system. An example of a performance index is the familiar coefficient of performance for a refrigerator combining heat absorbed $\dot{Q}_{c}$ from a low temperature reservoir at $T_{c}$ with the cooling of an applied flow. In this case the sought energy transfer is sum of the absorption of heat $\dot{Q}_{C}$ at $T_{C}$ and the absorption of enthalpy from the applied flow as it passes through the system from $T_{H}$ to $T_{C}$. The cycle penalty is the work needed to produce the refrigeration. For this system Equation (5) expands to

$$
\dot{Q}_{H}+\dot{Q}_{C}-\dot{W}+\dot{m}\left(h_{H}-h_{C}\right)=0
$$

Solving for work in Equation (6), the performance index for the refrigerator becomes 


$$
\beta_{1}=\frac{-\left[\dot{Q}_{C}+\dot{m}\left(h_{H}-h_{C}\right)\right]}{\dot{Q}_{H}+\dot{Q}_{C}-\dot{m}\left(h_{C}-h_{H}\right)}
$$

The Second Law of thermodynamics states that the entropy generated by a system and its surroundings must be positive or zero. A general equation for the total rate of change of entropy of a system and its surroundings is

$$
\left(\frac{d S}{d t}\right)_{\text {total }}=\int_{A_{e}} s \rho(\mathbf{v} \cdot \mathbf{n}) d A-\int_{A_{i}} s \rho(\mathbf{v} \cdot \mathbf{n}) d A+\frac{d}{d t} \int_{V} s \rho d V+\sum_{k} \frac{\dot{Q}_{k}}{T_{k}} \geq 0
$$

where the quantity $\dot{Q}_{k} / T_{k}$ is the heat transferred to the reservoirs. For a system in steady state with uniform applied flow across a single inlet and exit, Equation (58) reduces to

$$
\left(\frac{d S}{d t}\right)_{\text {toral }}=\dot{m}\left(s_{e}-s_{i}\right)+\sum_{k} \frac{\dot{Q}_{k}}{T_{k}} \geq 0
$$

Applying the heat flows between the system and its surroundings, the equation reduces to

$$
\dot{m}\left(s_{C}-s_{H}\right)-\frac{\dot{Q}_{H}}{T_{H}}-\frac{\dot{Q}_{C}}{T_{C}} \geq 0
$$


For a reversible system Equation (60) is an equality. Combining Equation (57) with Equation (60) for a reversible system, by eliminating the waste heat $\dot{Q}_{H}$, yields a performance index for a reversible refrigerator with uniform, steady applied flow

$$
\beta_{1, \text { Camot }}=\frac{-\left[\dot{Q}_{C}+\dot{m}\left(h_{H}-h_{C}\right)\right]}{\dot{Q}_{C}\left(1-T_{H} / T_{C}\right)-\dot{m}\left(h_{C}-h_{H}\right)+\dot{m} T_{H}\left(s_{C}-s_{H}\right)}
$$

The limiting case with no flow yields the familiar result for coefficient of performance for a closed-cycle refrigerator. Less familiar is the result for finite flow but no cold end heat absorption. Other performance indexes for the simple open-cycle thermoacoustic devices discussed in Chapter 2 are given in Table 4. The performance indices in Table 4 can be combined to yield the Second Law effectiveness for their respective devices. For a refrigerator, the Second Law effectiveness is the ratio of the reversible to actual work necessary to perform a given task. For an engine, it is the ratio of actual work to reversible work for a given amount of thermal energy supplied. The value of Second Law effectiveness is zero in the totally irreversible limit and unity in the reversible limit for both device types. 
Table 4. Some Indices of Performance
Device
First Law Performance Index
Carnot Perfomance Index
Second Law Effectiveness

\section{Inverse Prime Movers}

Refrigerator

Figure 5(A)

Mode \#1

Refrigerator

Figure 5(A)

Mode \#2

2

\section{Heater \\ Figure 5(B) \\ Mode \#3 \\ Heater (heat pump) \\ Figure 5(B) \\ Mode \#4 \\ Prime Movers}

Engine (conventional)

Figure 5(C)

Mode \#1

Engine

Figure 5(C)

Mode \#2

$$
\beta_{1}=\frac{-\left[\dot{Q}_{C}+\dot{m}\left(h_{H}-h_{c}\right)\right]}{\dot{Q}_{H}+\dot{Q}_{C}+\dot{m}\left(h_{H}-h_{c}\right)} \quad \beta_{1, \text { Carnot }}=\frac{-\left[\dot{Q}_{C}+\dot{m}\left(h_{H}-h_{C}\right)\right]}{\dot{Q}_{C}\left(1-T_{H} / T_{c}\right)-\dot{m}\left(h_{C}-h_{H}\right)+\dot{m} T_{H}\left(s_{C}-s_{H}\right)}
$$

$\varepsilon_{\mathrm{i}}=\frac{\dot{Q}_{C}\left(1-T_{H} / T_{C}\right)-\dot{m}\left(h_{C}-h_{H}\right)+\dot{m} T_{H}\left(s_{C}-s_{H}\right)}{\dot{Q}_{H}+\dot{Q}_{C}-\dot{m}\left(h_{C}-h_{H}\right)}$

$\beta_{2}=\frac{\dot{Q}_{H}}{\dot{Q}_{H}+\dot{Q}_{C}-\dot{m}\left(h_{C}-h_{H}\right)}$

$\beta_{2, \text { Carnot }}=\frac{\dot{Q}_{H}}{\dot{Q}_{H}\left(1-T_{c} / T_{H}\right)-\dot{m}\left(h_{C}-h_{H}\right)+\dot{m} T_{C}\left(s_{c}-s_{H}\right)}$

$\varepsilon_{2}=\frac{\dot{Q}_{H}\left(1-T_{c} / T_{H}\right)-\dot{m}\left(h_{C}-h_{H}\right)+\dot{m} T_{C}\left(s_{C}-s_{H}\right)}{\dot{Q}_{H}+\dot{Q}_{C}-\dot{m}\left(h_{C}-h_{H}\right)}$

$\bar{\varepsilon}_{1}=\frac{\dot{Q}_{C}\left(1-T_{H} / T_{C}\right)-\dot{m}\left(h_{C}-h_{H}\right)+\dot{m} T_{H}\left(s_{C}-s_{H}\right)}{\dot{Q}_{H}+\dot{Q}_{C}-\dot{m}\left(h_{C}-h_{H}\right)}$

$\bar{\beta}_{1}=\frac{-\dot{Q}_{C}}{\dot{Q}_{H}+\dot{Q}_{c}-\dot{m}\left(h_{C}-h_{H}\right)}$

$\bar{\beta}_{1, \text { Carnot }}=\frac{-\dot{Q}_{C}}{\dot{Q}_{C}\left(1-T_{H} / T_{C}\right)-\dot{m}\left(h_{C}-h_{H}\right)+\dot{m} T_{H}\left(s_{C}-s_{H}\right)}$

$\bar{\beta}_{2, \text { Carnot }}=\frac{\dot{Q}_{H}+\dot{m}\left(h_{c}-h_{H}\right)}{\dot{Q}_{H}\left(1-T_{c} / T_{H}\right)-\dot{m}\left(h_{c}-h_{H}\right)+\dot{m} T_{C}\left(s_{C}-s_{H}\right)}$

$\bar{\beta}_{2}=\frac{\dot{Q}_{H}+\dot{m}\left(h_{C}-h_{H}\right)}{\dot{Q}_{H}+\dot{Q}_{c}-\dot{m}\left(h_{C}-h_{H}\right)}$

$\bar{\varepsilon}_{2}=\frac{\dot{Q}_{H}\left(1-T_{c} / T_{H}\right)-\dot{m}\left(h_{C}-h_{H}\right)+\dot{m} T_{C}\left(s_{C}-s_{H}\right)}{\dot{Q}_{H}+\dot{Q}_{C}-\dot{m}\left(h_{C}-h_{H}\right)}$
$\eta_{1}=\frac{\dot{Q}_{H}+\dot{Q}_{C}-\dot{m}\left(h_{C}-h_{H}\right)}{\dot{Q}_{H}}$

$\eta_{1, \text { Camot }}=\dot{Q}_{H}\left(1-T_{C} / T_{H}\right)-\dot{m}\left(h_{C}-h_{H}\right)+\dot{m} T_{C}\left(s_{C}-s_{H}\right)$

$\varepsilon_{1}=\frac{\dot{Q}_{H}+\dot{Q}_{C}-\dot{m}\left(h_{C}-h_{H}\right)}{\dot{Q}_{H}\left(1-T_{C} / T_{H}\right)-\dot{m}\left(h_{C}-h_{H}\right)+\dot{m} T_{C}\left(s_{C}-s_{H}\right)}$

$\eta_{2}=\frac{\dot{Q}_{H}+\dot{Q}_{C}-\dot{m}\left(h_{C}-h_{H}\right)}{-\dot{Q}_{C}}$

$\eta_{2, \text { Carnot }}=\dot{Q}_{C}\left(T_{H} / T_{C}-1\right)+\dot{m}\left(h_{C}-h_{H}\right)-\dot{m} T_{H}\left(s_{C}-s_{H}\right)$
$\varepsilon_{2}=\frac{\dot{Q}_{H}+\dot{Q}_{C}-\dot{m}\left(h_{C}-h_{H}\right)}{\dot{Q}_{C}\left(1-T_{H} / T_{C}\right)-\dot{m}\left(h_{C}-h_{H}\right)+\dot{m} T_{H}\left(s_{C}-s_{H}\right)}$ 


\section{CHAPTER 3}

\section{APPLIED FLOW REFRIGERATOR AND EXPERIMENT}

A schematic of the thermoacoustic refrigerator built for these measurements is shown in Figure 11. The refrigerator consisted of a toroidal resonator with a total volume of $0.0915 \mathrm{~m}^{3}$ filled with $92 \%$ helium, $8 \%$ argon mixture at 3.23-bars mean pressure. The refrigerator was also successfully operated with 0.8 bar air, but the helium-argon mixture provided larger, more accurately measurable cooling power. Four loudspeakers drove a 94-Hz fundamental resonance, with a full wavelength around the torus, with the loudspeaker pair on one side of the resonator moving $\pi$ out of phase from the loudspeaker pair on the other side. The pressure nodes of the standing wave (marked " $N "$ ) divided the resonator into symmetric halves. The velocity nodes of the standing wave were located in the enclosure behind each loudspeaker. The resonator and stack assemblies absorbed acoustic power generated by the loudspeakers. The stacks were positioned in the standing wave at a point close enough to the velocity node to reduce viscous losses yet far enough away to allow formation of a temperature gradient in the 


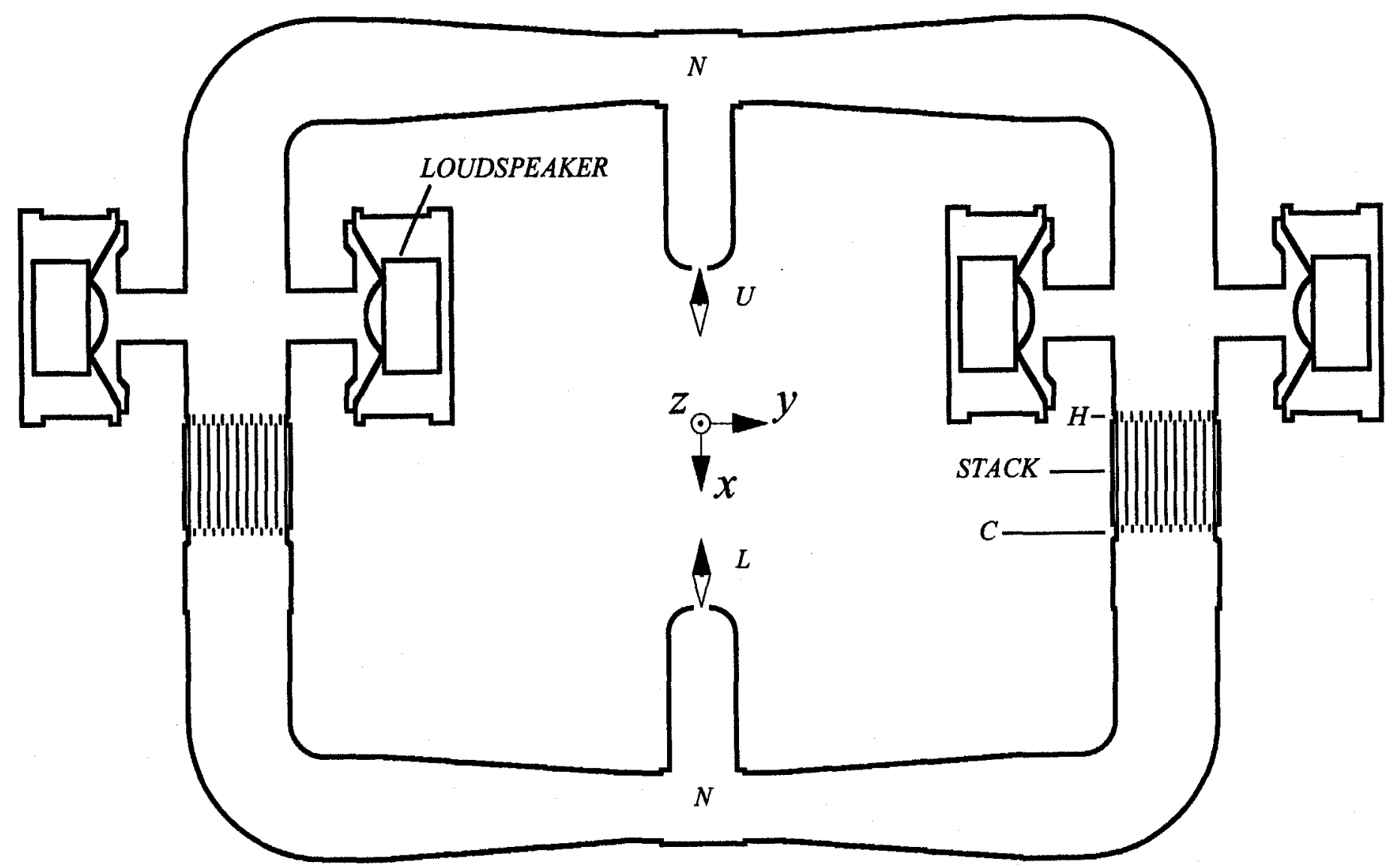

Figure 11. Schematic of a full wavelength thermoacoustic refrigerator with an externally applied flow. Labeled " $N$ " are the locations of the pressure nodes of the standing wave. " $H$ " indicates a room temperature heat exchanger and " $C$ " indicates a cold heat exchanger. The entrance and exit of the applied flow for the refrigerator is indicated by heavy arrows near the nodes. The cold heat exchangers are unnecessary for cooling the gas. 
standing wave. To ensure convective stability, the stacks were placed below the velocity nodes so that the heat-absorption end was at the lowest point. This toroidal configuration also allowed the refrigerator to operate quietly when its pressure nodes were open to the environment.

\section{Loudspeaker System}

The intent of this experiment was to demonstrate the operating principles of the open thermoacoustic cycle, not a practical device. Loudspeakers are typically designed to have an acoustic output response independent of frequency over their operating range. This tends to produce less-than-optimal electrical-to-acoustic conversion efficiency at a single operating frequency. Repeated efficiency measurements were taken on these loudspeakers during refrigerator tests. At no point did the electrical to acoustic conversion efficiency exceed $45 \%$. More-efficient linear motors, tuned to specific frequencies, have recently demonstrated electrical to acoustic conversion efficiencies of better than $90 \%$. Such motors might be useful in a practical mechanically driven opencycle thermoacoustic system. However, the loudspeakers used were ideally suited to a low-cost proof of principle experiment.

Four loudspeakers [1] supplied acoustic power to the resonator. Speaker pairs were contained within a hermetically sealed aluminum pod with penetrations for power leads and water lines. Each speaker was factory-equipped with a voice-coil-to-gas heat removal system. The forward motion of the speaker diaphragm created a rarefaction that allowed 
cooler ambient gas to enter the magnet gap and flow around the voice coil. The reverse motion ejected the heated gas behind the speaker magnet where it mixed with the surrounding gas and was cooled. Yet, with the restricted space behind each magnet and with the large currents applied to the voice coil, more cooling was needed to keep the voice coil at an acceptable temperature. Under the speaker cone, a plate was attached to the pole magnet to cover a pre-existing cavity. Two holes were then drilled into the magnet cavity from the rear. The holes allowed cooling water to pass through the cavity, putting flowing water in direct contact with the magnet, which regulated the magnet and voice coil temperatures. The temperatures of the entering and exiting flows were measured with Type $\mathrm{K}$ thermocouples (marked " $T \Gamma$ " and “TO" in Figure 17) [2]. A variable-area rotameter indicated the water flow rate through each speaker cavity [3]. Flow rate and temperature data recorded during refrigerator tests were later used in a system energy balance calculation.

A function generator [4] served as the sinusoidal signal source for the refrigerator. The signal source provided both frequency and amplitude control to the overall system. Four separate microphone mixers shared the function generator source signal. Each mixer controlled the electric power delivered to one loudspeaker. The signal leaving each mixer was fed into a power amplifier, with the amplified signal then routed to a loudspeaker through coaxial cable. To maintain the $\pi$ difference in phasing between sides, the signal polarity to the loudspeaker pair on one side of the resonator was reversed with respect to the opposing pair. 
The properties of each loudspeaker were characterized by measuring $B \ell$-product, mechanical resistance, electrical resistance, voice-coil inductance, and diaphragm stiffness. The $B \ell$-product of each speaker was measured by applying increasing amounts of weight to the loudspeaker diaphragm and increasing the direct current applied to the voice coil while keeping the diaphragm stationary. Diaphragm position was monitored with an optical sensor [5]. The slope of the weight-current curve yielded $B \ell$-product. The voice coil was placed in series with a signal source and a known capacitance. The inductance of a voice coil was found by measuring the electrical resonance frequency in a circuit with known capacitance. To prevent $B \ell v$ voltage contribution during the inductance measurement the voice coil was attached to the magnet with epoxy to keep it stationary. Speaker stiffness-to-mass ratio and mechanical resistance were found by measuring the electromechanical resonance frequency while operating the speakers in vacuum. Manufacturer-supplied data [6] was assumed for the mass of the moving diaphragm. Some variability was found in these measured properties from speaker to speaker, but none of these properties greatly differed from the manufacturer's specifications. Strong temperature dependence was noted in diaphragm stiffness. Stiffness measurements were taken at several temperatures to account for this dependence. These data were later used in the acoustic approximation calculation.

The rms electrical power supplied to each loudspeaker was measured with a dynamic-signal analyzer [7]. These measurements were used to calculate loudspeaker electric to acoustic efficiency and for the overall-system power balance. Two signals 
were used to measure the power supplied to each loudspeaker. The first signal was rms voltage measured across the loudspeaker terminals. The second signal was rms voltage measured across a precision resistor that sensed current supplied to the loudspeaker. During loudspeaker operation, this resistor was kept at $15^{\circ} \mathrm{C}$ by water cooling. Resistance measurements of the four precision resistors were made at $15^{\circ} \mathrm{C}$ with a potentiometer [8]. The measured values of these resistances were typically $0.1000 \Omega$. These signals were combined in the signal analyzer which computed the power-proportional cross spectrum of the terminal and shunt-rms-voltage signals. These measurements were made with a dynamic signal analyzer in swept-sine mode scanning a narrow range around the operating frequency. The electrical power supplied to each speaker was then found using the relation

$$
\dot{W}_{\text {elec }}=\frac{1}{2} \operatorname{Re}[I \tilde{E}]=\frac{\left|E_{\text {temminal }}\right| E_{\text {shumt }} \mid}{2 R_{\text {shurt }}} \cos \left(\theta_{\text {shumt }}-\theta_{\text {temminal }}\right)
$$

For the test results presented in Chapter 4 the phase difference between terminal and shunt voltage was typically $5.7^{\circ}$. With manufacturer-supplied uncertainty intervals [9] for phase and voltage measurements, and the uncertainty in the measurement of shunt voltage, the overall uncertainty interval in the electrical power measurement was estimated to be $\pm 4.6 \%$ of reading (Kline and McClintock, 1953). 
Due to these loudspeaker property variations, some difference was found between the acoustic power delivered to the resonator by each loudspeaker at a given terminal voltage. To reduce the difference, microphone mixers were used to control the terminal voltage at each loudspeaker so that each resonator side produced nearly equal amounts of acoustic power. Balancing was also found to increase the loudspeakers' overall electrical to acoustic efficiency. After mixer adjustment, the side-to-side difference in acoustic power delivered to the resonator was typically less than $2 \%$. The balance of the loudspeaker system was checked periodically. This balance was found to remain stable over a surprising range of resonator dynamic pressure amplitude.

Accelerometers attached to loudspeaker diaphragms have been previously used (Hofler, 1988) to measure the volume velocity used to calculate the experimental acoustic power delivered to a thermoacoustic resonator. A simpler method to measure volume velocity was used in this experiment, requiring only measurement of complex pressure on either side of a loudspeaker diaphragm. The average gas volume behind the speaker diaphragm was obtained by measurement. The mean pressure on either side of the loudspeaker diaphragm was assumed equal. The gas held in the volume behind the diaphragm was compressed and rarified by the diaphragm and can be assumed to follow an isentropic path. Using these assumptions, the diaphragm oscillatory volumetric velocity can be found by measuring the oscillatory pressure behind the loudspeaker diaphragm. The volumetric velocity of the speaker diaphragm is related to isentropic pressure-volume relation for the gas behind the loudspeaker diaphragm 


$$
p V^{\gamma}=\text { constant }
$$

Differentiating Equation (63) yields

$$
p_{0} d\left(V^{\gamma}\right)+V_{0}^{\gamma} d p=0
$$

Expanding Equation (64) and taking differential quantities to be first order perturbations yields

$$
p_{0} \gamma V_{0}^{\gamma-1} V_{1}+V_{0}^{\gamma} p_{1}=0
$$

The volumetric displacement amplitude, $V_{1}$, of the diaphragm is then only a function of the oscillatory pressure, $p_{1}$, in the volume behind the diaphragm:

$$
V_{1}=-V_{0} \frac{1}{\gamma} \frac{p_{1, B a c k}}{p_{0}}
$$

using $U_{1}=i \omega V_{1}$ and rearranging yields 


$$
U_{1}=-i \omega V_{0} \frac{1}{\gamma} \frac{p_{1, \text { Back }}}{p_{0}}
$$

The time-averaged acoustic power transferred to the resonator by the speaker is

$$
\dot{W}_{a c}=\frac{1}{\tau} \int_{0}^{\tau}(\vec{F} \cdot \mathbf{v}) d t
$$

Rewriting Equation (68) in complex notation as the product of oscillatory pressure in the resonator and the velocity of the speaker diaphragm yields

$$
\dot{W}_{a c}=\frac{1}{2} \operatorname{Re}\left[p_{1, \text { Front }} \tilde{U}_{1}\right]
$$

The complex conjugate of $U_{1}$ substituted into Equation (69) produces a relation for the acoustic power transferred between the speaker and the resonator in terms of measured quantities:

$$
\dot{W}_{a c}=-\omega \frac{V_{0}}{2} \frac{1}{\gamma} \frac{1}{p_{0}} \operatorname{Im}\left[p_{1, \text { Front }} \tilde{p}_{1, B a c k}\right]
$$


Calculating acoustic power delivered to the resonator using Equation (70) requires knowledge of the volume behind the loudspeaker diaphragm. This volume was estimated using fabrication drawings of the loudspeaker housing, measuring the loudspeaker frame's volumetric displacement in water, and accounting for the volume in the enclosure taken by cooling lines and other irregularities. The volume behind each loudspeaker diaphragm was estimated to be $7.90 \times 10^{-3} \mathrm{~m}^{3} \pm 4 \%$. The uncertainty interval was estimated using the method outlined in Kline and McClintock (1953). This method was also used to estimate the total uncertainty interval for acoustic power supplied to the resonator. The power delivered to the resonator by a loudspeaker was typically $27.0 \mathrm{~W}$ $\pm 8.8 \%$ when the pressure amplitude was $\left|p_{1}\right| / p_{0}=0.0200$. Measured values for loudspeaker electrical and acoustic performance are included in Appendix B.

\section{$\underline{\text { Resonator }}$}

Figure 11 also provides a 1:10 scale view of the resonator's inner shape. The resonator was a torus formed from pipe, elbows, tees, caps, flanges, plus several custommade fittings; all from polyvinyl chloride. The resonator consisted of an upper section; on either side, a loudspeaker pod and stack assembly; and a lower section. The standing wave's pressure nodes (marked " $N$ " in Figure 11) divided the resonator into left and right symmetric halves. Each side of the refrigerator comprised half of the upper resonator section, two loudspeaker pods, a stack assembly, and half of the lower resonator section. 
Flanges on the upper resonator section were placed atop the loudspeaker pods. Bolts held rubber face gaskets between the pods and the flanges. Vertically-oriented straight pipe sections linked the flanges on either side to $90^{\circ}$ elbows. Custom-made conical diffusers, $15.3 \mathrm{~cm}$ inside diameter by $36.8 \mathrm{~cm}$ long by $10.2 \mathrm{~cm}$ inside diameter were attached at the other end of the elbows forming two horizontal sections that were linked by a $10.2 \mathrm{~cm}$ inside diameter tee at the upper pressure node. The base of the tee was connected to the flow delivery system at " $U$ " in Figure 11. The arrows marked " $U$ " and " $L$ " in Figure 11 show the two possible flow directions through the resonator. The darkened arrow gives the flow direction when the device operates as an open-cycle thermoacoustic refrigerator and the open arrow the flow direction when the device operates as an open-cycle thermoacoustic heater. The lower resonator section resembled the upper resonator section, but flipped vertically to join with the bottom of the stack assemblies. The lower section's tee was connected to the flow delivery system at " $L$ " in Figure 11. Threaded rods were used with bolts to attach the stack assemblies and the lower resonator assembly to the bottom of each speaker pod.

The conical diffusers were installed to reduce the importance of non-linear effects in the refrigerator. If a standing wave with frequency $f$ exists in a cavity of uniform cross section, it may also co-exist with harmonics of frequency $n f$ where $n$ is an integer. High-amplitude harmonics can absorb significant acoustic power from the fundamental. Harmonic amplitudes can be reduced by careful choice of resonator geometry. With a resonator having a non-uniform cross section, the wavelength for each mode shifts 
because the stiffness and inertia that each waveform experiences is different. In such a resonator, harmonic amplitudes are reduced because harmonic frequencies do not coincide with resonance frequencies.

The stack and heat exchangers do not greatly alter the stiffness experienced by each acoustic mode because they are fairly short and acoustically somewhat transparent. Large changes in resonator shape are needed to produce a significant change in mode frequencies. A non-uniform cross section was produced by placing the conical diffusers on either side of the symmetric axis. These conical diffusers had a $7^{\circ}$ included angle to minimize streaming of the oscillatory flow from the inner wall of the diffuser. Acoustic streaming is a steady flow component that is induced by the oscillating flow. Streaming is significant at acoustic amplitudes large enough for the resonating gas to depart from sinusoidal paths $\left(\left|p_{1}\right| / p_{0} \sim 0.1\right)$. Streaming velocity is proportional to the square of pressure amplitude.

Harmonic content in a resonator can be estimated by solving the wave equation in pressure and volumetric velocity. To accomplish this without resorting to numerical integration, the acoustic behavior in the conical diffuser can be approximated by assuming a step change in cross-sectional area located at its center of volume. Pressure amplitudes match on either side of the step change in cross sectional area as do the volumetric velocities.

Consider the simple quarter-wavelength resonators shown in Figure 12. Figure 12(a) shows a resonator having two cross sectional areas connected by a conical diffuser. 


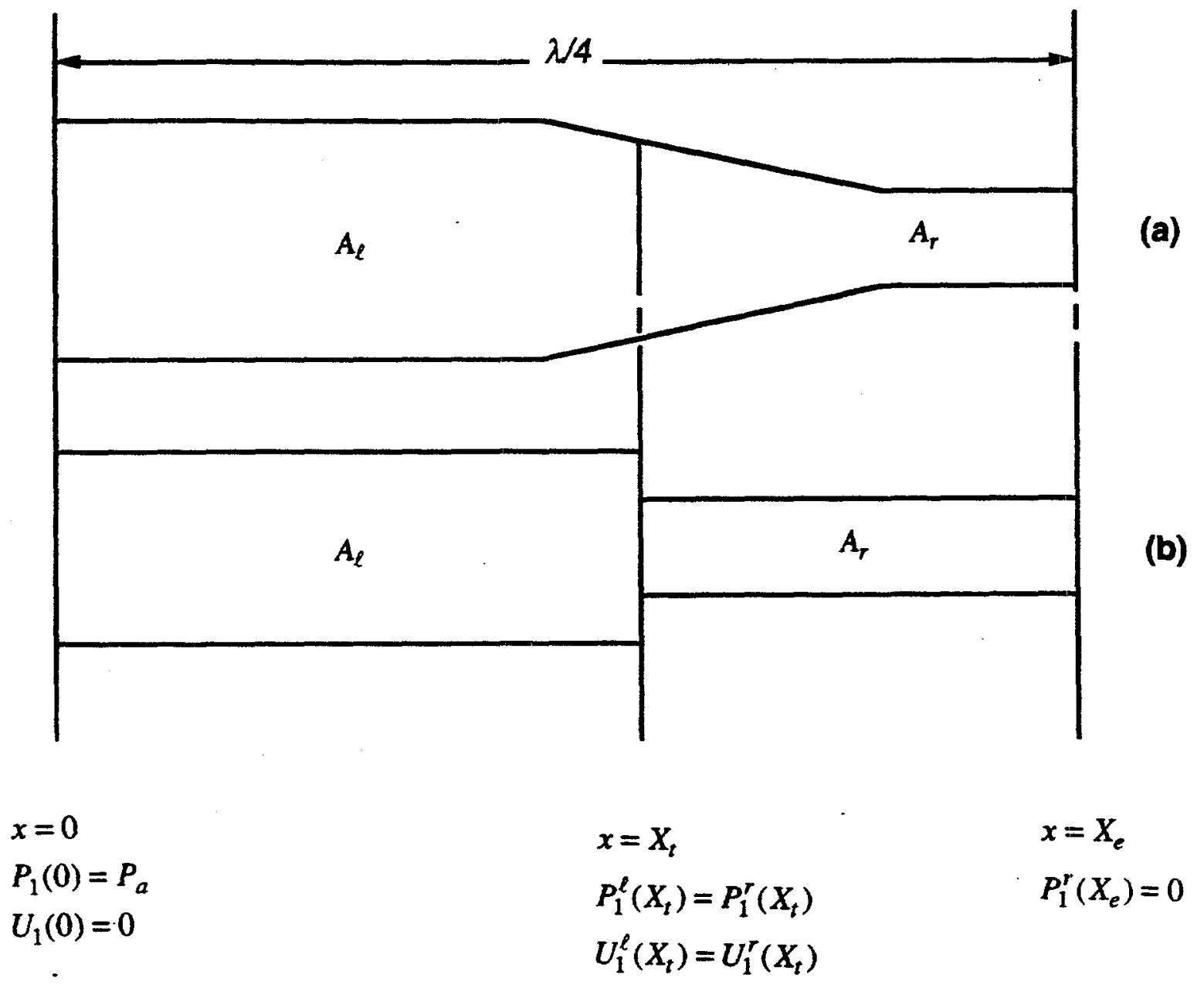

Figure 12. Domain and key variables for (a) resonator with a diffuser and (b) its mathematical representation. 
Figure 12(b) is its approximate mathematical representation. The left part of the resonator has a uniform cross sectional area, $A_{\ell}$, starting at $X=0$. This section continues until a transition in cross sectional area is made to $A_{r}$ centered about $X=X_{t}$. The second section transitions to a uniform cross section, $A_{r}$ up to $X=X_{e}$. Two sets of plane-wave solutions are possible for these resonators. One family of solutions has a pressure anti-node at $X=0$. Substituting the boundary conditions mentioned above into the wave equations for pressure and volumetric velocity yields

$$
\frac{A_{r}}{A_{\ell}} \cot \left(k_{n} X_{t}\right)+\tan \left(k_{n} X_{e}-k_{n} X_{t}\right)=0
$$

Another family of solutions has a pressure node at $X=0$. Substituting the boundary conditions into the wave equations for pressure and volumetric velocity yields

$$
\frac{A_{r}}{A_{\ell}} \tan \left(k_{n} X_{t}\right)+\tan \left(k_{n} X_{e}-k_{n} X_{t}\right)=0
$$

Given $A_{\ell}, A_{r}, X_{t}$, and $X_{e}$ the wave numbers of the fundamental and the harmonics, $k_{n}$, that satisfy these periodic relations can be found. If the resonator's wave number for any of the modes above the fundamental is an integer multiple of the wave number for the fundamental, then those higher modes are susceptible to excitation by the fundamental. 
The wavevector $k_{n}$ was found numerically for the first few modes of each solution. The resonance frequencies predicted by these equations were well away from any excitation frequencies. It should be noted that this procedure anticipates only the presence of modes for a given geometry, and not the amplitude of the modes.

The resonator was evacuated before being filled with the helium-argon mixture. At times, evacuation pressures below $1 \mathrm{~Pa}$ were measured at the inlet to a diffusion pump. However, the numerous fittings and the adjustments required during development of the refrigerator system made it difficult to maintain these pressures. More typical was the 13Pa level that was obtained while the diffusion pump was operating. Once the resonator was filled with helium-argon, the positive pressure of the gas minimized resonator contamination from ambient-pressure air.

Gas cylinders filled with a mixture consisting of $92 \%$ helium-8\% argon were procured commercially. In high-helium-fraction mixtures, small composition changes greatly alter the properties of the gas. To ensure homogeneity in the helium-argon mixture, each $23-\mathrm{cm}$ diameter cylinder was rolled across a 1-m distance about 100 times before service. The fundamental frequency measured in the resonator filled from the commercial gas was compared with similar measurements made with mixtures of known composition extracted from separate helium and argon cylinders. The resonance frequencies observed agreed within $\pm 0.5 \mathrm{~Hz}$, indicating that the commercial and knowncomposition helium fractions agreed within $\pm 0.2 \%$. 


\section{$\underline{\text { Refrigerator Supports }}$}

To provide thermal and acoustic isolation, the refrigerator was hung from scaffolding with dynamic climbing rope. The rope connected the scaffolding to supports on the loudspeaker pods. Tests were initially planned with a pure-helium filled resonator. Unfortunately, the loudspeakers excited a fundamental vibration mode in the refrigerator structure near the pure-helium acoustic resonance frequency. Piezoelectric accelerometers attached to the refrigerator were used to characterize these vibrations. The accelerometers detected three distinct motions at $120 \mathrm{~Hz}$ : (1) an x-direction out-of-phase oscillation between the left and right loudspeaker pods, (2) an x-y plane rocking motion, (3) an apparent torsion between the left and right pods about the $\mathrm{X}$ axis.

The unbalanced reciprocating motion of the loudspeakers drove vibrations in the $\pm x$ direction and excited a low-Q mechanical resonance peak centered near $153 \mathrm{~Hz}$. These vibrations were not only a nuisance, they were also potential conduits for acoustic power dissipation from the system. After some trials, use of a $92 \%$ helium- $8 \%$ argon gas mixture was found satisfactory to keep the acoustic resonance $(94 \mathrm{~Hz})$ away from the structural resonance frequency. While resorting to the helium-argon mixture reduced the power density of the refrigerator (proportional to the product of mean pressure and sound speed), increases in refrigerator mean pressure were made to compensate. By increasing the mean pressure, the helium-argon filled resonator operated within $10 \%$ of the initially planned design point with pure helium. An added benefit of this change was slightly better mechanical-to-acoustic efficiency. The $94-\mathrm{Hz}$ operating frequency placed the 
system nearer the $\sim 65-\mathrm{Hz}$ mechanical resonance frequency of the loudspeakers' suspension systems.

\section{Insulation}

Insulation was required to reduce the thermal coupling between the refrigerator and its surroundings. The entire resonator and speaker pods were contained in a large box filled with 10-mm diameter polystyrene packing beads. Instrument cables, power leads, and water lines were fed through the open top of the box. The efficacy of this insulating method was later revealed in a comparison of the system electrical power dissipation with the thermal power rejected through the water cooling lines and applied flow circuit. The refrigerator was allowed to operate at $\left|p_{1}\right| / p_{0}=0.0200$ for enough time (typically 6 hours) to reach a quasi-equilibrium state. Thermal equilibrium was defined to be a state when the discrepancy between the electrical power supplied to the refrigerator and the power removed by the water and applied flow cooling systems fell below a few percent. The discrepancy for the overall refrigerator was defined as

$$
\Delta \%=100 \cdot\left[\psi+\sum \dot{Q}_{\text {water }}\right] / \psi
$$

where $\psi=\sum \dot{Q}_{\text {elec }}-\sum \dot{W}_{\text {elec }}+2 \Delta \dot{H}_{0}$ and $\Delta \dot{H}_{0}=\left(p_{0} / T_{0}\right)(\gamma / \gamma-1) U_{a}\left(T_{U}-T_{L}\right)$. Soon after the loudspeakers were started and the gas in the resonator brought to $\left|p_{1}\right| / p_{0}=0.0200$, the electrical power supplied to the refrigerator usually exceeded the power removed by the 
water and applied flow distribution systems by $20 \%$. This discrepancy after 6 hours of operation depended somewhat on diurnal changes in water supply temperature, but was typically within $\pm 3.0 \%$.

\section{Stack Assemblies}

The stack assembly consisted of a room-temperature heat exchanger, a thermoacoustic stack, and cold heat exchanger. The stack and cold heat exchanger were contained in a section of $15.32-\mathrm{cm}$ inside diameter polyvinyl chloride pipe. Attached to one end of the pipe was a flange. To the pipe's opposite end was attached a hot heat exchanger.

Several approaches to form open channels between stack plates were tried at first. These included the use of sacrificial spacers between plates, removed by chemical or mechanical means. Finally, a loom was developed to form open channels by separating individual fiberglass sheets with threads of epoxy-coated Nylon ${ }^{\circledast}$ monofilament line. The loom consisted of an aluminum plate with a pattern of five alignment rods and 12 stringing pillars drilled into it. Numerous square fiberglass sheets, $15.24 \mathrm{~cm}$ on a side, were pre-cut. Five holes were drilled into each sheet to allow sheet alignment during fabrication and passage of the fiberglass support tubes during final assembly. A sheet was placed on the loom plate and held in place with dowel rods through the five alignment holes. The continuous epoxy-coated monofilament was then strung around the rods to form a series of near-parallel strands on the surface of the fiberglass sheet. 
A prototype set of stacks was produced by assembling 73 layers of these monofilament-separated fiberglass sheets into a block. The unsupported fiberglass sheets forming the block were then cut with a diamond saw into hemi-cylindrical sections which conformed to the inner diameter of the polyvinyl chloride pipe. The sections were then inserted within the polyvinyl chloride pipe and held in place with five fiberglass support tubes passing through the major diameter of the stack. These fiberglass tubes also fed sheathed $0.51-\mathrm{mm}$ diameter Type-K thermocouples to the center channel of the stack. These prototype stacks were then placed into the resonator and used during early tests of the refrigerator system.

An improved version of the prototype stacks with more accurate temperature sensors were later built for the final version of the refrigerator. The fiberglass procured for the improved stack was found to have higher resin content than the fiberglass in the prototype stacks. More resin made diamond saw cutting of individual sheets on layered blocks impractical, so a different fabrication procedure was developed. Well-supported sheets could be cut on a mill. However, the milling tool was unable to cut individual sheets in the pre-assembled block into hemi-cylindrical sections as before. Instead, individual sheets were pre-cut to form the hemi-cylinder. The non-uniform distribution of fiberglass sheet thickness necessitated measurement of individual plates thickness and adjustment of individual sheet widths to conform to the inside diameter of the polyvinyl chloride pipe. 
A scale drawing of the stack is shown in Figure 13. The final stack consisted of 142 parallel, $0.25-\mathrm{mm}$ thick, fiberglass plates with length $15.24 \mathrm{~cm}$. The separation between plates in the stack was $0.81 \mathrm{~mm}$, maintained by the straight lengths of epoxy-coated nylon monofilament, aligned along the length of the stack, and located on 13.5-mm centers. The separation between plates was about two thermal penetration depths of the helium-argon mixture at the operating conditions. The resulting stack formed a series of channels with a rectangular cross section $0.81-\mathrm{mm}$ high by $12.7-\mathrm{mm}$ long, except near the outer diameter of the stack. The stack plate thickness was chosen to ensure negligible plate flexure in the standing wave and to ease stack fabrication, rather than to optimize thermoacoustic performance. While the selected plate spacing produced nearly optimal power density in the gas region, the stack plate thickness was much greater than necessary, reducing the potential power density over the entire stack cross section. The stack plate thickness was enough to impose the boundary condition $T_{1}(0)=0$ at the plate surface. Fiberglass was selected to minimize heat conduction along the stack plate in the direction of sound propagation. The stack length was chosen to be much less than the radian length of the sound wave but much larger than the oscillatory gas displacements produced by the standing wave.

Stainless steel sheathed copper-constantan thermocouples with "special limits of error" and 0.51-mm diameter [10] were fed to the central stack plate through a series of five 3.2-mm outside diameter fiberglass tubes (marked "LOI" through " $L 10$ " and "ROI" 

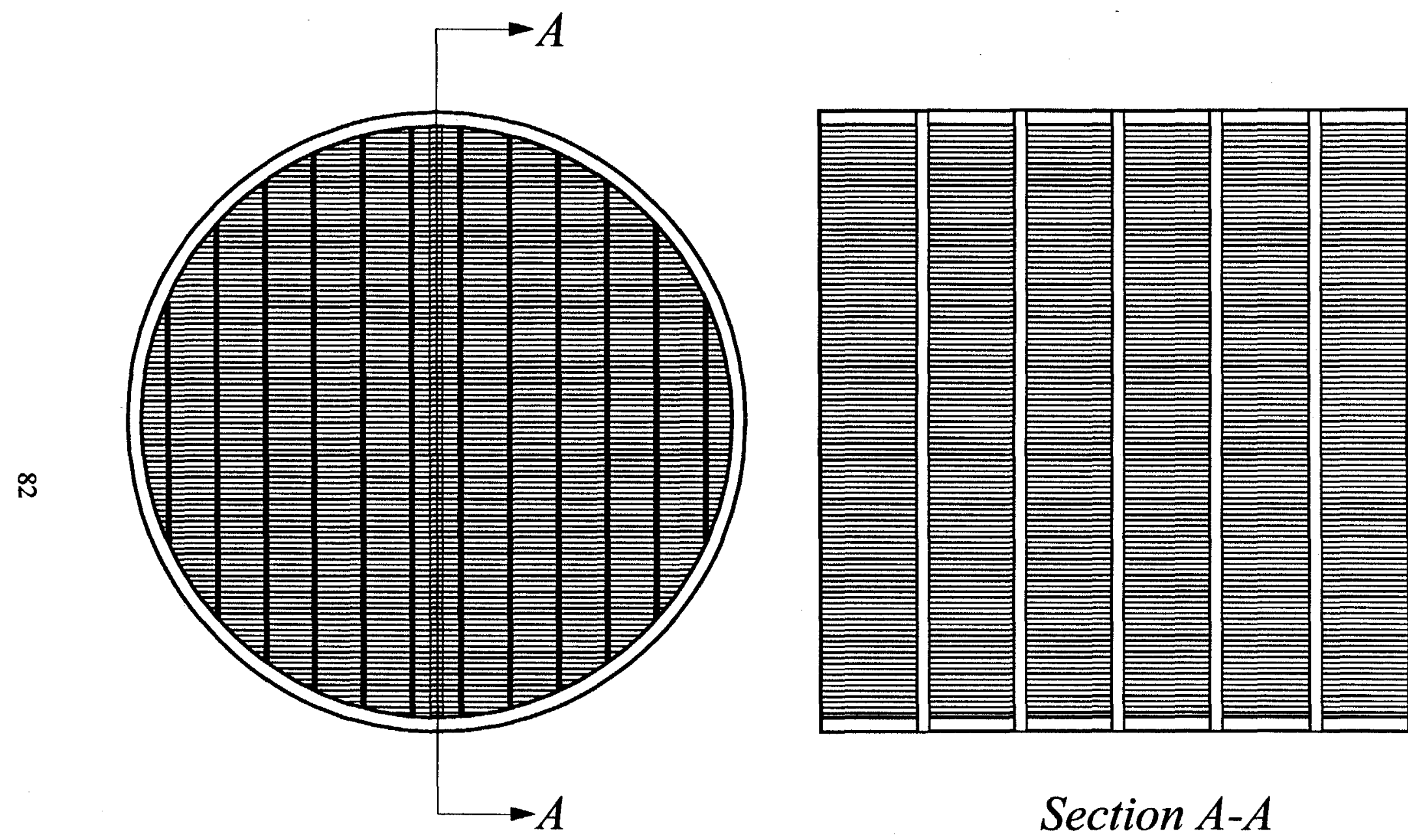

Section $A-A$

Figure 13. Scale drawing of stack showing end and lateral views through the central cross section. 
through " $R 10$ " on Figure 17). The junction of each thermocouple was glued to the stack surface with epoxy at 10 equally spaced axial positions in a rectangular channel located nearest the center of the stack's major diameter. The thermocouples were attached to the plate $3 \mathrm{~mm}$ to one side of the fiberglass tubes. The rectangular channel containing the thermocouples was blocked at each end to eliminate acoustic power dissipation on the thermocouple wires contained in that channel. The temperature measured was produced primarily by thermoacoustic heat pumping on the solid surface in the adjacent unblocked channels.

\section{Hot Heat Exchanger}

Adjacent to and above each stack was a hot heat exchanger used to transfer heat from the refrigerator to the environment. The gap between the hot heat exchanger and the stack was minimized so that the oscillating gas was in contact with both during operation. Orthogonal views of the hot heat exchanger are depicted in Figure 14. This exchanger spanned $1.27 \mathrm{~cm}$ of resonator length. A rubber $\mathrm{O}$-ring sealed the hot heat exchanger brass case to the stack assembly on one face and a polyvinyl chloride pipe on the other face. The exchanger's single bank of eight $0.48 \mathrm{~cm}$ outside diameter copper tubes were softsoldered into its $14.8-\mathrm{cm}$ inside diameter brass case and to its 80 copper fins, of $0.53-\mathrm{mm}$ thickness, and 1.27-mm separation. A copper-constantan thermocouple measured the temperature of one of the copper fins. A total of $90 \mathrm{~cm}^{2}$ (52\% of the case inside area) was 


\section{Central Cross Section}

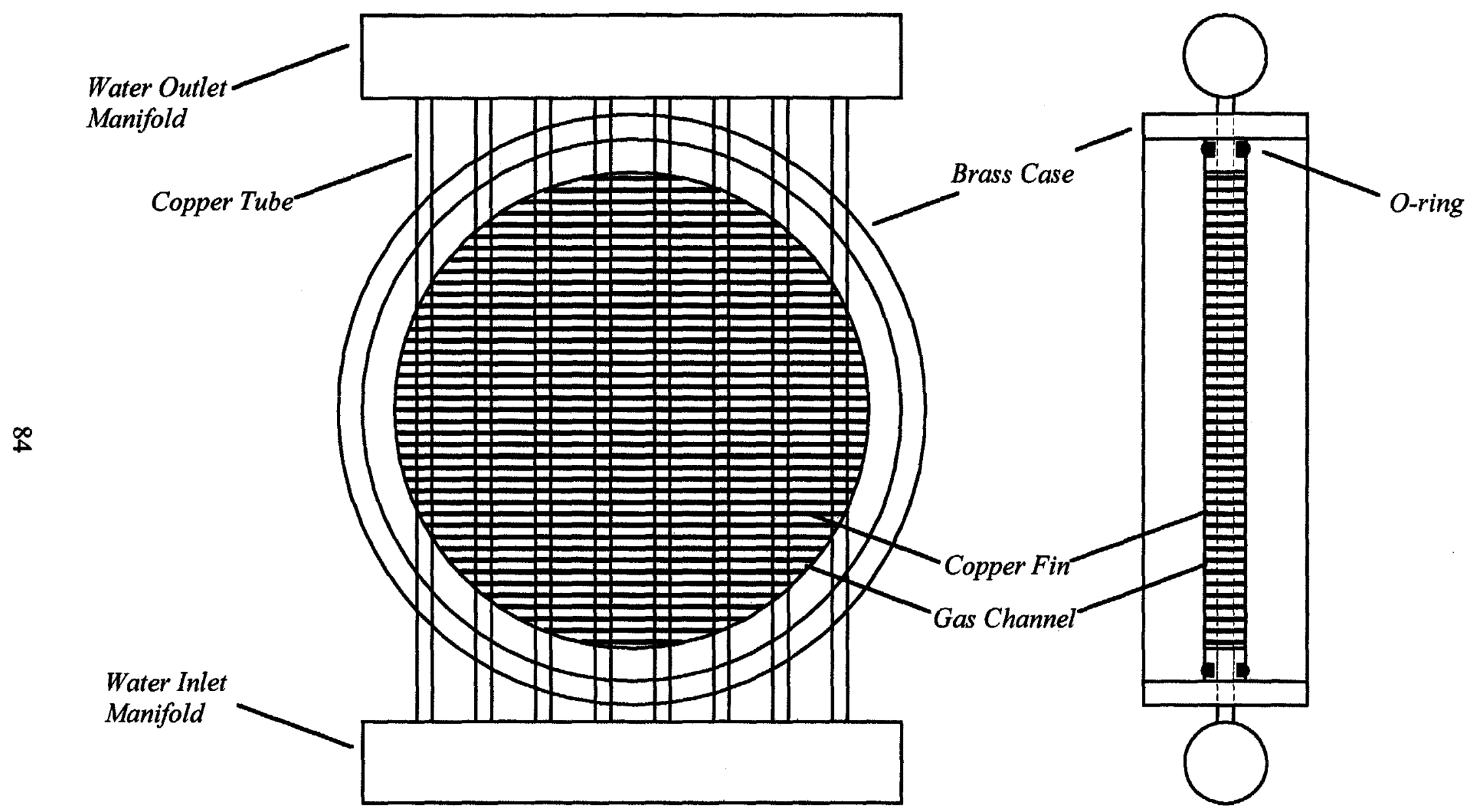

Figure 14. End and lateral views of hot heat exchanger. All dimensions are to scale, except that the fin spacing has been exaggerated by a factor of two for clarity. 
open to gas. Manifolds connected the tubes at the inlet and outlet of the exchanger. A copper-constantan thermocouple placed inside each manifold measured the water temperature entering or leaving the exchanger (marked " $L P$ ", " $L O$ ", and " $R P$ ", " $R O$ " on Figure 17). The water flow rate to the exchanger was measured with a variable area rotameter. A valve attached to the rotameter controlled the water flow rate to the heat exchanger.

The heat rejection rate from the exchanger was calculated from the flow rate and manifold temperature measurements. This calculation was then corrected to determine the thermoacoustic contribution to the heat rejection rate at the hot end of the stack in the presence of applied flow. A control volume was drawn about the heat exchanger. A balance was made between the thermoacoustic energy flux rejected at the hot heat exchanger, the flow enthalpy through the control volume and the heat exchanger heat removal. This balance for an ideal gas yields the rejected thermoacoustic energy flux:

$$
\left(T_{0} \rho_{0} \overline{s_{1} u_{1}}\right)_{t c 1}=\dot{Q}_{h x}+\left(\overline{p_{1} u_{1}}\right)_{U}+\left(T_{0} \rho_{0} \overline{s_{1} u_{1}}\right)_{U}-\left(\overline{p_{1} u_{1}}\right)_{t c 1}+\frac{p_{0}}{T_{0}}\left(\frac{\gamma}{\gamma-1}\right) U_{a}\left(T_{t c 1}-T_{U}\right)
$$

where $\dot{Q}_{h x}=\dot{m} c_{p}\left(T_{o}-T_{i}\right), \quad T_{t c 1}$ is the temperature measured by the first stack thermocouple, and $T_{U}$ is temperature measured above the hot heat exchanger. The second and fourth terms on the right side of Equation (74) are equal, and the third term on the right side of Equation (74) is zero. Uncertainty in the calculated heat rejection rate was 
typically less than $\pm 8 \%$ of reading, but was a strong function of applied flow rate and water flow rate through the heat exchanger. At $50 \mathrm{~g} / \mathrm{s}$ water flow rate, the temperature rise as the water passed through the heat exchanger was $4.8^{\circ} \mathrm{C} / \mathrm{kW}$. The thermal resistance measured between the copper tubes and the water flowing through them was $77^{\circ} \mathrm{C} / \mathrm{kW}$. The thermal resistance from the middle of each fin to its nearby tubes was calculated to be $3.4^{\circ} \mathrm{C} / \mathrm{kW}$.

\section{Cold End Heaters}

A heat exchanger was placed at the cold end of each stack. The cold heat exchangers allowed versatile measurements and control in these experiments, but they were unnecessary for cooling the steady flow of gas itself. They also served to reduce the transient time to equilibrium after a change in applied flow rate. By holding the stack ends at constant temperature the only temperature adjustments required were at interior points. An added benefit of the cold heat exchangers was to maintain the large volume downstream from the stack at a nearly constant temperature. This minimized the shift in resonance frequency as flow conditions changed. The cold heat exchanger, depicted in

Figure 15, consisted of a continuous nichrome ribbon, $50.8-\mu \mathrm{m}$ thick by $6.35-\mathrm{mm}$ wide forming a grid. Nichrome resistivity changes are small over a wide range of temperature. The room-temperature electrical resistance of the ribbon was about $30 \Omega$. Power from voltage-controlled alternating electric current was dissipated in the nichrome ribbon. A digital multimeter detected rms-voltage across the ribbon. A thermocouple was placed 


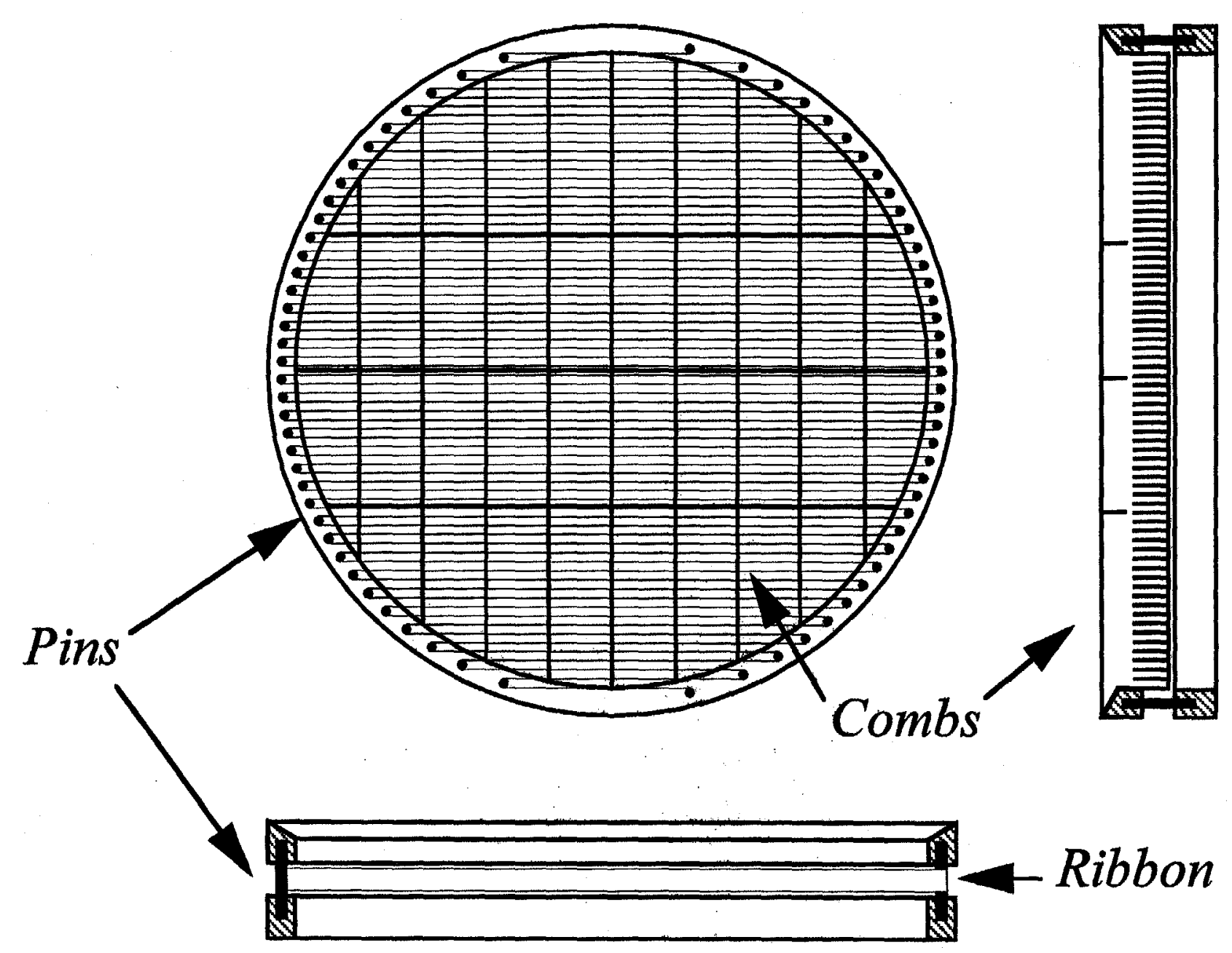

Figure 15. Scale drawing of cold heat exchanger showing an end view and two lateral cross sections. For clarity, the end view has the top section removed. 
near each heater and connected to a temperature limiting interlock that cut power to the heaters when the temperature exceeded a set point. This interlock proved particularly useful once, during a malfunction of the loudspeaker power system.

Calculation of the power absorbed by the stack from the cold end heater required some compensation. In the presence of applied flow, some energy was transferred by convection between the cold end heater and the applied flow. The cold end heat absorption rate was corrected for the convection component by measuring the temperatures on either side of the heat exchanger and using the relation for an ideal gas

$$
\left(T_{0} \rho_{0} \overline{s_{1} u_{1}}\right)_{t c 10}=\dot{Q}_{c x}+\left(\overline{p_{1} u_{1}}\right)_{L}+\left(T_{0} \rho_{0} \overline{s_{1} u_{1}}\right)_{L}-\left(\overline{p_{1} u_{1}}\right)_{t c 10}+\frac{p_{0}}{T_{0}}\left(\frac{\gamma}{\gamma-1}\right) U_{a}\left(T_{t c 10}-T_{L}\right)
$$

where $\dot{Q}_{c x}=E^{2} / R_{\text {ribbon }}, T_{t c 10}$ is the stack plate temperature adjacent to the cold end heater (marked " $L 10 "$ and "R10" on Figure 17), and $T_{L}$ is the gas temperature is the lower portion of the resonator (marked " $L L "$ and " $R L "$ on Figure 17). The other terms reduce in a similar manner to analogous terms in Equation (74). The uncertainty interval associated with the measurement of $\left(T_{0} \rho_{0} \overline{s_{1} u_{1}}\right)_{t c 10}$ was estimated to be $\pm 4.0 \%$ of reading.

\section{Applied Flow Delivery}

A two-stage compressor circulated the steady flow of the helium-argon mixture through the resonator. Figure 16 shows a schematic representation of the flow control, 


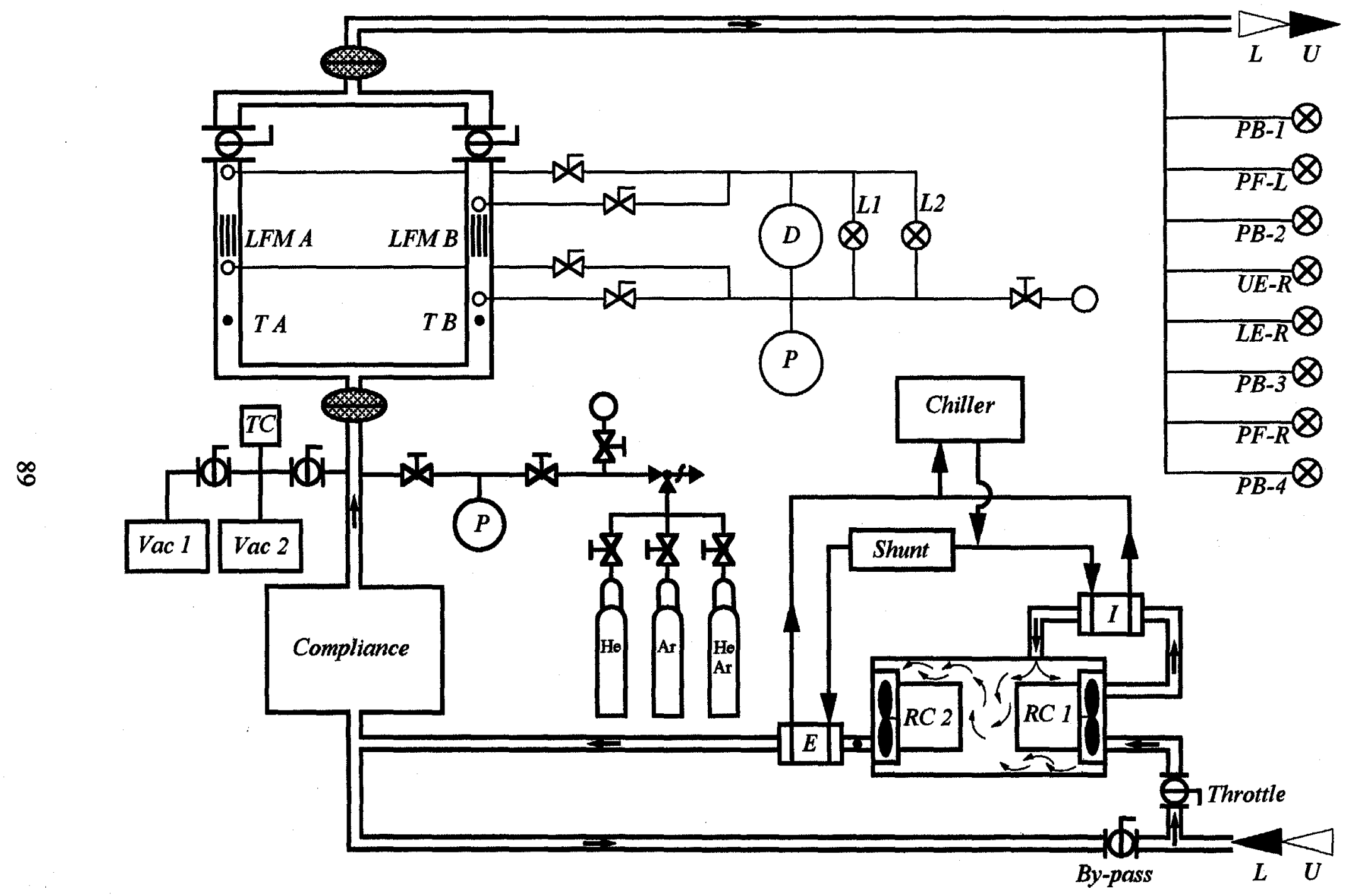

Figure 16. Schematic showing applied flow control, delivery, and measurement systems. 
delivery, and measurement system used during helium-argon mixture tests. Vinyl vacuum hose, 2.54-cm inside diameter, reinforced with wire, connected the resonator to the flow delivery system. The arrows (marked " $U$ " and " $L$ " on Figure 17) show the flow delivery paths to the resonator. The darkened arrow gives the flow path when the device operates as an open-cycle thermoacoustic refrigerator and the open arrow the flow path when the device operates as an open-cycle thermoacoustic heater. Gas was supplied from the resonator through a hose represented at the lower right corner of the diagram. Two ring compressors [11], (marked " $R C 1$ " and " $R C 2$ " on Figure 16) enclosed within a hermetically sealed cylindrical housing, provided the gas delivery to the resonator. Gas was compressed by the first stage compressor. The gas then entered a gas-to-water heat exchanger (marked " $P$ " on Figure 16) located at the exit of the first compressor stage, which provided intercooling between compression stages. The compressed and cooled gas was then routed through the cylindrical housing to cool the motor casing of each compressor. The gas then flowed through the second stage compressor exiting into the second-stage heat exchanger (marked " $E$ " on Figure 16) to remove compression heat.

Chilled water at $10^{\circ} \mathrm{C}$, supplied from a 5 -Ton refrigeration unit, removed heat from the compressor intercooler and the second stage heat exchangers. A separate loop supplied chilled water to control the temperature of a box containing the shunt resistors used to detect the electrical current supplied to the loudspeakers.

The gas flow rate through the compressor assembly was controlled by throttling flow to the suction side of the first stage compressor and by allowing some of the gas 
discharged by the second stage compressor to re-circulate to the suction side of the first stage compressor through a bypass. The gas that did not circulate through the bypass moved through an expansion chamber (marked "Compliance" on Figure 16) that acted as a low-pass acoustic filter. This compliance was added to reduce the magnitude of any low-frequency velocity oscillations generated by the compressors. Pressure oscillations were reduced by the inertance provided by lines connecting the compliance and the entrance of the resonator.

The gas continued past the vacuum and charging system. The vacuum system consisted of a roughing and diffusion pump in parallel and a thermocouple vacuum gauge. The charging system consisted of several gas bottles connected in parallel.

The gas then moved to the flow measurement system through a filter-strainer that was included to protect the flow measurement system from dust and minute particles. The gas was routed through two Meriam Instrument laminar flow meters [12] connected in parallel (marked “ $L F M$ ”). Normally, only one laminar flow meter was used to measure flow rate, while the other meter was isolated from the flow circuit with a ball valve.

A differential pressure formed across the laminar flow meter as gas flowed across its well-characterized restriction. When the flow through the restriction was laminar, the pressure drop across it was proportional to volumetric flow rate. Each meter was calibrated by the manufacturer using a NIST-traceable ANSI Z540-1 standard. Before the test series with the helium-argon mixture, the more frequently used flow meter was returned to the manufacturer for re-calibration and was found to be within tolerance. This 
meter was solely used for the results presented in Chapter 4. A Bourdon tube pressure gauge [13] (marked " $D$ " on Figure 16) sensed the differential pressure across the meter. Since accurate flow measurement was critical, two additional differential pressure measurements were made with piezoresistive pressure transducers [14] (marked " $L l$ " and “L2"). These transducers measured a differential pressure across the laminar flow meters. The signals from these transducers were fed to a signal conditioning box with output fed to a digital multimeter [15]. The piezoresistive transducer measurements supplemented (and for the test series reported in Chapter 4, replaced) the Bourdon gauge as the primary differential pressure measuring device. Before installation, the piezoresistive transducers were calibrated at a $323 \mathrm{kPa}$ case pressure using a water manometer.

The net mass flow rate supplied to the resonator was computed with volumetric flow rate, temperature (marked " $T A$ " and " $T B$ " on Figure 16) [16] and Bourdon tube pressure (marked " $P$ " on Figure 16) [17] measurements taken near the flow meter. The flow finally passed through a filter strainer before returning to the resonator. Near the resonator entrance a $0.65-\mathrm{cm}$ diameter copper tube was placed that served as a reference pressure for the dynamic pressure transducers. The volumetric flow rate at the inlet to each stack was found by correcting for absolute pressure and temperature and assuming that the mass flow rate was evenly divided between resonator sides

$$
U_{a}^{01}=\frac{1}{2} \frac{P_{0}^{P}}{P_{0}^{S-P M}} \frac{T_{0}^{01}}{T_{0}^{T A}} U_{a}^{L F M}
$$


where the superscript " 01 " denotes the conditions at the hot end of the stack.

The uncertainty associated with the measurement of volumetric flow rate had several components. The uncertainty interval from the tolerances in the laminar flow meter was estimated by the manufacturer to be $\pm 0.72 \%$ root sum square of reading [18]. The pressure transducers used to measure differential pressure had several slight nonlinearities near room temperature: a thermal sensitivity shift and hysteresis that amounts to $\pm 0.04 \%$ of full scale transducer output. The uncertainty associated with the multimeter's measurement of voltage was $\pm 0.03 \%$ of reading plus two counts [19]. There were also uncertainties from the measurement of temperature at the flow meter, $\pm 2.2^{\circ} \mathrm{C}$, [20] and in the stack, $\pm 0.5^{\circ} \mathrm{C},[21]$ as well as the uncertainty interval from the measurement of absolute pressure in the resonator $\pm 300 \mathrm{~Pa}$ [22] (marked " $S$-PM" in Figure 18). The total uncertainty associated with the measurement of volumetric flow rate was estimated to be $\pm 3.0 \%$ at the lowest volumetric flow rate and $\pm 0.94 \%$ at the highest volumetric flow rate. Side-to-side asymmetry in the mass flow rate was not accounted for in this estimate, and, in any case, was indeterminate by this measurement technique. To minimize potential side-to-side flow asymmetry, the flow impedance on each resonator side was kept small and the temperatures of surfaces on either side of the resonator were kept symmetric during measurement. 


\section{Instrumentation}

Figure 17 shows the location of the thermocouples within the resonator. Two types of thermocouples were used: Type $\mathrm{K}$ and Type $\mathrm{T}$. The temperature sampling procedure was identical for each data set. Thermal equilibrium was identified by comparing the total electrical power delivered to the refrigerator to the total thermal power removed by the water cooling circuits. Data were collected once these values agreed within $\pm 3 \%$. All temperatures were read with a thermometer with an electronically compensated ice point [23]. Data were taken serially over a 30-minute period. A stopwatch was used to minimize measurement bias by establishing a consistent data-collection time after switching between thermocouple channels. Type-K thermocouples were placed to measure the temperature of the entering and exiting flows in the straight sections near the pressure nodes (marked "TT" and "TO" on Figure 17). Another thermocouple was placed near the upper pressure node (marked " $T U$ " on Figure 17). Four movable themocouple probes $56 \mathrm{~cm}$ long were placed at the four elbows to measure the spatial temperature distribution between the elbows and the stack assembly (marked " $L U$ ", " $L L$ ", " $R U$ ", and "RL"). Each movable probe consisted of a tube containing a Type-K thermocouple bent $90^{\circ}$ to place the thermocouple junction perpendicular to the oscillatory flow. This orientation minimized measurement error from thermoacoustic heat pumping on the surface of the tube itself near the thermocouple wire junction. Two additional thermocouples were placed in the lower right elbow to measure the temperature of the 


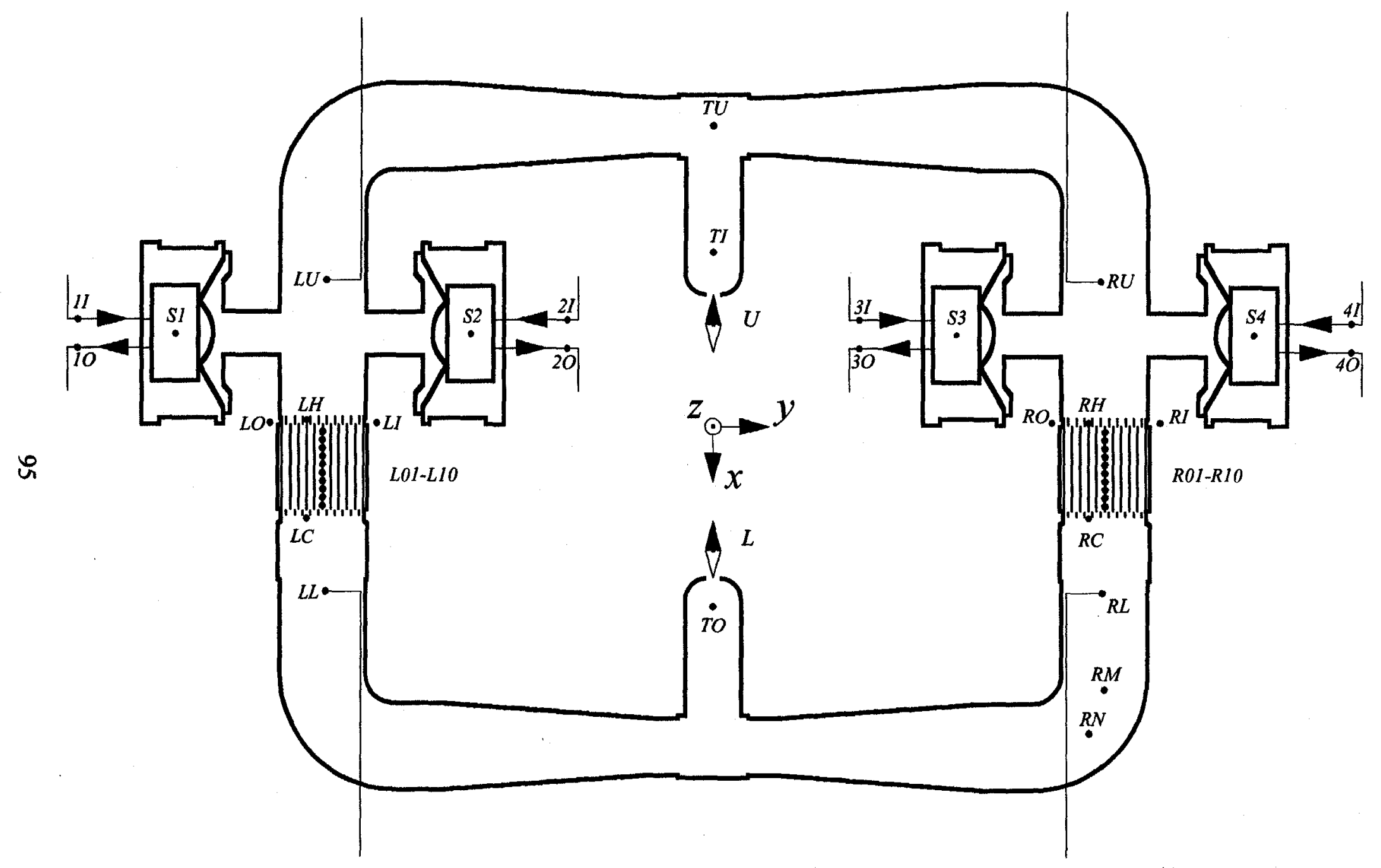

Figure 17. Temperature sensor placement. 
flow exiting the stack in refrigerator mode (marked " $R M$ " and " $R N$ " on Figure 17). Each speaker was equipped with a thermocouple to measure the temperature of the pole magnet (marked "SI" through "S4" on Figure 17). Thermocouples were also placed to measure the entering and exiting water temperatures in the lines supplying water to cool the speaker magnets (marked " $1 I "--4 P$ " and " $1 O$ "- " $4 O$ " on Figure 17).

The thermocouples in and around the stack assemblies were all Type $\mathrm{T}$ with special limits of error [24]. Thermocouples were placed to measure the entering and exiting water temperatures in the lines supplying water to cool the hot heat exchangers (marked " $L F$ ", " $L O$ ", " $R \Gamma$ ", and " $R O$ " on Figure 17). A thermocouple was attached to one of the hot heat exchanger plates on each side (marked " $L H$ " and " $R H$ " on Figure 17). A thermocouple was also attached to one of the combs on each cold heat exchanger (marked " $L C$ " and " $R C$ " on Figure 17).

Figure 18 shows the location of the sensors used to measure dynamic and static pressure within the resonator [25]. Sensors measuring dynamic pressure were placed at each elbow and in front (marked "PF-L" and "PF-R" on Figure 18) and behind (marked "PB-I" through " $P B-4$ " on Figure 18) each loudspeaker diaphragm. Front-mounting threads allow each sensor to be attached from the outside diameter of the polyvinyl chloride pipe. The sensor's reference tube was connected through a 1.59-mm-capillary line to the reference manifold located near the upper pressure node. Before use, the factory calibration curves for each sensor were confirmed by comparing each sensor's 


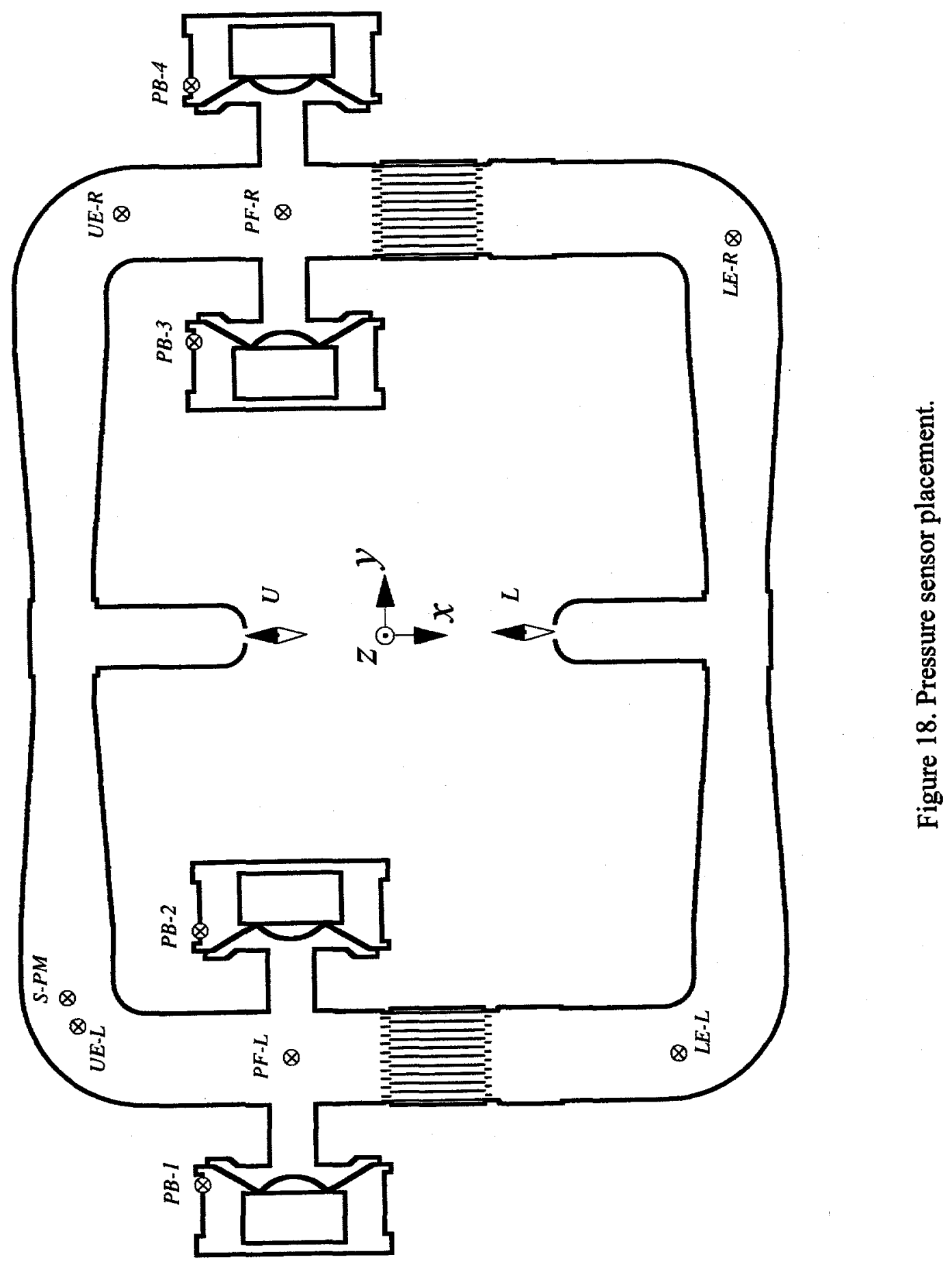


signal response to a series of known pressures. After sensor installation, in-situ calibration checks were periodically conducted by taking measurements during system pressurization. An electrical circuit provided a $10-\mathrm{V}$ dc excitation to the sensor's resistor bridge and signal conditioning. The output from this circuit was fed to a dynamic signal analyzer that sensed a complex voltage proportional to and in phase with the oscillatory pressure. To diagnose and eliminate ground-loop errors in the pressure measurement system the signal analyzer readings were occasionally compared to the readings from a lock-in amplifier [26]. The discrepancy in pressure amplitude measurements between the signal analyzer and the lock-in amplifier was typically less than $\pm 0.5 \%$ of reading. A transducer (marked " $S-P M$ " on Figure 18) was placed on the upper left elbow of the resonator to measure the mean pressure of the system referenced to atmospheric pressure. The transducer output was fed through a signal conditioning box and was read by a digital voltmeter [27]. Atmospheric pressure near the refrigerator was measured with a Bourdon pressure gauge referenced to vacuum [28].

Pressure sensors were also placed at each elbow (marked " $U E-L$ " through " $L E-R$ " in Figure 18) to measure the magnitude and phase of pressure, to characterize the standing wave and to verify standing-wave symmetry. The resonance frequency and $Q$ of the resonator were determined by scanning frequencies with the dynamic signal analyzer in swept-sine mode. These measurements were made at low amplitude to minimize thermoacoustically-induced temperature changes in the stack during each sweep. The magnitude and phase of pressure within the resonator was measured as a function of 
frequency. These measurements were compared to the linear acoustic model with good agreement in the resonator: the resonance frequency agreed within $1 \mathrm{~Hz}$ and $Q$ differed by a few percent. Measured $Q$ for the resonator was about 7 . Some difference between the linear acoustic model and measurement was found in the pressure phasing around the loudspeakers. This was attributed to limitations in the linear acoustic model calculation for the loudspeakers.

Water continuously flowed through the loudspeaker cooling cavities and the hot heat exchanger. A typical experiment began early in the morning with the starting of the loudspeaker system with an automatic timer. This allowed the loudspeakers and their massive aluminum housings to approach an equilibrium temperature. A few hours later, adjustments were made to mean pressure and the flow delivery system was brought to the desired flow rate. The temperature difference across the stack was then established by adjusting the water flow rate through the hot heat exchangers and the power supplied to the cold-end heaters. Periodic minor adjustments were necessary to prevent the system from drifting from the established values of $p_{0}$, resonance frequency, $\left|p_{1}\right|, T_{H}$, and $T_{C}$. These values were sensitive to changes in atmospheric pressure, cooling water source pressure and temperature, as well as electrical line voltage. Once equilibrium conditions were established by the heat balance between the electrical dissipation and the thermalenergy removal rate, data collection began. Each data set consisted of approximately 100 separate measurements manually recorded over a 30-minute period. A summary of the reduced data sets is tabulated in Appendix B. 


\section{CHAPTER 4}

\section{RESULTS AND DISCUSSION}

The effect of applied flow rate on stack performance was demonstrated through a series of controlled tests using $92 \%$ helium $8 \%$ argon mixture as the thermoacoustic working fluid. In these tests, the stacks were configured as either the Device (A) opencycle thermoacoustic refrigerator or the Device (B) open-cycle thermoacoustic heater shown in Figure 5. The values of $\left|p_{1}\right| / p_{0}, T_{H}$, and $T_{C}$ were held constant by adjusting the water flow rate through the hot heat exchanger, the loudspeaker power, and the electric power $\dot{Q}_{\text {elec }}$ supplied to the stack from the cold heat exchanger. This resulted in a condition that kept resonator geometry, fluid properties, $p_{1}, p_{0}, T_{H}$, and $T_{C}$ as fixed values. The resonator geometry and working fluid selection determined the resonance frequency $f$. The effect of $U_{a}$ on the dependent variables $T_{0}(x), \dot{Q}_{c}, \dot{Q}_{H}$, and $\dot{W}_{a c}$ was then measured. A narrow $T_{H}-T_{C}$ was selected for these tests to allow $\dot{Q}_{C}$ to be varied over a range of applied flow rate. Only $T_{0}(x), \dot{Q}_{c}$, and $\dot{Q}_{H}$ significantly changed with $U_{a}$. 
The pressure amplitude was kept at $\left|p_{1}\right| / p_{0}=0.0200$ to allow the results to be predictable by Rott's linear acoustic theory as amplified in Appendix A. Refrigerator operation at considerably higher amplitude was possible; however, non-linearity would have been introduced into the system from streaming, turbulence, and curvature along adiabats. Instead, a measurable power density was obtained by working-fluid selection and operation at high enough mean pressure.

As $U_{a}$ was increased, $T_{0}(x)$ along the stack was distorted from a near linear profile to a profile with exponential curvature. When $U_{a}$ was made increasingly positive, it became necessary to reduce $\dot{Q}_{C}$ to keep $T_{H}-T_{C}$ constant. During this transition toward higher $U_{a}$, there developed a functional relation between $\dot{Q}_{c}$ and $\Delta \dot{H}_{0}$ which produced a change in total cooling power $\left(\dot{Q}_{C}+\Delta \dot{H}_{0}\right)$ with $U_{a}$. When the device operated as an open-cycle thermoacoustic heater, it became necessary to increase $\dot{Q}_{C}$ to keep $T_{H}-T_{C}$ constant.

Figure 19 is a plot of acoustic power delivered to the resonator during these tests versus applied flow rate. The gray-scale line passing through the abscissa origin separates the open-cycle thermoacoustic heater data from the open-cycle thermoacoustic refrigerator data. The open circles represent the acoustic power delivered to the left resonator side found using the loudspeaker-diaphragm displacement relation given by Equation (70). The dark circles are similar values for the right side of the resonator. The amount of acoustic power delivered to the resonator was found by the diaphragm relation 


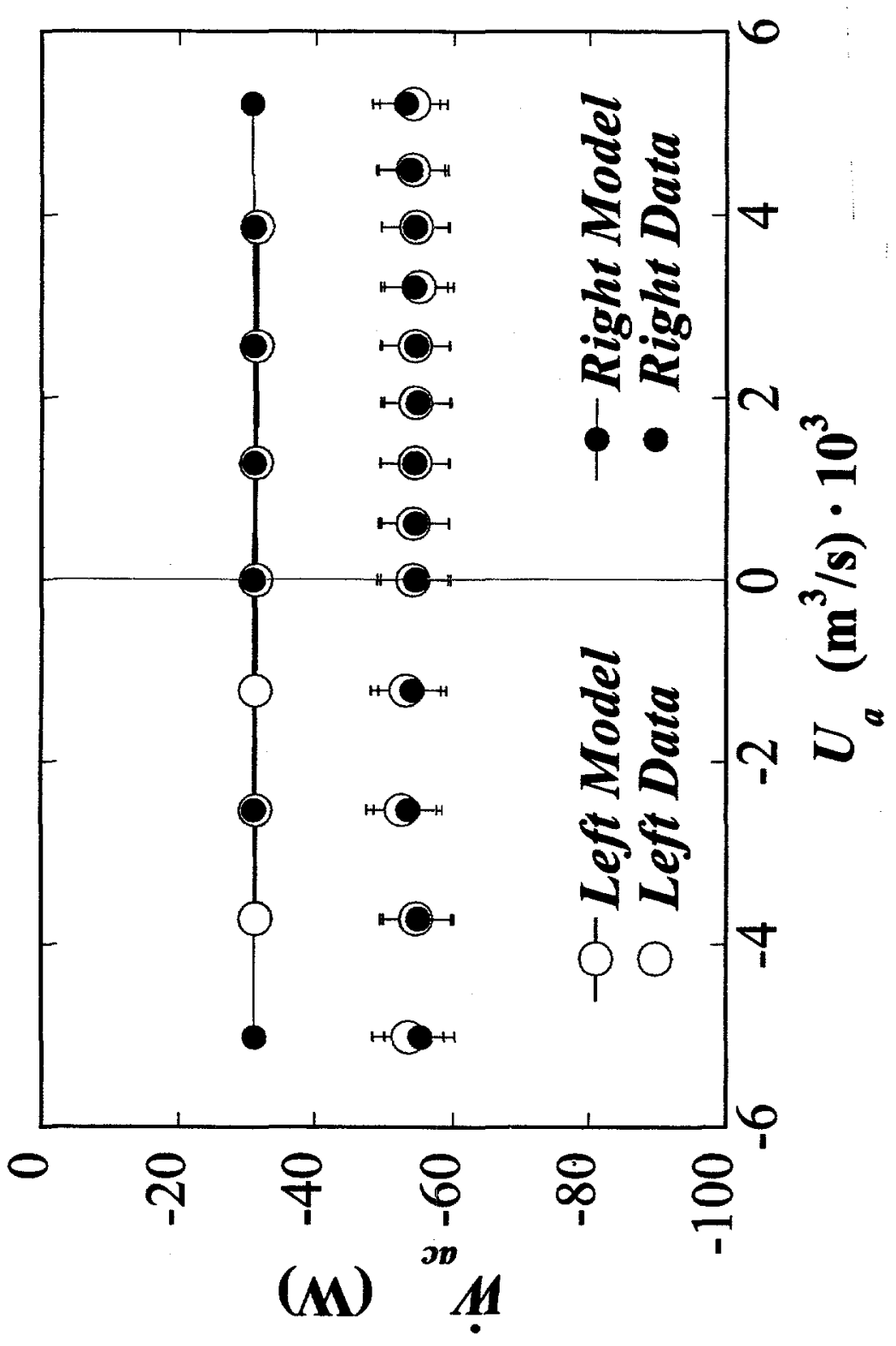

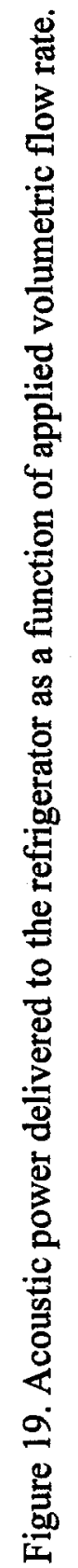


to be about $-55 \mathrm{~W}$ on a side. The uncertainty intervals shown (about $8.8 \%$ of reading) were calculated using the Kline and McClintock procedure. The comparison of measured acoustic power delivered to the left and right sides of the resonator indicates the control achieved by the microphone mixers in balancing the acoustic power delivered to each side of the resonator.

The acoustic power delivered to the resonator was independently estimated using an enclosed-loudspeaker algorithm contained within the linear acoustic model (Ward, 1997). A sample output listing of the linear acoustic model along with a brief description is given in Appendix C. Dynamic pressure around the loudspeaker is changed by the force exerted by the loudspeaker diaphragm. For a given complex pressure on either side of the loudspeaker diaphragm, the complex current and complex voltage $V_{1}$, and complex volumetric velocity in front of the speaker are related by

$$
\begin{gathered}
I_{1}=\frac{V_{1}+U_{1} B \ell / A}{R+j \omega L} \\
p_{\text {out }}=p_{\text {in }}-I_{1} B \ell / A-U_{1}\left(R_{m}+j \omega m-j k / \omega\right) / A^{2}
\end{gathered}
$$

Use of these equations requires measured or estimated values of $B \ell$ product, mechanical resistance, moving mass of the diaphragm, electrical resistance, voice-coil inductance, and diaphragm stiffness for each loudspeaker. The acoustic power delivered to the resonator can then be estimated from the relation between oscillatory pressure and 
volumetric velocity. The circles connected by lines in Figure 19 represent the values of acoustic power calculated with the linear acoustic model. The estimated acoustic power delivered to the resonator was found by these relations to be $-27 \mathrm{~W}$ on a side. To verify that the loudspeaker algorithm was correctly programmed into the acoustic model a separate hand-calculation was made, with good agreement. This estimated acoustic power exhibits the same invariance with applied flow rate as the measured data. However, the algorithm consistently under predicted the measured power value by $24 \mathrm{~W}$. Fewer assumptions are involved in the speaker-diaphragm relation given in Equation (70) than in the enclosed loudspeaker algorithm. For this reason and because the measured magnitude of loudspeaker power is greater than the algorithm value, the values measured by Equation (70) will be used in the presentation of performance index for the refrigerator system. Using the Figure 19 curves, it is a simple matter to scale the performance index values given later in this Chapter.

Regardless of the method chosen to determine loudspeaker power, Figure 19 clearly indicates that by holding the independent variables constant, the acoustic power delivered to the resonator was nearly invariant with applied flow rate. Any differences in total cooling power with applied flow rate were obtained while holding nearly constant the total acoustic power delivered to the refrigerator. 


\section{$\underline{\text { Stack Performance with Position }}$}

Figure 20 shows the right-stack temperature as a function of distance from the hot end of the stack. Profiles at three applied volumetric flow rates are shown with the temperatures at the stack ends held constant to within a few tenths of a degree Celcius. The open circles show the stack-temperature profile data with $U_{a}=5.21 \times 10^{-3} \mathrm{~m}^{3} / \mathrm{s}$ moving toward the cold end of the stack. This corresponds to the "Device A" open-cycle thermoacoustic refrigerator in Figure 5. The exponential character in the temperature profile, predicted by Equation (28), is evident in the measurements. This temperature profile is produced by the superposition of the applied flow and the second-order enthalpy fluxes similar to that described for inviscid flow by Equation (24). The temperature gradient near the hot end actually became slightly positive at this applied flow rate indicating limitation in the qualitative description provided by Equation (28). The thermocouple at $x=11.85 \mathrm{~cm}$ from the hot end of the stack read lower than would be expected by a smooth interpolation between surrounding data points.

The solid line near the open circles represents numerical results based on the linear acoustic model, described in Appendix C, and uses the relations described in Appendix A. Agreement between the measured values and the acoustic model is close near the hot end of the stack and at the stack ends. The stack end temperatures were fixed by the acoustic model itself. In the high-temperature-gradient region toward the cold end of the stack, temperatures are lower than the prediction.

The data represented by the dark squares were taken without applied flow through the 


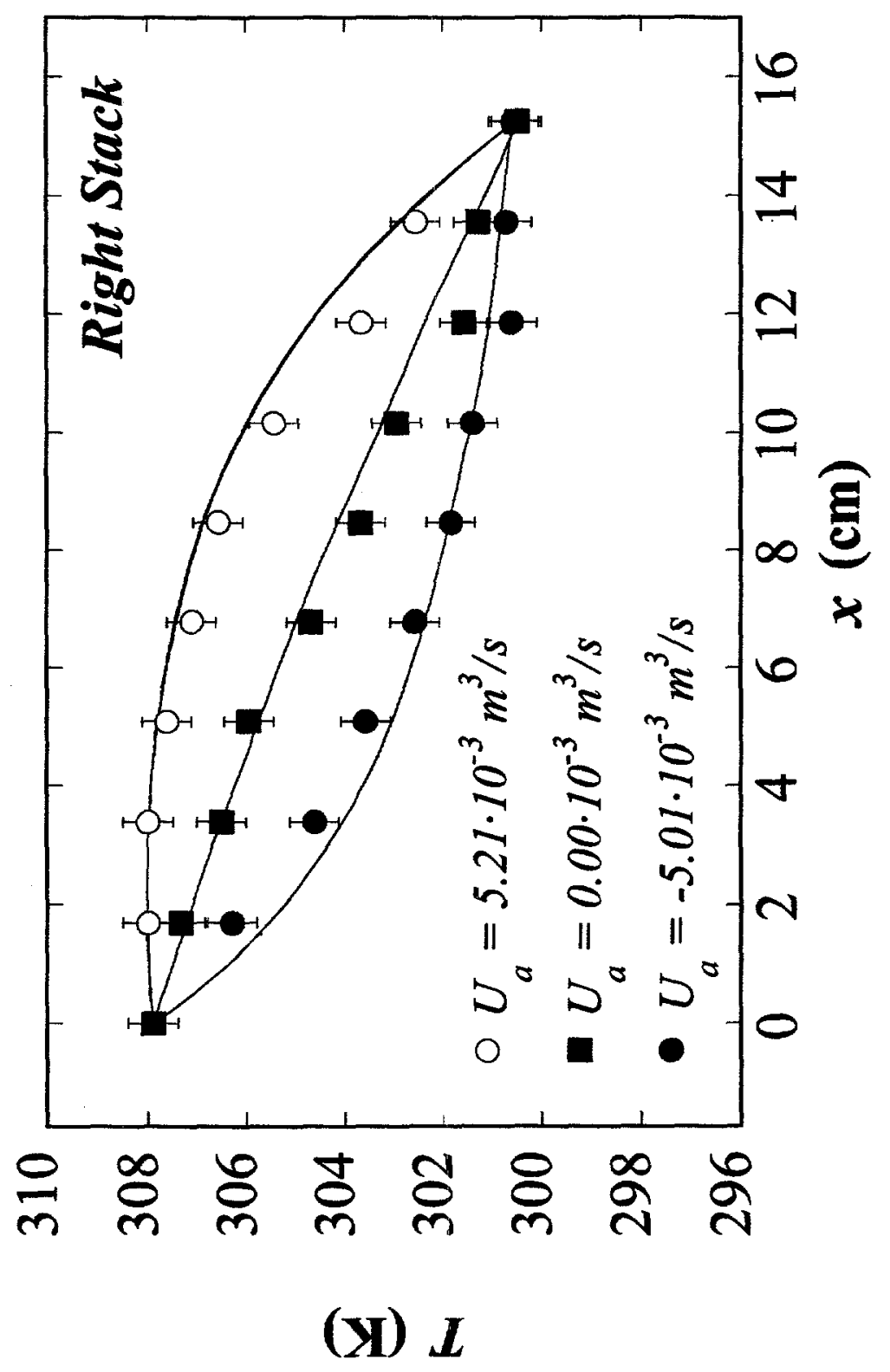

Е

芯.

은

을

호

흉

。

言吾

号

荬

西

뭉

흘

름

퐁

过

홍

寻 的

疍 岁

क

$>$

冚

昰

过

差

要

蒙总

․․ㄹ

요

응

些莫 
stack. This configuration is the closed-cycle thermoacoustic refrigerator depicted in Figure 2. The closed-cycle temperature distribution followed a nearly linear path with position. There is slight data scatter from the linear-ideal, particularly at $x=11.85 \mathrm{~cm}$. There, the reading is $0.75^{\circ} \mathrm{C}$ below a linear fit through the other data points. The solid line near the dark squares is the numerical result based on the acoustic model. This curve is contained within the uncertainty intervals for all but one data point.

The dark circles on Figure 20 are the temperatures measured as the applied flow moved toward the hot end of the stack with $U_{a}=-5.01 \times 10^{-3} \mathrm{~m}^{3} / \mathrm{s}$. This flow configuration corresponds to the "Device B" open-cycle thermoacoustic heater shown in Figure 5. The measured temperatures follow an exponential-shaped profile inverted from the open-cycle thermoacoustic refrigerator case. Unlike for the refrigerator, agreement between measured and predicted values is best toward the cold end of the stack. Discrepancy is greatest toward the hot end of the stack.

The slight discrepancy between measurement and prediction at the downstream data points for both the refrigerator and heater cases suggests a propagation effect associated with the applied flow. One possible mechanism is successive disturbances in the flow passage from the five fiberglass support tubes that pass through the major diameter of the stack. These tubes may have disturbed both the steady and oscillatory flow components introducing a cumulative flow disturbance not accounted in the acoustic model.

Besides possible flow disturbances, these supports caused a local increase in the stack surface-area to gas-volume ratio, increasing the acoustic power absorption. This effect 
would be superimposed on the flow disturbance effect. If significant, those thermocouples nearest the support tubes would be expected to read lower temperatures than expected by the acoustic model. Thermocouples located $5 \mathrm{~cm}$ and $10 \mathrm{~cm}$ from the hot end were attached in the proximity of the support tubes. While the temperature measured at $x=10 \mathrm{~cm}$ did read lower than predicted, the temperature at $x=5 \mathrm{~cm}$, in the closed-cycle case read higher. It appears that this effect was not significant.

Figure 21 is a plot of the temperature distribution along the left stack at various applied flow rates. Note that the maximum applied flow rate on this plot is $U_{a}=3.87 \times 10^{-3} \mathrm{~m}^{3} / \mathrm{s}$. Data were also taken at $U_{a}=5.21 \times 10^{-3} \mathrm{~m}^{3} / \mathrm{s}$ but to keep $T_{C}$ at the established valued would have required the removal of $\dot{Q}_{C}$ from the left-side cold heat exchanger. The use of electrical heating at the cold-end heat exchanger made heat addition possible, but precluded heat removal. The temperature versus position trend on the right stack applies equally to the left stack. The uncertainty intervals shown for each data point in Figure 20 and Figure 21 are based on standard limits of error reported by the thermocouple manufacturer [29]. On Figure 21, the measured values and the acoustic model agree within these limits for all but the thermocouple at $x=13.55 \mathrm{~cm}$. This thermocouple read consistently slightly low at all applied flow rates. Taken as a whole, the agreement between the measured and predicted temperature distributions in Figure 20 and Figure 21 is close. The small overall discrepancy appears most significant at the thermocouples positioned at $x=11.85 \mathrm{~cm}$ on the right stack and $x=13.55 \mathrm{~cm}$ 


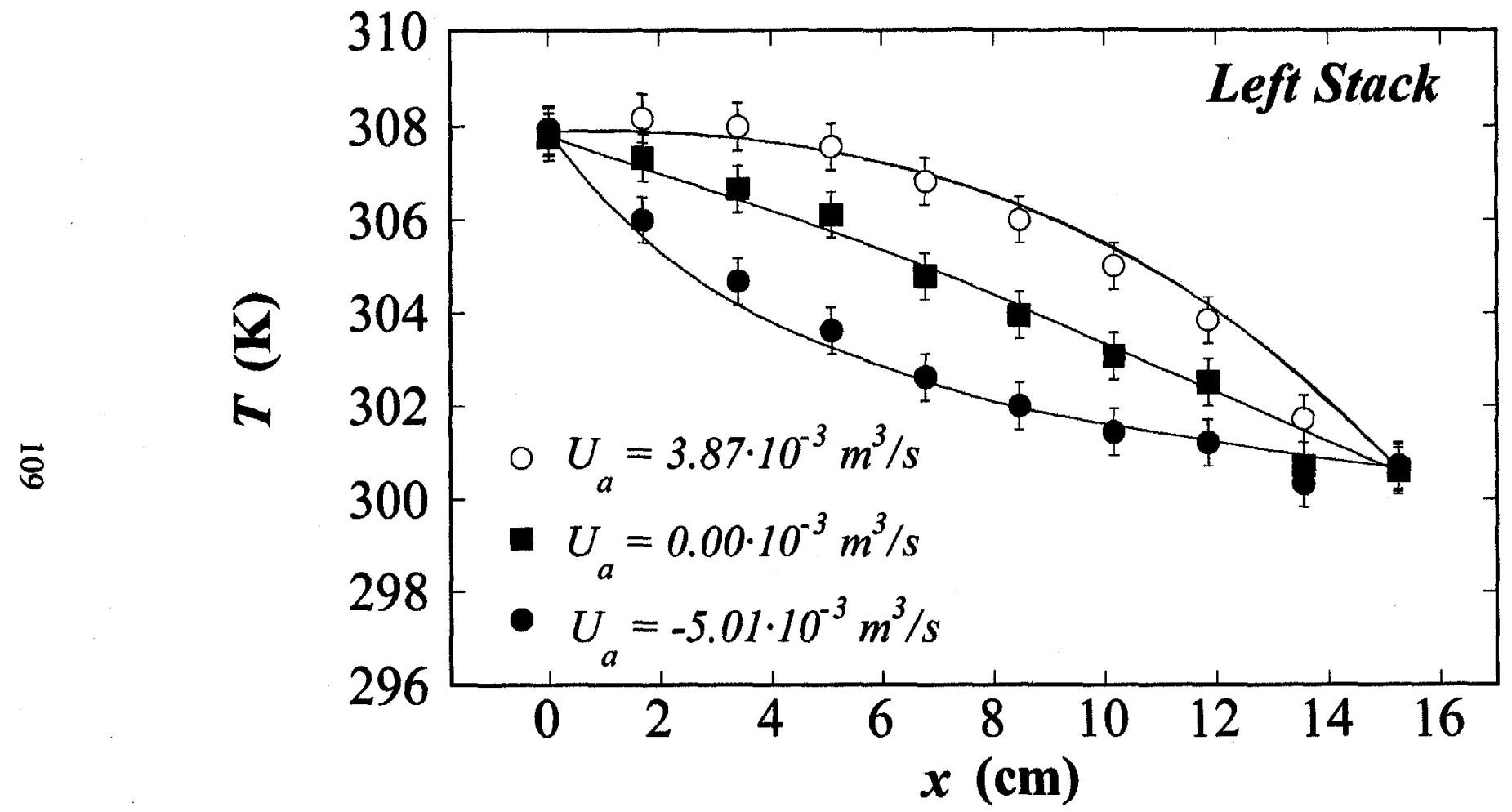

Figure 21. Left stack temperature versus distance from the hot end at various applied volumetric flow rates. The symbols are measurements. The line traces show the corresponding numerical results based on the acoustic model. 
on the left stack. A consistent departure from the predicted profile was measured at the downstream temperature locations. This departure is believed related to the cumulative effect of flow disturbances in the axial direction generated by the fiberglass stack supports and was not accounted by the acoustic model.

Non-ideal stack construction (for example, monofilament lines that are not exactly parallel to adjacent lines) appears to account for the ability of the right stack to accomodate a higher volumetric flow rate while holding $T_{H}-T_{C}$ fixed. Measurements made of the width between monofilament lines along the length of the stacks revealed some departure from parallel spacing on the right stack. The channels on the left stack were found closer to parallel. Imperfections such as these would tend to produce higher viscous dissipation and more work absorption by the right stack.

From a comparison of side-to-side differences in mean-temperature profile curvature, the magnitude of flow asymmetry is believed small. There were some other differences between the measurement and assumptions in the acoustic model calculation. Significant among these is the violation of the linear acoustic model's assumption that the gas displacement amplitude $\left|x_{1}\right|$ is much smaller than the stack length $L$ (Ward and Swift, 1997). 


\section{Stack Performance at Various Applied Flow Rates}

Figure 22 shows the right-stack heat transfer rates as a function of applied-volumetric flow rate. A gray-scale line divides the plot into four quadrants. Negative values of heat transfer rate are out of the stack system, while positive heat transfer rates are into the stack system. Positive values of $U_{a}$ correspond to operation as an open-cycle thermoacoustic refrigerator, the negative values to open-cycle thermoacoustic heater operation. At $U_{a}=0$ the device operates as a closed-cycle thermoacoustic refrigerator.

The dark triangles are cold-end-heat absorption rates, $\dot{Q}_{c}$, calculated using measured values of electric power dissipation and Equation (75). The open circles are calculated values of enthalpy change, $\Delta \dot{H}_{0}$, in the applied flow using Equation (30). The open squares represent the total refrigeration power: the sum of the cold-end heat absorption rate and the enthalpy change across the stack, $\dot{Q}_{c}+\Delta \dot{H}_{0}$. The inverted open triangles are the heat rejection rates measured at the hot end of the stack, $\dot{Q}_{H}$, described by Equation (74). The inverted dark triangles are the heat rejection rate at the hot end of the stack, $\dot{Q}_{H}$, calculated with an energy balance around the stack and using the acoustic power calculated with Equation (70). The solid lines are numerical predictions calculated using the acoustic model. A similar plot for the left stack is shown in Figure 23. As $U_{a}$ increased the total refrigeration power rose while the acoustic power needed to drive the device remained constant. This illustrates the trade-off between absorbing the entire heat load at $T_{C}$ and the absorption of enthalpy over a range of temperatures $T_{H}>T>T_{C}$. The 


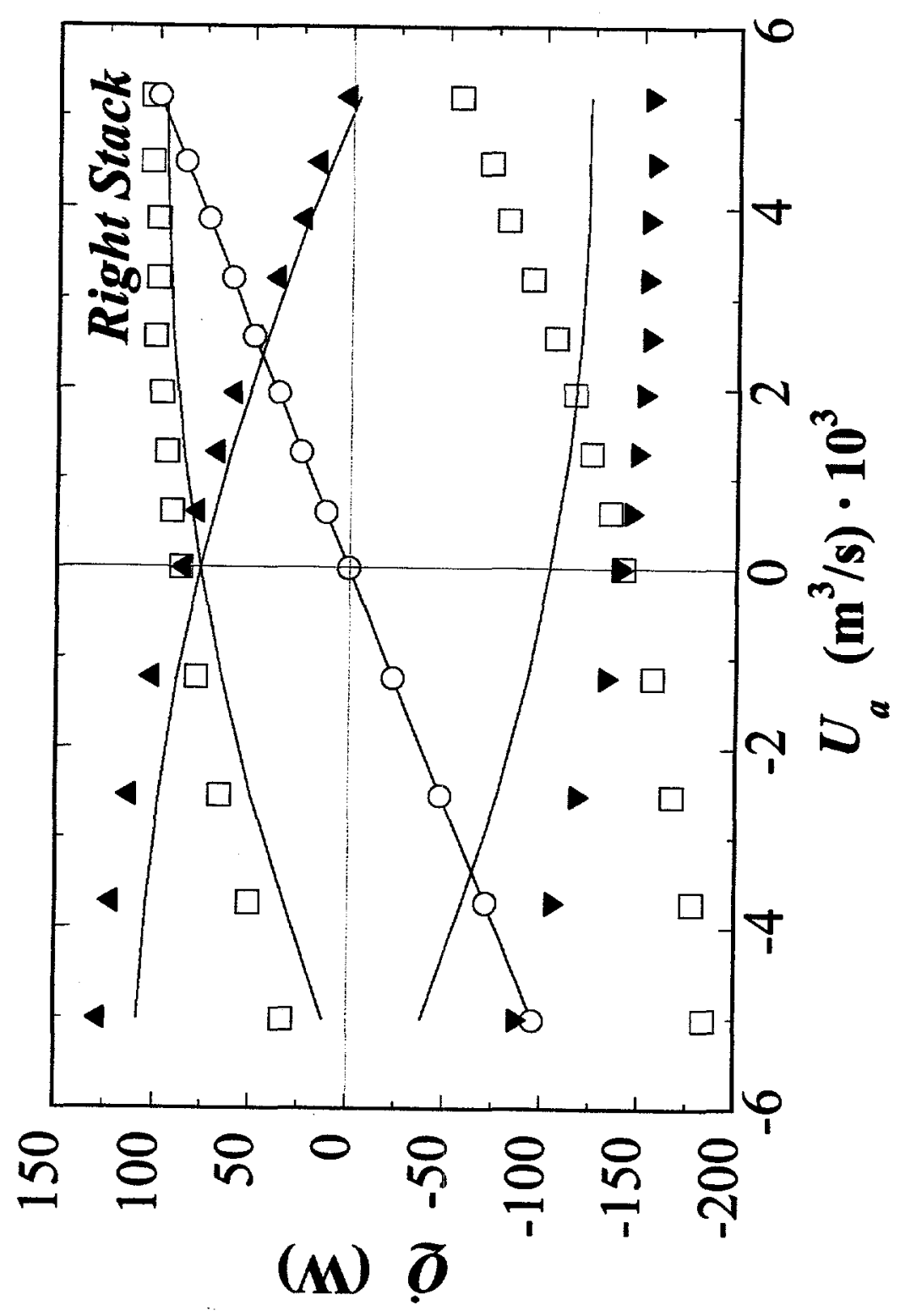

군

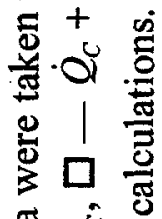

营 व

$=1$

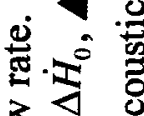

高

. 0 :

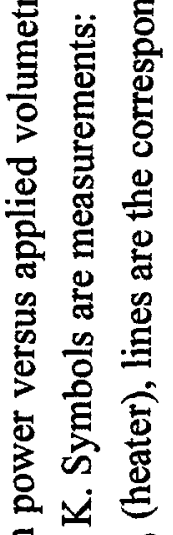

융

t)

.

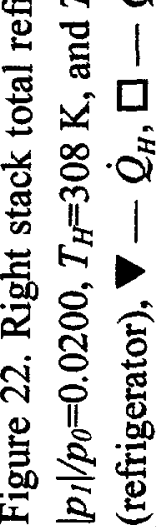




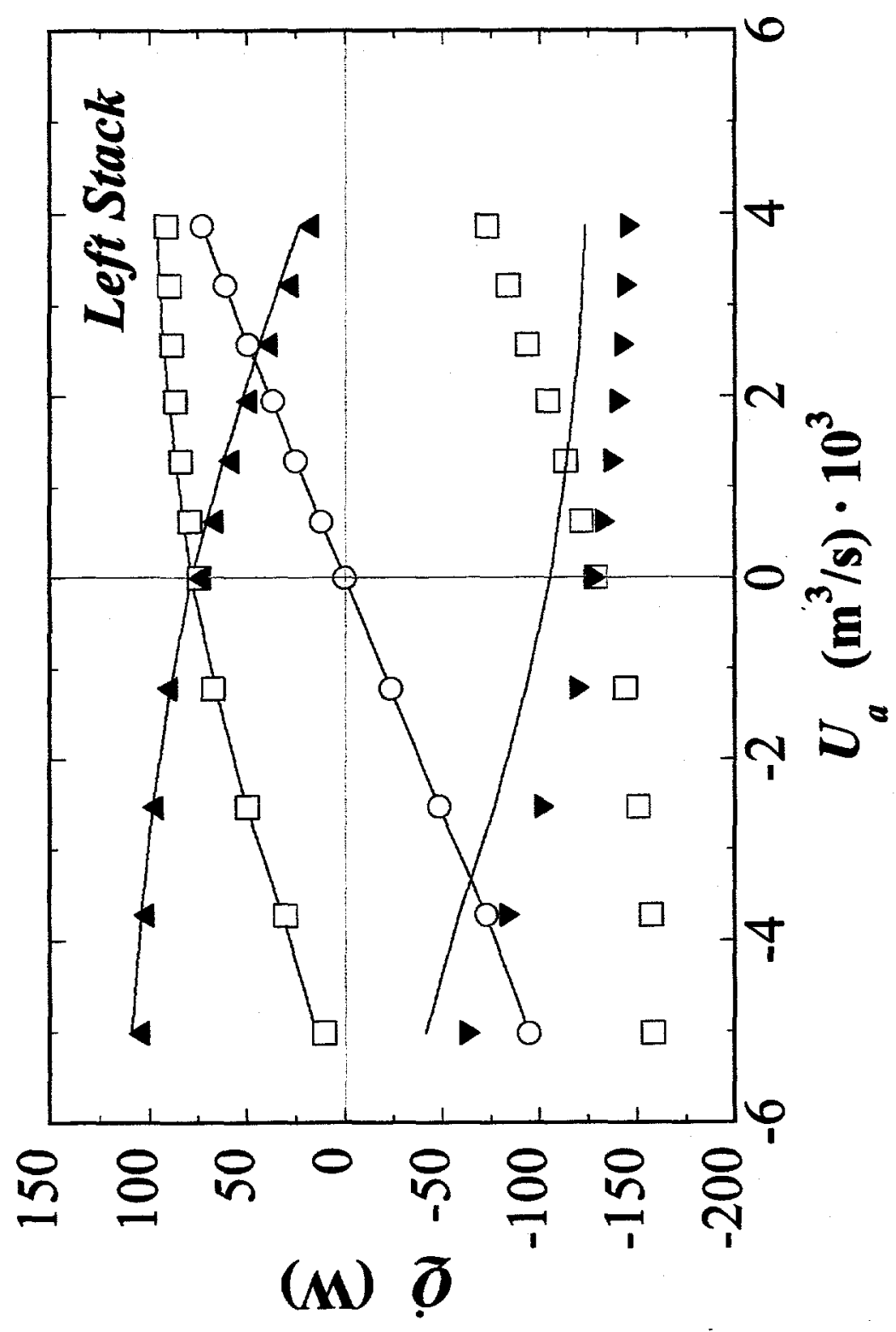

责

竘.

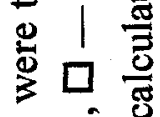

䒕

政

密和产

3

4

.을

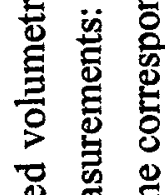

Ð

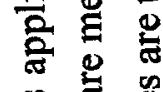

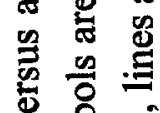

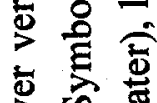

के छّ

용

离 +

事

몽

\%

要

它客

总量量 
total refrigerator power increase with $U_{a}$ clearly demonstrates the stacks' increased capacity to pump heat as the temperature at which the total heat load is applied shifts from $T_{C}$ to a continuum of temperatures $>T_{C}$. The increase in total refrigeration power as $U_{a}$ is changed from 0 to $5.21 \times 10^{-3} \mathrm{~m}^{3} / \mathrm{s}$ corresponds to a $22 \%$ improvement in First Law performance index.

The uncertainty intervals for the $\dot{Q}_{c}, \Delta \dot{H}_{0}$, and $U_{a}$ data sets typically lie within the areas covered by the symbols used to represent their values. The magnitude of the hotend-heat rejection rate also increased with applied flow rate. Accuracy limitations in the rotameter measurements caused the data set for the hot-end heat rejection rate to be of lower quality than the other measurements shown in Figure 22. These rotameter data are omitted from Figures 22 but are tabulated in Appendix B. Another indication of the hotend heat-rejection rate is given from the heat balance calculation represented by the dark inverted triangles. The consistent $24-\mathrm{W}$ offset between the acoustic-model calculation line and $\dot{Q}_{H}$ measured by the power balance is entirely attributable to the difference in computed acoustic power shown in Figure 19.

The general trends for the right-stack data set shown in Figure 22 agree qualitatively with the predictions from the acoustic model. The small quantitative discrepancy between the values for $\dot{Q}_{C}$ are believed due to the previously discussed hardware imperfections. The discrepancy in the measured temperature profiles along the left and right stacks were 
typically $0.5^{\circ} \mathrm{C}$ in mid stack and correspond to observed differences in measured $\dot{Q}_{C}$ of $10 \mathrm{~W}$ out of $100 \mathrm{~W}$ total cooling power per stack. The left-stack data shown in Figure 23 agrees closely with the acoustic model calculation. The data points representing $\dot{Q}_{H}$ measured with the rotameter were omitted from Figure 23 because of the clear inaccuracy of their readings. The close agreement between the data on the left stack and the linear acoustic model are suggestive of stack geometry closer to the rectangular-pore ideal than the data for the right stack. The complete data set collected for both stacks is included in Appendix B. Note the symmetry about the $\dot{Q}=0$ axis between the refrigerator and heater results. The value of $\dot{Q}_{H}$ for the open-cycle thermoacoustic heater mirrors the value of $\dot{Q}_{C}$ for the open-cycle thermoacoustic refrigerator. This symmetry results from holding constant $\left|p_{1}\right| / p_{0}, T_{H}$, and $T_{C}$ during these tests.

\section{Performance Indices for the Stack}

The performance indices introduced at the end of Chapter 2 will now be used to evaluate the performance of the open-cycle thermoacoustic refrigerator. To allow a presentation of the results in completely dimensionless form, the dimensionless applied volumetric flow rate, $\left\langle\xi_{a}\right\rangle$, introduced in Equation (13), will be used as the independent variable to describe applied flow rate. The correspondence between dimensionless applied volumetric flow rate and the dimensional volumetric flow rate used in the preceding plot can be found in the reduced data presented in Appendix B. 
Figure 24 is a plot of two First Law performance indices versus dimensionless applied volumetric flow rate for the left stack. The ordinate $\bar{\beta}_{2}$ on Figure 24 applies to the data represented by the open circles. These points show the performance index for the open-cycle thermoacoustic heater defined in Table 4. The Table 4 definition is modified to use the acoustic power delivered to the left side of the resonator in the denominator

$$
\bar{\beta}_{2}=\frac{\dot{Q}_{H}+\dot{m}\left(h_{C}-h_{H}\right)}{\dot{W}_{a c}}
$$

The numerator of Equation (79) corresponds to the open square symbols in Quadrant III of Figure 23. The numerator of the performance index $\beta_{1}$ on Figure 24 corresponds to the open square symbols in Quadrant I of Figure 23. The performance index $\beta_{1}$ on Figure 23 represents the Refrigerator Mode \#1 performance index defined in Table 4 and modified in a similar manner to Equation (79).

$$
\beta_{1}=-\frac{\left[\dot{Q}_{C}+\dot{m}\left(h_{H}-h_{C}\right)\right]}{\dot{W}_{a c}}
$$

A clear positive slope in Figure 24 is apparent in the open-cycle thermoacoustic Refrigerator Mode \#1 performance index with applied flow rate. As previously mentioned, there is a $22 \%$ improvement in Refrigerator Mode \#1 performance index as the dimensionless applied volumetric flow rate increases from 0.00 to 0.02 and like 


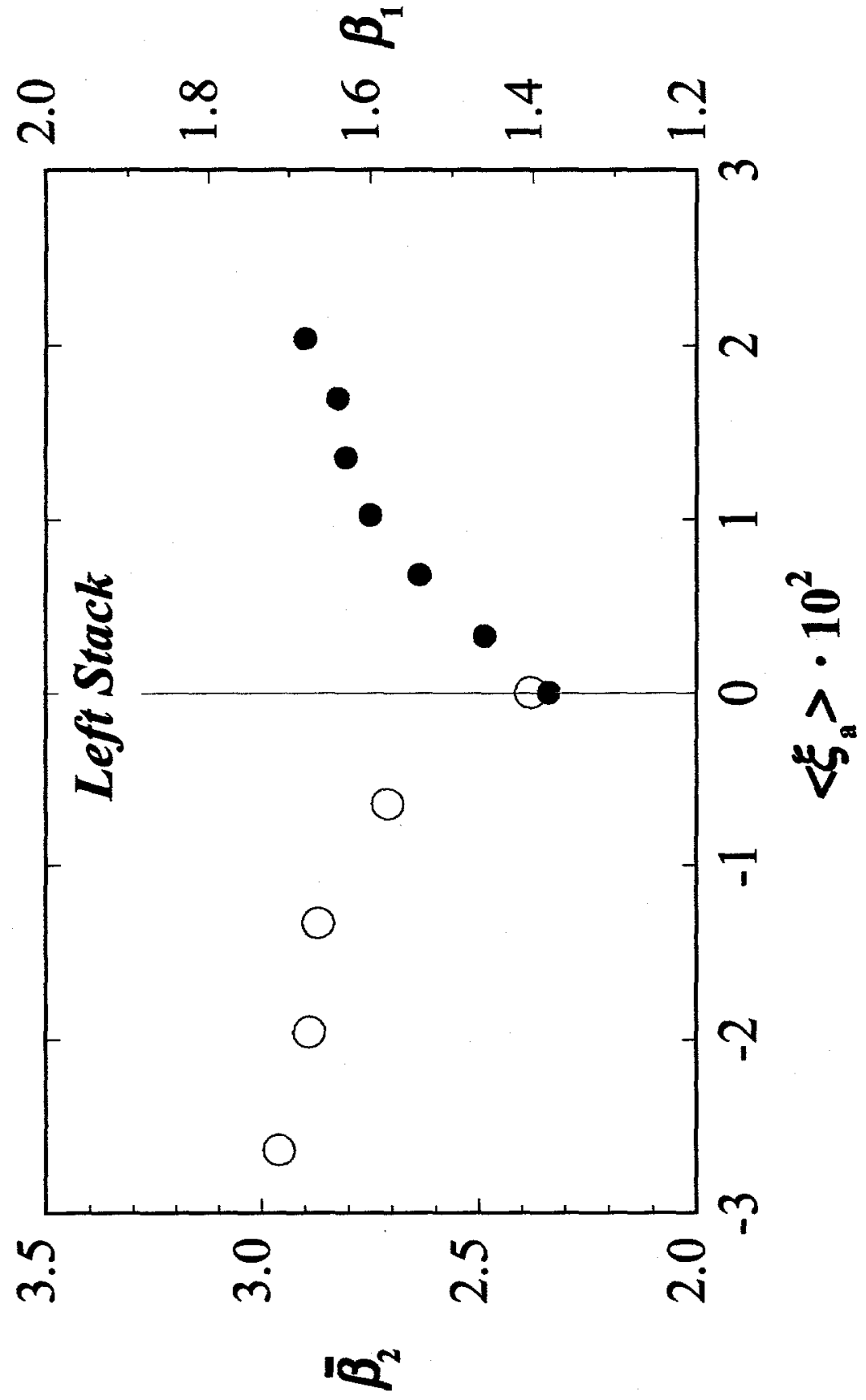

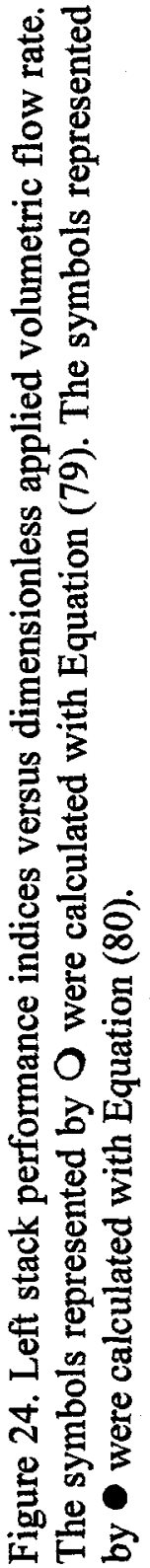


improvement in heater performance index as $\left\langle\xi_{a}\right\rangle$ changes from 0.00 to -0.03 . Similar trends are seen for the right stack plotted in Figure 25. The higher apparent performance index on the right stack is due to the previously discussed stack imperfections. Although the acoustic power supplied by each loudspeaker pair was balanced to within a few percent, additional power was absorbed by the right stack from a traveling-wave component, originating at the left speaker pair and superimposed on the standing wave. Additional power was absorbed on the right stack from viscous dissipation along the misaligned monofilaments. This added power accounts for the apparent side-to-side asymmetry in performance index.

The improvement obtained for the applied flow refrigerator can be normalized by taking a ratio between open-cycle and closed-cycle performance index

$$
\left(\frac{\beta_{1, o p e n}}{\beta_{1, \text { closed }}}\right)_{a c c u a l}=\left(\frac{\dot{W}_{a c}}{\dot{Q}_{C}}\right)_{\text {closed }}\left(\frac{\dot{Q}_{C}+\dot{m}\left(h_{H}-h_{C}\right)}{\dot{W}_{a c}}\right)_{\text {open }}
$$

The maximum theoretical improvement in performance index with applied flow rate can be found by considering the ideal refrigerator shown on the right side of Figure 26 . This refrigerator is configured so that a gaseous thermoacoustic working fluid at the reference temperature (taken in this case to be $T_{H}$ ) can be cooled to $T_{C}$ through either of two paths. Enthalpy from the gas stream flowing through the first path, equivalent to $\dot{Q}_{C}$, can be absorbed at $T_{C}$ in a single stage using power $\dot{W}_{\min }^{0}$. Alternatively, enthalpy can be 


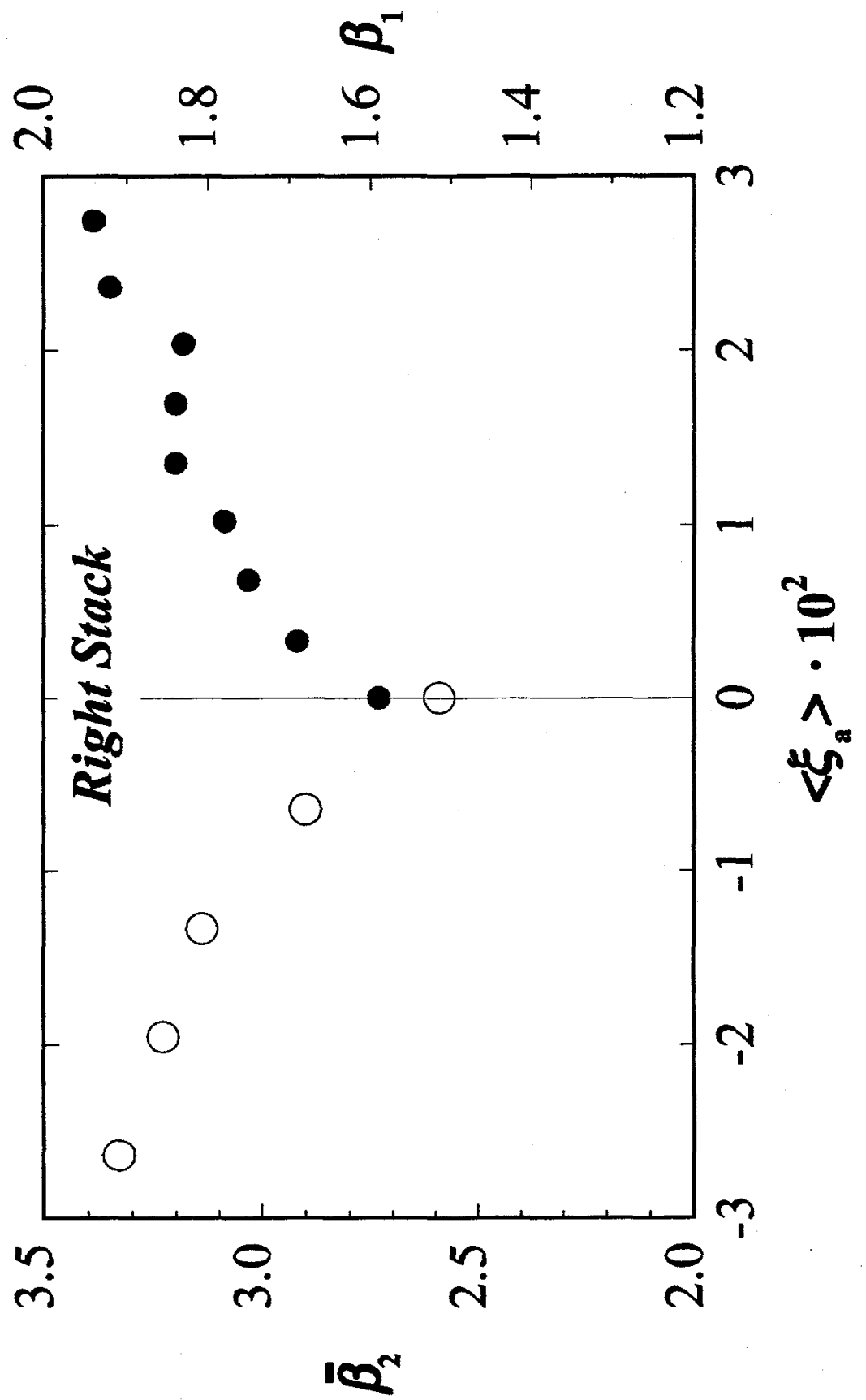

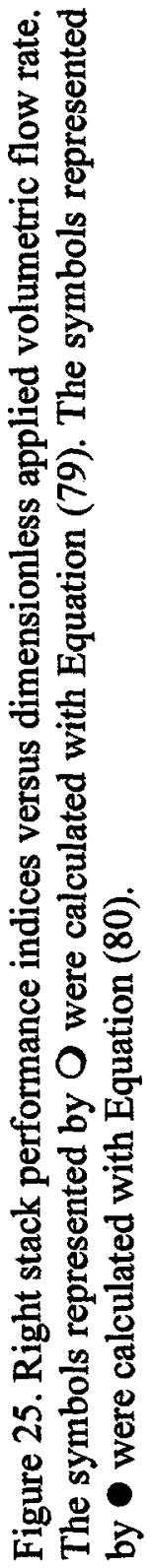


removed from the gas stream that flows through the second path at several intermediate temperatures shown on Figure 26. The maximum theoretical performance index improvement occurs if all of the gas is directed through the second path when that second path is comprised of an infinite number of intermediate stages. The ratio of the minimum power needed to complete the first process path to the power needed to complete the second process path is

$$
\left(\frac{\dot{W}_{\text {min,closed }}}{\dot{W}_{\text {min,open }}}\right)_{\text {ideal }}=\frac{\left(T_{H}-T_{C}\right)^{2}}{T_{C}\left(T_{H}-T_{C}-T_{H} \ln \left(T_{H} / T_{C}\right)\right)}
$$

For a refrigerator operating near room temperature with $T_{H}-T_{C} \ll<T_{H}$, the value of this ratio is about two. If the applied flow is divided between the two paths, the open-cyclerefrigerator-ideal-performance index ratio becomes

$$
\left(\frac{\beta_{1, \text { open }}}{\beta_{1, \text { closed }}}\right)_{\text {ideal }}=\frac{\dot{Q}_{C}}{\dot{Q}_{C}+\Delta \dot{H}_{0}}-\frac{\Delta \dot{H}_{0}}{\dot{Q}_{C}+\Delta \dot{H}_{0}} \frac{\left(T_{H}-T_{C}\right)^{2}}{T_{C}\left(T_{H}-T_{C}-T_{H} \ln \left(T_{H} / T_{C}\right)\right)}
$$

where $\Delta \dot{H}_{0}=\dot{m} c_{p}\left(T_{H}-T_{C}\right)$ was defined in Chapter 2. In a similar manner, ratios can be defined for the performance index used for the open-cycle heater shown on Figure 26. The measured ratio of performance indices for the Heater Mode \#3 configuration is 

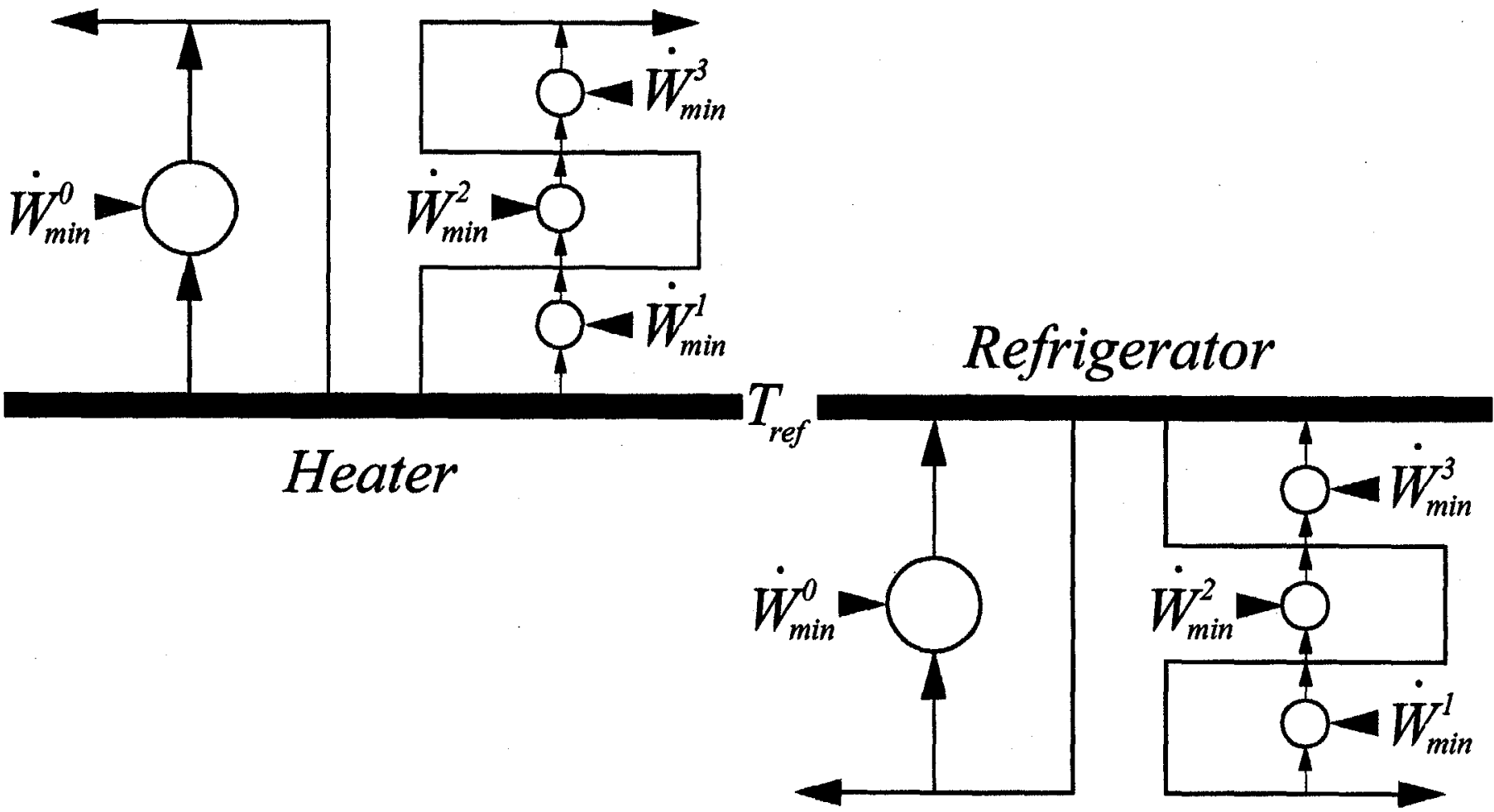

Figure 26. Comparison of single stage to multi-stage modes of operation for the devices previously defined in Figure 5: "Device B" open cycle heater and the "Device A" open cycle refrigerator. 


$$
\left(\frac{\bar{\beta}_{2, o p e n}}{\bar{\beta}_{2, \text { closed }}}\right)_{a c t u a l}=\left(\frac{\dot{W}_{a c}}{\dot{Q}_{H}}\right)_{\text {closed }}\left(\frac{\dot{Q}_{H}+\dot{m}\left(h_{C}-h_{H}\right)}{\dot{W}_{a c}}\right)_{o p e n}
$$

Using $T_{C}$ as the reference temperature for the ideal case yields

$$
\left(\frac{\bar{\beta}_{2, \text { open }}}{\bar{\beta}_{2, \text { closed }}}\right)_{\text {ideal }}=\frac{\dot{Q}_{H}}{\dot{Q}_{H}-\Delta \dot{H}_{0}}+\frac{-\Delta \dot{H}_{0}}{\dot{Q}_{H}-\Delta \dot{H}_{0}} \frac{\left(T_{H}-T_{C}\right)^{2}}{T_{H}\left(T_{C}-T_{H}-T_{C} \ln \left(T_{C} / T_{H}\right)\right)}
$$

These results are plotted in Figure 27 for the left stack and Figure 28 for the right stack. The dark symbols represent measured values and the open symbols represent ideal values. The ideal-performance-index ratio values for the left and right stacks differ. The difference reflects the dependence of the ideal value on the measured relation among $\Delta \dot{H}_{0}, \dot{Q}_{H}$, and $\dot{Q}_{C}$. The ideal values exceed the measured values for all of the data points on the refrigerator side. Oddly, several measured heater values slightly exceed their respective ideal values. This suggests error in the values $\dot{W}_{a c}, \dot{Q}_{c}$, and $U_{a}$ associated with small differences between two large values, $T_{H}-T_{C}$ and $T_{C} \ln \left(T_{C} / T_{H}\right)$, in the denominator of Equation (85). The minimum power to complete a both processes diminishes as more of the process fluid is diverted through an open-cycle path. 


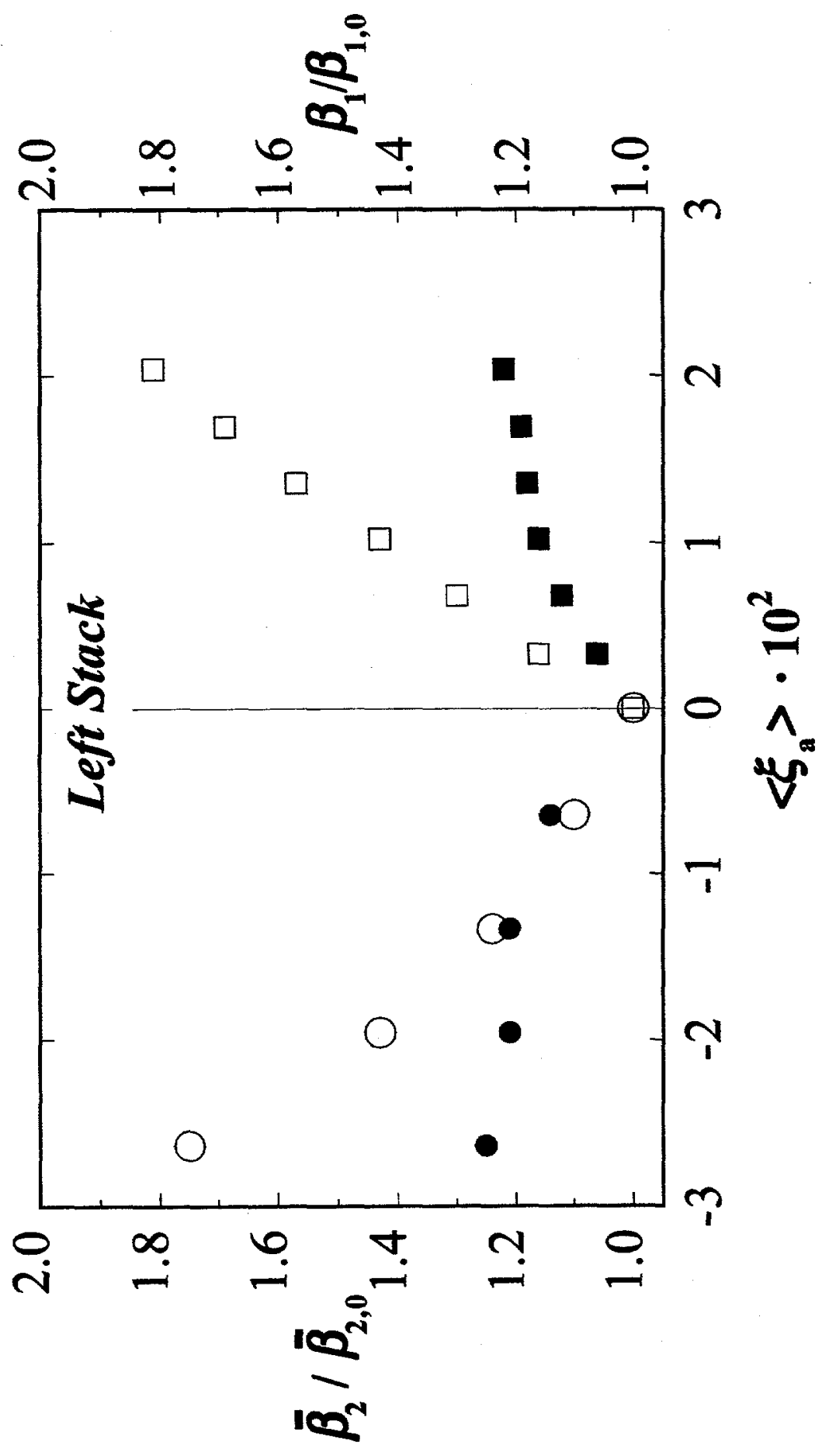

氶焉焉

$>\stackrel{\Xi}{ \pm}$

过范

E

巳 홍

胥

홍뭉

융

을 0

डั

융

응

它

$\circ$ F

$x$ 릉

要

$\Xi \Phi$

过

흐을

눈 $\frac{0}{0}$

昰

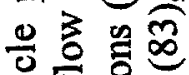

강

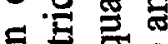

용 留

잉

웧응

4. 可要

은 욤 도

焉焉方

둘 के

ㄱ.气

을 옹을

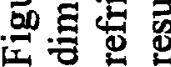




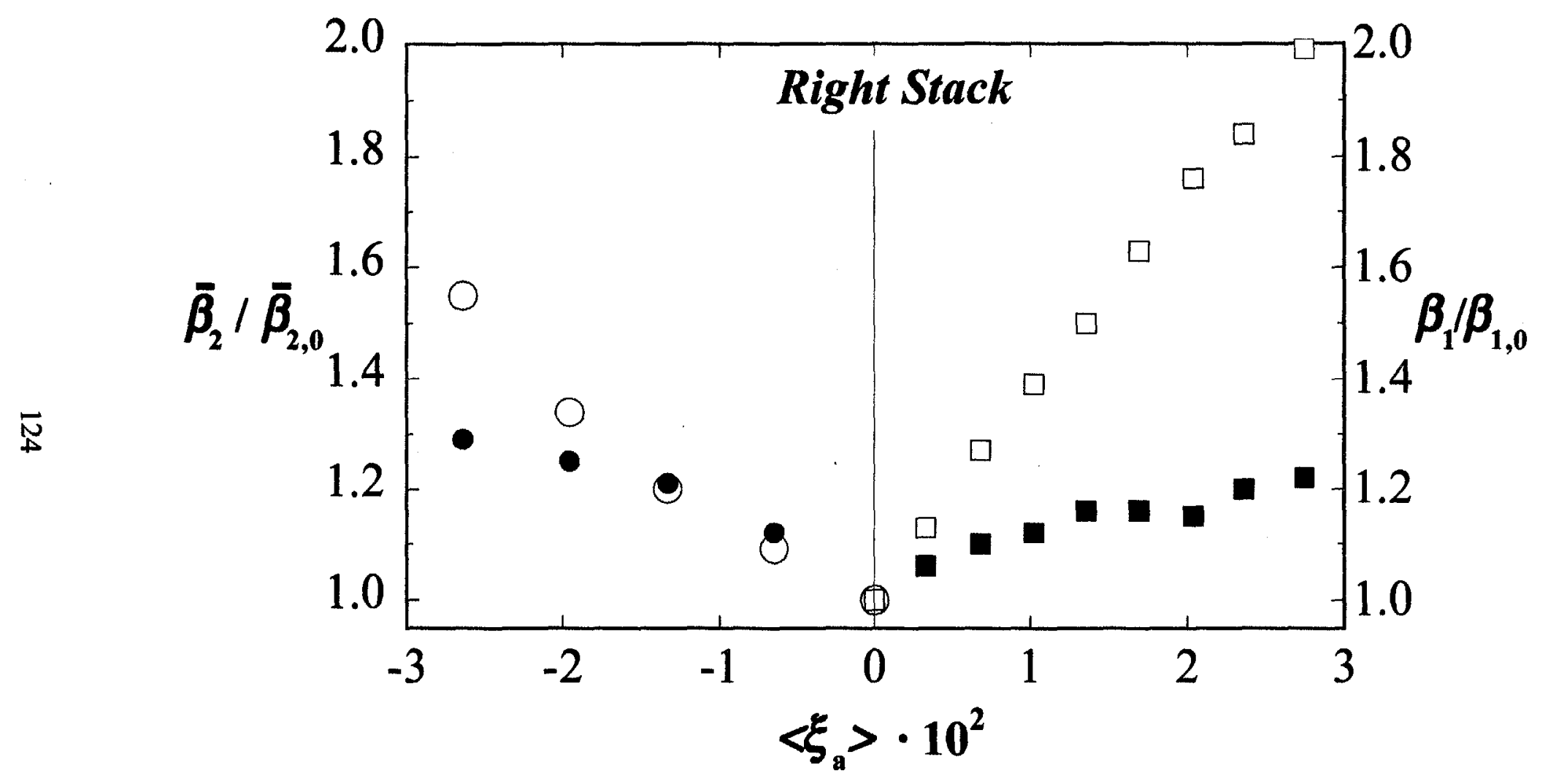

Figure 28. Same results as shown in Figure 27, but for the right stack. 
The Second Law effectiveness for a refrigerator is a ratio of the minimum to the actual power required to complete a defined process. The Table 4 definition for refrigerator Second Law effectiveness is changed to account for the acoustic power absorbed by the stack and the resonator

$$
\varepsilon_{1}=\frac{\dot{Q}_{c}\left(1-T_{H} / T_{C}\right)-\dot{m}\left(h_{C}-h_{H}\right)+\dot{m} T_{H}\left(s_{C}-s_{H}\right)}{\dot{W}_{a c}}
$$

The dark circles on Figure 29 (which map to the $\varepsilon_{1}$ ordinate) are plotted versus dimensionless applied volumetric flow rate for the left stack. Figure $\mathbf{3 0}$ shows a similar plot for the right stack. A consistent downward trend is apparent in Second Law effectiveness as the applied flow rate increases for both the right-stack and the left-stack data sets. The Second Law effectiveness for the stacks operating as open-cycle thermoacoustic heaters is also modified to account for the total acoustic power absorbed by the system:

$$
\bar{\varepsilon}_{2}=\frac{\dot{Q}_{H}\left(1-T_{C} / T_{H}\right)-\dot{m}\left(h_{C}-h_{H}\right)+\dot{m} T_{C}\left(s_{C}-s_{H}\right)}{\dot{W}_{a c}}
$$

The $\bar{\varepsilon}_{2}$ ordinates on Figures 29 and 30 correspond to the dark squares plotted on those figures. As for the open cycle refrigerator, the open cycle heater's Second Law effectiveness diminishes as the applied flow rate is increased. 

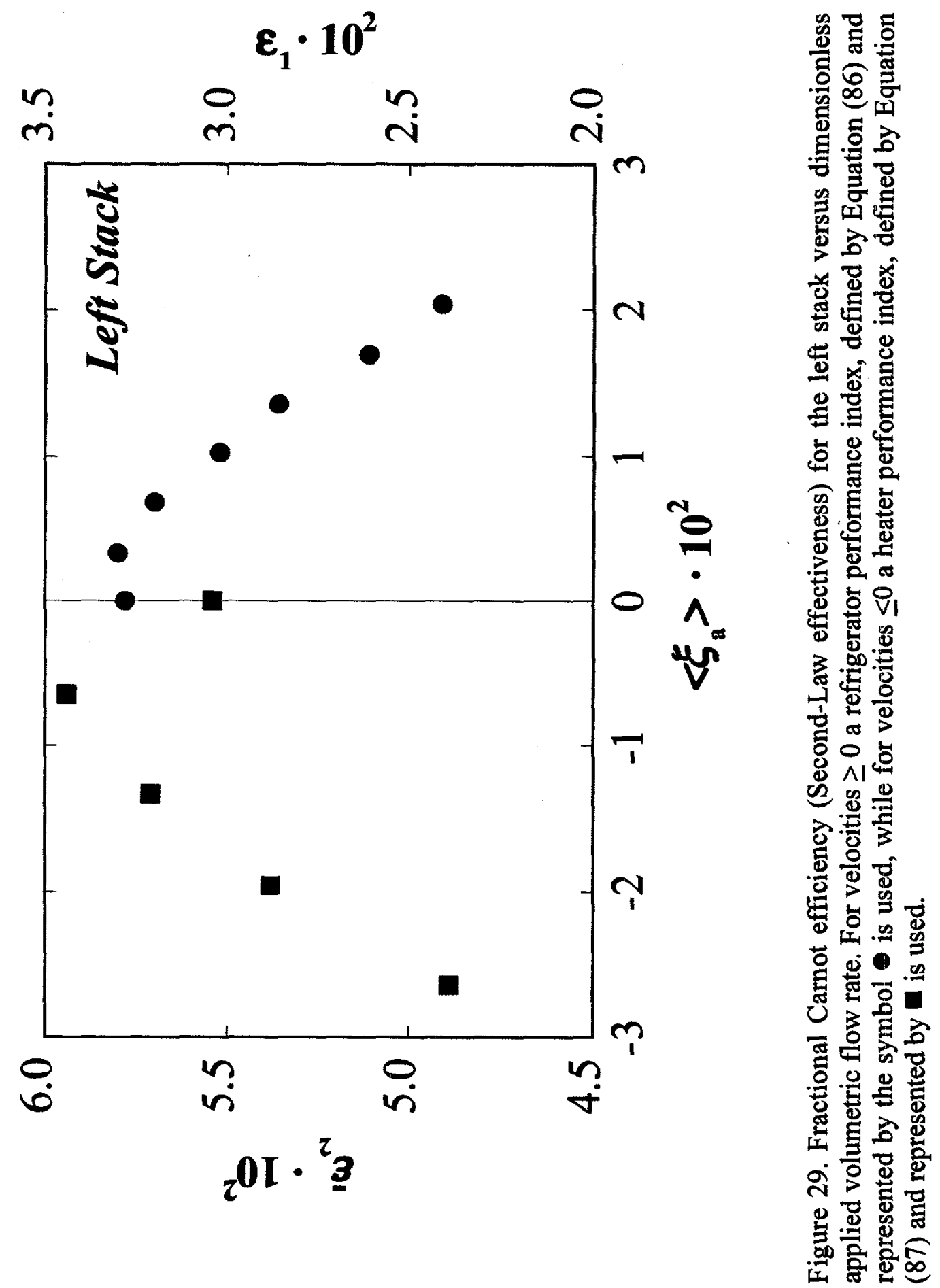


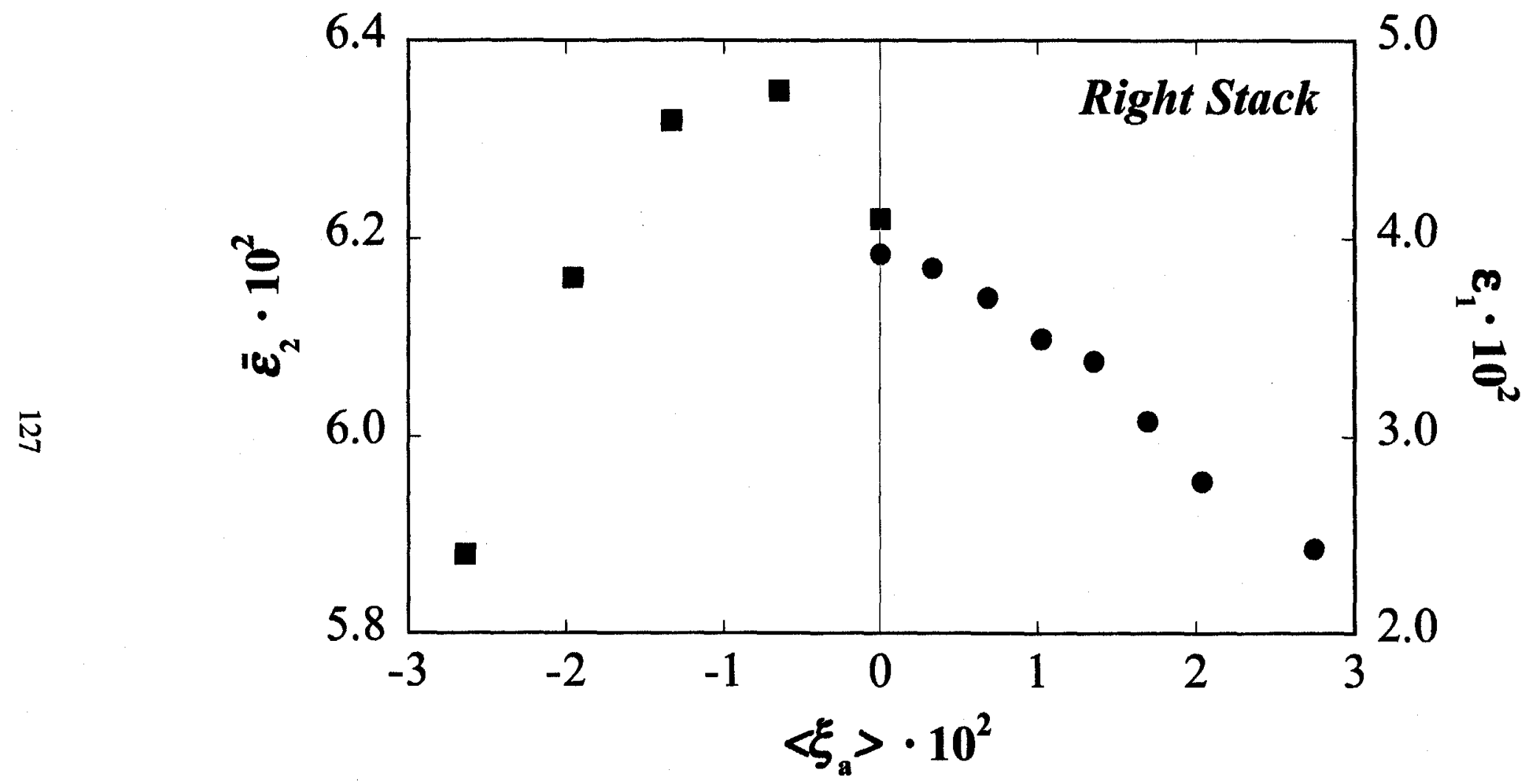

Figure 30. Fractional Carnot efficiency (Second-Law effectiveness) for the right stack versus dimensionless applied volumetric flow rate. For velocities $\geq 0$ a refrigerator performance index, defined by Equation (86) and represented by the symbol $\bullet$ is used, while for velocities $\leq 0$ a heater performance index, defined by Equation (87) and represented by is used. 
This result produces an apparent paradox: Second Law effectiveness diminishes with applied flow rate while First Law efficiency increases with applied flow rate. The paradox can be avoided if processes are viewed as distinct for each combination of applied flow rate and cold-end heat absorption. The effect of applied flow rate on Equation (86) diminishes the reversible power required to complete the process. That is, an ideal open-cycle refrigerator requires less reversible power to cool a gas stream from $T_{\text {ref }}$ to $T_{C}$ than a similar ideal single-stage refrigerator. This necessarily leads to a reduction in Second Law effectiveness for non-zero applied flow in the definitions given by Equations (86) and (87). This reduction in Second Law effectiveness with increasing applied flow rate is not limited to thermoacoustic devices. Second Law effectiveness, as defined in this thesis, provides an inadequate indication of the improvement in First Law efficiency realized in the transition from closed to open cycle operation. A quantitative comparison of Second Law effectiveness for two refrigerators operating at different applied-flow-rate-cold-end-heat absorption proportions is not strictly valid. However, the Second Law effectiveness defined by Equations (86) and (87) can be used to compare different devices, thermoacoustic or otherwise, operating with similar proportions of open and closed cycle heat absorption. 


\section{CHAPTER 5}

\section{CONCLUSIONS AND SUGGESTIONS FOR FUTURE WORK}

The open-cycle thermoacoustic device demonstrated here uses a gaseous-phase working fluid. Measured performance coefficients for this device were within the range normally associated with gas-refrigeration cycles. The superposition of applied flow on the thermoacoustic cycle was shown to enhance the First Law performance index for the device. However, the shape of the open thermoacoustic cycle in $T-s$ space indicates its inherent irreversibility, as proposed. It seems improbable that open thermoacoustic cycles, as described in Chapter 2, will ever approach the efficiency of phase-change cycle technologies. That being said, many factors other than efficiency can influence the selection of technology for real-world applications. Among these are construction and operating costs, efficiency, size, weight, and reliability. Practical (economic) arguments often favor solutions with non-optimal efficiency. For example, the reverse Brayton-cycle cooler used aboard turbine aircraft provides weight savings that indirectly leads to lower operating cost, despite poor First Law efficiency. Similar rationale for the use of the open 
thermoacoustic cycle may eventually be found. Several proposed applications of this technology are mentioned in a US patent application (Swift, Reid, and Ward, 1999) and have received attention elsewhere (Landsberg, 1998).

Even if practical open-cycle thermoacoustic devices are never developed, there are several ideas presented in the thesis that could be used to improve other thermodynamic cycles. The combination of the thermodynamic and process working fluids is an old but worthy idea that allowed the mass production of the affordable automobiles early in this century. Perhaps this combination could be more generally applied. Cycle staging by open flow superposition is a novel aspect of this work and accounts for the measured improvements in First Law efficiency. Most open-cycle devices take their process fluid through a single series of cyclic steps. This work demonstrated the possible advantages of taking the process fluid though multiple stages.

The Chapter 2 discussion was a modest development of the rich potential contained in the equation set given in Appendix A. The inviscid-Lagrangian pathline calculation could be extended to consider viscosity starting with the Appendix A relations. Also, the connection between the various enthalpy flux ratios (generically symbolized with the Greek letter $\Xi$ ) and the Chapter 2 performance indices was not made explicit.

Several novel views emerge from the Chapter 2 discussion and the Chapter 4 results. Applied flow superposition blurs the distinction between a cycle and a process. The open thermoacoustic cycle can be viewed on at least two different length scales: the individual cycle loop of order $\left|x_{1}\right|$ and the overall process across the stack of order $L$. The 
method chosen to control these tests produced performance symmetries between the open-cycle thermoacoustic refrigerator and the open-cycle thermoacoustic heater. Such symmetry is revealed by casual inspection of Figure 22 and Figure 23. Another symmetry can be produced by a somewhat artificial coordinate system manipulation. Equation (28) can be expanded to include simultaneously the temperature profiles in the presence of applied flow for the heater, engine, engine-heater, and refrigerator devices in a four-quadrant plot. A general definition of this expanded inviscid temperature profile is

$$
\Theta(\bar{x})=\frac{T(\bar{x})-T_{\text {ref }}}{T_{c r i t}-T_{\text {ref }}}=\Gamma \cdot \frac{1-e^{(-1)^{(m+o)} \Xi \operatorname{sign}(\bar{x}) \bar{x}}}{1-e^{(-1)^{(m+o)} \Xi}} \cdot(-1)^{(m+n)} \cdot \operatorname{sign}(\bar{x})
$$

where $\Xi$ is defined to be positive at all $\bar{x}$. The indices $m, n$, and $o$ determine the quadrant in which a given device resides. Figure 31 shows sample results from this equation for the case $\Gamma=1, \Xi=5, m=0$ (the dashed curve), $m=1$ (the solid curve), $n=1, o=0$. Note that the axis $\Theta=0$ can be viewed to represent the reference condition for the heater, engine, and refrigerator devices. The meaning of the $\Theta=0$ as a reference condition for the engine-heater is less clear. Perhaps some significance can be found in these symmetries.

The other open-cycle thermoacoustic devices, the engine and the engine-heater, first introduced in Chapter 2, were not examined in any detail. They offer fertile ground for experimentation and development. The efficiency limitations in thermoacoustic- 
related (and other) gas cycles may not be inherent. A recently developed traveling-wave engine based on a Stirling cycle has demonstrated Second Law effectiveness of $40 \%$ (Backhaus and Swift, 1999). Incorporating open-cycle acoustics into higher efficiency cycles, with some form of phase change or in-cycle combustion, might eventually produce useful refrigeration or propulsion configurations. One tantalizing prospect: an open-cycle acoustic-ram jet hybrid operating on an open-acoustic pulse-combustion cycle at low speed, transitioning to ram jet operation at high speed.

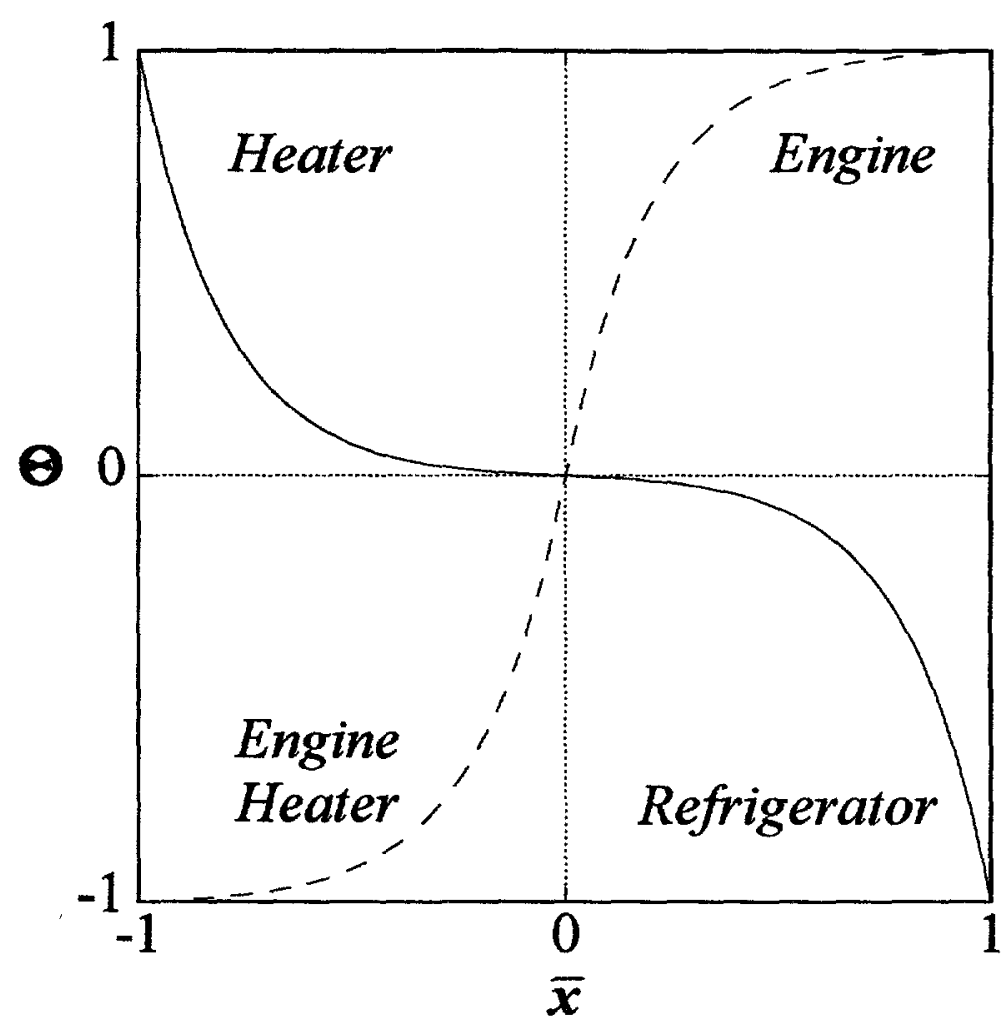

Figure 31. A symmetry relation found in the exponential temperature profiles among the four types of open-cycle thermoacoustic device defined in Chapter 2. 


\section{APPENDIX A}

\section{PROPOSED EQUATIONS FOR APPLIED FLOW THROUGH STACK}

The following equation set was derived by Greg Swift based on the linear acoustic approximation first developed by Nicolas Rott. A summary of Rott's equations as applied to conventional thermoacoustic engines and refrigerators is given in (Swift, 1988). This equation set extends that application to stacks with non-zero flow.

Conventional thermoacoustics is based on Rott's wave equation and energy-flux equation. These equations are derived in the acoustic approximation from the momentum, continuity, and energy equations for fluids. As with simpler onedimensional acoustic wave equations, Rott's wave equation may be rewritten as two coupled first-order differential equations in the oscillatory pressure and oscillatory volumetric velocity. Rott's energy-flux equation may be regarded as a first order differential equation in mean temperature coupled to the pressure and velocity equations. 
To derive corresponding equations in the presence of applied flow, the momentum, continuity, and energy equations are used. The dependent variables used in these equations and their respective domains are

$$
\begin{gathered}
p=p_{0}(x)+\operatorname{Re}\left[p_{1}(x) e^{i \omega x}\right] \\
u=u_{a}(x, y, z)+\operatorname{Re}\left[u_{1}(x, y, z) e^{i \omega x}\right] \\
v=\operatorname{Re}\left[v_{1}(x, y, z) e^{i \alpha x}\right] \\
w=\operatorname{Re}\left[w_{1}(x, y, z) e^{i \omega x}\right] \\
T=T_{0}(x)+T_{0}^{\prime}(x, y, z)+\operatorname{Re}\left[T_{1}(x, y, z) e^{i \omega x}\right] \\
\rho=\rho_{0}(x)+\rho_{0}^{\prime}(x, y, z)+\operatorname{Re}\left[\rho_{1}(x, y, z) e^{i \omega x}\right] \\
\mu=\mu_{0}(x)+\mu_{0}^{\prime}(x, y, z)+\operatorname{Re}\left[\mu_{1}(x, y, z) e^{i \omega x}\right] \\
K=K_{0}(x)+K_{0}^{\prime}(x, y, z)+\operatorname{Re}\left[K_{1}(x, y, z) e^{i \alpha x}\right]
\end{gathered}
$$

Relations similar to (A 8) apply to thermodynamic properties such as sound speed, entropy, internal energy, and enthalpy. Of most interest are $T_{0}, p_{1}$, and the volumetric velocities $U_{1}, U_{a}$ :

$$
U_{1}(x)=\int_{A_{\text {fadid }}} u_{1}(x, y, z) d y d z
$$




$$
U_{a}(x)=\int_{A_{\text {fuid }}} u_{a}(x, y, z) d y d z
$$

where the integrals are taken over the cross sectional area $A_{\text {fuid }}$ open to the fluid. The ratios $p_{1} / p_{0}$ and $v_{1} / a$ are assumed to be small. All terms that are first order in smallness are kept in the momentum and continuity equations, and terms second order and larger are kept in the energy equation.

Viscosity, thermal conductivity, sound speed, and other thermophysical properties depend on pressure and temperature. These variables are small and are treated as timeindependent functions, which are only dependent on zeroth order temperature. For clarity, the subscript " 0 " will be omitted on such variables, reserving its use for variables such as density and temperature which have both applied and oscillatory components.

Neglecting the second (bulk) viscosity, the momentum equation for a general Newtonian fluid is

$$
\rho[\partial \mathbf{v} / \partial t+(\mathbf{v} \cdot \nabla) \mathbf{v}]=-\nabla p+\frac{\partial}{\partial \mathrm{x}_{k}}\left[\mu\left(\frac{\partial v_{i}}{\partial \mathrm{x}_{k}}+\frac{\partial v_{k}}{\partial \mathrm{x}_{i}}-\frac{2}{3} \delta_{i k} \frac{\partial v_{l}}{\partial \mathrm{x}_{l}}\right)\right]
$$

The $x$-component of (A 11) is the most significant and is kept. Equations (A1) - (A7) are substituted for all variables, and all terms larger than second order are preserved. The $x$ derivatives in the viscous term are small compared to the $y$ - or $z$-derivatives, by 
roughly the ratio of viscous penetration depth to wavelength. The time-independent terms and the terms proportional to $e^{i \omega x}$ must equate separately, so the equation splits cleanly in two

$$
\begin{gathered}
i \omega \rho_{0} u_{1}=-d p_{1} / d x+\mu \nabla_{y, z}^{2} u_{1} \\
0=-d p_{0} / d x+\mu \nabla_{y, z}^{2} u_{a}
\end{gathered}
$$

The oscillatory equation, Equation (A12), is unchanged by the presence of applied flow. It is a differential equation for $u_{1}(y, z)$, with the boundary condition that $u_{1}=0$ on solid boundaries. Its solution is

$$
u_{1}=\frac{i}{\omega \rho_{0}}\left[1-h_{v}(y, z)\right] \frac{d p_{1}}{d x}
$$

where $h_{v}(y, z)$ depends on the specific geometry under consideration (Arnott, 1991). Integrating with respect to $y$ and $z$, the oscillatory volumetric velocity is obtained

$$
U_{1}=\frac{i A_{\text {fluid }}}{\omega \rho_{0}}\left(1-f_{v}\right) \frac{d p_{1}}{d x}
$$

where for fluid flow between parallel plates 


$$
f_{v}=\frac{\tanh \left[(1+i) r_{h} / \delta_{v}\right]}{(1+i) r_{h} / \delta_{v}}
$$

and for rectangular pores

$$
f_{v}=\frac{64}{\pi^{4}} \sum_{m n \text { odd }} \frac{1}{m^{2} n^{2} Y_{m n}(\lambda)}
$$

where

$$
Y_{m n}(\lambda)=1+\left(i \pi^{2} / \lambda^{2}\right)\left[\left(b^{2} m^{2}+a^{2} n^{2}\right) /(a+b)^{2}\right] \quad \text { and } \quad \lambda=\sqrt{2} r_{h} / \delta_{v}
$$

The applied flow equation, Equation (A13), expresses the fact that viscosity and applied flow cause a pressure gradient. The applied flow pressure gradient $d p_{0} / d x$ is of second order so it can be neglected and $p_{0}$ can be regarded as constant.

In the continuity and energy equations, expressions for oscillatory temperature $T_{1}$ and its spatial average over $y$ and $z$, denoted $\left\langle T_{1}\right\rangle$, are needed. Starting with a general form of the equation of heat transfer found in Landau and Lifshitz (1987)

$$
\rho T\left(\frac{\partial s}{\partial t}+\mathbf{v} \cdot \nabla \mathrm{s}\right)=\nabla \cdot(K \nabla T)+\frac{1}{2} \mu\left(\frac{\partial v_{i}}{\partial \mathrm{x}_{k}}+\frac{\partial v_{k}}{\partial \mathrm{x}_{i}}-\frac{2}{3} \delta_{i k} \frac{\partial v_{l}}{\partial \mathrm{x}_{l}}\right)^{2}+\zeta(\nabla \cdot \mathbf{v})^{2}
$$


using $d s=\left(c_{p} / T\right) d T-(\beta / \rho) d p$, keeping terms to first order only, and separating timeindependent terms from terms proportional to $e^{i \omega x}$ yields

$$
\begin{gathered}
\rho_{0} c_{p}\left(i \omega T_{1}+u_{1} d T_{0} / d x\right)-i \omega T_{0} \beta p_{1}=K \nabla_{y, z}^{2} T_{1} \\
\rho_{0} c_{p} u_{a} d T_{0} / d x=K \nabla_{y, z}^{2} T_{0}^{\prime}
\end{gathered}
$$

Using Equation (A14) for $u_{1}$, and with boundary condition $T_{1}=0$ on solid boundaries, Equation (A19) is a first-order ordinary differential equation for $T_{1}(x, y)$, similar in form to Equation (A12) for $u_{1}(x, y)$. Exploiting the similarity between Equations (A12) and (A19), the solution for $T_{1}$ can be written

$$
T_{1}=\frac{T \beta}{\rho c_{p}}\left[1-h_{v}(y, z)\right] p_{1}+\frac{i}{A_{\text {fluid }} \omega\left(1-f_{v}\right)} \frac{d T_{0}}{d x}\left[1-\frac{h_{k}(y, z)}{1-\sigma}+\frac{\sigma h_{v}(y, z)}{1-\sigma}\right] U_{1}
$$

and the spatial average can be written

$$
\left\langle T_{1}\right\rangle=\frac{T \beta}{\rho c_{p}}\left(1-f_{\mathrm{\kappa}}\right) p_{1}+\frac{i}{A_{f u u i d} \omega\left(1-f_{v}\right)} \frac{d T_{0}}{d x}\left(1-\frac{f_{x}-\sigma f_{v}}{1-\sigma}\right) U_{1}
$$


where $f_{\kappa}$ and $h_{x}$ are the same as $f_{v}$ and $h_{v}$, but with $\delta_{v}$ replaced by the thermal penetration depth $\delta_{\kappa}=\sqrt{2 K / \omega \rho_{0} c_{p}}$. Note that Equation (A20) and therefore $T_{0}^{\prime}$ is second order so related changes in thermophysical properties such as $\rho_{0}^{\prime}, \mu_{0}^{\prime}$, and $K_{0}^{\prime}$ are of negligible order and can be ignored.

A form of the continuity equation found in Landau and Lifshitz (1987) is

$$
\partial \rho / \partial t+\nabla \cdot(\rho \mathbf{v})=0
$$

This equation can be integrated with respect to $y$ and $z$, to obtain, to first order

$$
\begin{gathered}
i \omega A_{\text {fluid }}\left\langle\rho_{1}\right\rangle+\frac{d\left(\rho_{0} U_{1}\right)}{d x}=0 \\
\frac{d\left(\rho_{0} U_{a}\right)}{d x}=0
\end{gathered}
$$

Using $d \rho=-\rho \beta d T+\left(\gamma / a^{2}\right) d p$, the densities can be expressed to lowest order as

$$
\begin{gathered}
\left\langle\rho_{1}\right\rangle=-\rho_{0} \beta\left\langle T_{1}\right\rangle+\left(\gamma / a^{2}\right) p_{1} \\
d \rho_{0} / d x=-\rho_{0} \beta d T_{0} / d x
\end{gathered}
$$

Substituting these into Equations (A24) and (A25), using Equation (A22) for $\left\langle T_{1}\right\rangle$, yields 


$$
\begin{gathered}
\frac{i \omega A_{\text {fluid }}}{\rho_{0} a^{2}}\left[1+(\gamma-1) f_{\kappa}\right] p_{1}-\frac{\beta\left(f_{\kappa}-f_{v}\right)}{\left(1-f_{v}\right)(1-\sigma)} \frac{d T_{0}}{d x} U_{1}+\frac{d U_{1}}{d x}=0 \\
\frac{d U_{a}}{d x}-\beta \frac{d T_{0}}{d x} U_{a}=0
\end{gathered}
$$

In the summary below, Equation (A28) will be solved for $d U_{1} / d x$ and Equation (A29) for $d U_{a} / d x$, regarding these approximations to the continuity equation as the sources of $x$ dependence of $U_{1}$ and $U_{a}$.

Another form of the energy equation found in Landau and Lifshitz (1987) is used to find an expression for $d T_{0} / d x$, using the fact that the total time-averaged energy flux along the stack must be independent of $x$, because the side walls of the stack are rigid and thermally insulated and the system is in time-averaged steady state. The general law of conservation of energy is given by the equation

$$
\frac{\partial}{\partial t}\left(\frac{1}{2} \rho v^{2}+\rho \varepsilon\right)=-\nabla \cdot\left[\rho v\left(\frac{1}{2} v^{2}+h\right)-v \cdot \Sigma-K \nabla T\right]
$$

where $\varepsilon$ and $h$ are internal energy and enthalpy per unit mass, respectively, and $\Sigma$ is the viscous stress tensor. The term proportional to $\mathrm{v} v^{2}$ is of third order and can be ignored. Time averaging and integrating the remaining terms over the cross-sectional area of the stack yields 


$$
0=\frac{d}{d x}\left[A_{\text {fluid }}\langle\overline{\rho u h}\rangle-A_{\text {fluid }}\langle\overline{K \partial T / \partial x}\rangle-A_{\text {solid }}\left\langle K_{\text {solid }} \partial T_{\text {solid }} / \partial x\right\rangle_{\text {solid }}-A_{\text {fluid }}\left\langle\overline{(\bar{v} \cdot \Sigma)_{x}}\right\rangle\right]
$$

with the overbar denoting time average, \langle\rangle denoting the spatial average over the fluid cross section, and \langle\rangle$_{\text {solid }}$ denoting the spatial average over the solid cross section. The quantity within the square brackets is the time-averaged energy flux $\dot{H}$ along $x$. The second and third terms of Equation (A31) contain terms of all orders, but only the zeroorder terms are significant. The viscous tensor part of Equation (A 31) also has many terms, with the largest of order $\mu u_{1}^{2} / \lambda$, but $\overline{\rho u h}$ is of order $p_{1} u_{1} \simeq \rho_{0} a u_{1}^{2}$, and

$$
\frac{\mu u_{1}^{2}}{\lambda} \frac{1}{\rho_{0} a^{2} u_{1}}=\frac{v}{\lambda a}<1
$$

so the viscous term is negligible compared to the first term in Equation (A 31). Expanding the first term in Equation (A 31) to second order gives

$$
A_{\text {fluid }}\left\langle\rho_{0} u_{a} h_{0}+\overline{\rho_{1} u_{1}} h_{0}+\rho_{0} \overline{u_{2}} h_{0}+\rho_{0} \overline{u_{1} h_{1}}\right\rangle
$$

where there are already five terms set equal to zero because the time average of first order quantities is zero. The applied mass flow is assumed to be fixed at $\rho_{0} U_{a}=A_{\text {fluid }}\left\langle\rho_{0} u_{a}\right\rangle$, so 
that the second-order time averaged mass flux $A_{\text {fluid }}\left\langle\overline{\rho_{1} u_{1}}+\rho_{0} \overline{u_{2}}\right\rangle=0$ and Equation (A 33) reduces to

$$
A_{\text {fluid }}\left\langle\rho_{0} u_{a} h_{0}+\rho_{0} \overline{u_{1} h_{1}}\right\rangle
$$

Using $d h=c_{p} d T+[(1-T \beta) / \rho] d p$, and combining this with the other significant terms from Equation (A31), yields

$$
\dot{H}_{2}=\rho_{0} U_{a} h_{0}+A_{\text {fluid }} \rho_{0} c_{p}\left\langle\overline{T_{1} u_{1}}\right\rangle+\left(1-T_{0} \beta\right) \overline{p_{1} U_{1}}-\left(A_{\text {fluid }} K+A_{\text {solid }} K_{\text {solid }}\right) d T_{0} / d x \text { (A 35) }
$$

Taking the spatial average of $T_{1} \tilde{u}_{1}$, Equation (A 35) becomes

$$
\begin{aligned}
\dot{H}_{2}=\rho_{0} U_{a} h_{0} & +\frac{T_{0} \beta}{2} \operatorname{Re}\left[p_{1} \tilde{U}_{1}\left(1-\frac{\left(f_{x}-\tilde{f}_{v}\right)}{(1+\sigma)\left(1-\tilde{f}_{v}\right)}\right)\right] \\
& +\frac{\rho_{0} c_{p}\left|U_{1}\right|^{2}}{2 \omega A_{\text {fluid }}\left(1-\sigma^{2}\right)\left|1-f_{v}\right|^{2}} \operatorname{Im}\left[f_{x}+\tilde{\sigma}_{v}\right] \frac{d T_{0}}{d x} \\
& +\frac{\left(1-T_{0} \beta\right)}{2} \operatorname{Re}\left[p_{1} \tilde{U}_{1}\right]-\left(A_{\text {fluid }} K+A_{\text {solid }} K_{\text {solid }}\right) \frac{d T_{0}}{d x}
\end{aligned}
$$


Finally, the important results of this appendix are collected here rewritten in forms giving a system of six real equations and six variables: $\operatorname{Re}\left[p_{1}\right], \operatorname{Im}\left[p_{1}\right], \operatorname{Re}\left[U_{1}\right], \operatorname{Im}\left[U_{1}\right], U_{a}$, and $T_{0}$ :

$$
\begin{gathered}
\frac{d p_{1}}{d x}=-\frac{i \omega \rho_{0}}{A_{\text {fluid }}\left(1-f_{v}\right)} U_{1} \\
\frac{d U_{1}}{d x}=-\frac{i \omega A_{\text {fluid }}}{\rho_{0} a^{2}}\left[1+(\gamma-1) f_{\mathrm{\kappa}}\right] p_{1}+\frac{\beta\left(f_{\mathrm{x}}-f_{v}\right)}{\left(1-f_{v}\right)(1-\sigma)} \frac{d T_{0}}{d x} U_{1} \\
\frac{d U_{a}}{d x}=\beta \frac{d T_{0}}{d x} U_{a} \\
\frac{d T_{0}}{d x}=\left\{\dot{H}_{2}-\frac{1}{2} \operatorname{Re}\left[p_{1} \tilde{U}_{1}\left(1-\frac{T_{0} \beta\left(f_{\mathrm{x}}-\tilde{f}_{v}\right)}{(1+\sigma)\left(1-\tilde{f}_{v}\right)}\right)\right]-\rho_{0} U_{\alpha} h_{0}\right\} \div \\
\left\{\frac{\rho_{0} c_{p}\left|U_{1}\right|^{2}}{2 \omega A_{\text {fluid }}\left(1-\sigma^{2}\right)\left|1-f_{v}\right|^{2}} \operatorname{Im}\left[f_{\mathrm{x}}+\tilde{\sigma}_{v}\right]-A_{\text {fluid }} K-A_{\text {solid }} K_{\text {solid }}\right\}
\end{gathered}
$$

This equation set was used to modify the linear acoustic approximation model, DeltaE (Ward and Swift, 1997). The experimental data taken in this work and presented in Chapter 4 were used to validate this equation set as implemented in the DeltaE model modified for applied flow. 
APPENDIX B

REDUCED DATA 
Table B.1. Variables Listed in Reduced Data

\begin{tabular}{|c|c|}
\hline Quantity & Description \\
\hline$U_{a}$ & volumetric velocity per side \\
\hline $\begin{array}{l}f \\
\xi_{a}\end{array}$ & $\begin{array}{l}\text { operating frequency as indicated by waveform generator } \\
\text { dimensionless velocity through stack }\end{array}$ \\
\hline$p_{0}$ & mean pressure as measured at upper left elbow \\
\hline$T_{U}$ & temperature of gas at upper tee \\
\hline$T_{L}$ & temperature of gas at lower tee \\
\hline$\dot{W}_{\text {elec }}$ & electrical power supplied to left loudspeaker \\
\hline$\dot{W}_{a c}$ & acoustic power supplied to left loudspeaker \\
\hline$\dot{Q}_{w}$ & thermal power rejected by water flowing through left loudspeaker \\
\hline$\dot{W}_{\text {elec }}$ & electrical power supplied to right loudspeaker \\
\hline$\dot{W}_{a c}$ & acoustic power supplied to right loudspeaker \\
\hline$\dot{Q}_{w}$ & thermal power rejected by water flowing through right loudspeaker \\
\hline$\left|p_{1}\right|$ & magnitude of dynamic pressure measured in front of loudspeaker pair \\
\hline$T_{U}$ & gas temperature measured at upper elbow \\
\hline$\dot{Q}_{h x}$ & thermal power rejected by water cooling lines at hot heat exchanger \\
\hline$\dot{Q}_{H}$ & thermoacoustic power rejected to hot heat exchanger by Equation (74) \\
\hline$\dot{Q}_{H}$ & thermoacoustic power rejected to hot heat exchanger by heat balance \\
\hline$T_{1}$ & stack plate temperature measured $0.00 \mathrm{~cm}$ from hot end \\
\hline$T_{2}$ & stack plate temperature measured $1.69 \mathrm{~cm}$ from hot end \\
\hline$T_{3}$ & stack plate temperature measured $3.39 \mathrm{~cm}$ from hot end \\
\hline$T_{4}$ & stack plate temperature measured $5.08 \mathrm{~cm}$ from hot end \\
\hline$T_{5}$ & stack plate temperature measured $6.77 \mathrm{~cm}$ from hot end \\
\hline$T_{6}$ & stack plate temperature measured $8.47 \mathrm{~cm}$ from hot end \\
\hline$T_{7}$ & stack plate temperature measured $10.16 \mathrm{~cm}$ from hot end \\
\hline$T_{8}$ & stack plate temperature measured $11.85 \mathrm{~cm}$ from hot end \\
\hline$T_{9}$ & stack plate temperature measured $13.55 \mathrm{~cm}$ from hot end \\
\hline$T_{10}$ & stack plate temperature measured $15.24 \mathrm{~cm}$ from hot end \\
\hline$\Delta \dot{H}_{0}$ & change in flow enthalpy across stack \\
\hline$\dot{Q}_{C}$ & thermoacoustic power absorbed by stack from cold heat exchanger \\
\hline$\dot{Q}_{c x}$ & electrical power dissipated at cold heat exchanger \\
\hline$T_{L}$ & gas temperature measured at lower elbow \\
\hline
\end{tabular}


Table B.2 Variables Listed in System Performance Measures

\begin{tabular}{cl}
\hline \hline Quantity & Description \\
\hline$\Sigma_{\text {tot }}$ & numerator of Equation (73) \\
$\Sigma_{\text {elec }}$ & electrical power supplied to refrigerator \\
$\% \Delta_{\text {tot } e l e c}$ & Equation (73) \\
$\% \Delta_{\text {ac }}$ & side to side discrepancy in acoustic power supplied to the resonator \\
$\dot{W}_{\text {rev }}$ & $\dot{W}_{\text {rev }}=\dot{Q}_{H}\left(1-T_{C} / T_{H}\right)-\dot{m}\left(h_{C}-h_{H}\right)+\dot{m} T_{C}\left(s_{C}-s_{H}\right)$ \\
$\bar{\beta}_{2}$ & $\bar{\beta}_{2}=\left[\dot{Q}_{H}+\dot{m}\left(h_{C}-h_{H}\right)\right] / \dot{W}_{a c}$ \\
$\left(\bar{\beta}_{2} / \bar{\beta}_{2,0}\right)_{\text {actual }}$ & quantity described by Equation $(84)$ \\
& \\
$\left(\bar{\beta}_{2} / \bar{\beta}_{2,0}\right)_{\text {ideal }}$ & quantity described by Equation $(85)$ \\
$\bar{\varepsilon}_{2}$ & $\bar{\varepsilon}_{2}=\bar{\beta}_{2} / \bar{\beta}_{2, \text { Camot }}$ \\
$\dot{W}_{\text {rev }}$ & $\dot{W}_{\text {rev }}=\dot{Q}_{C}\left(1-T_{H} / T_{C}\right)-\dot{m}\left(h_{C}-h_{H}\right)+\dot{m} T_{H}\left(s_{C}-s_{H}\right)$ \\
$\beta_{1}$ & $\beta_{1}=\left[-\dot{Q}_{C}+\dot{m}\left(h_{C}-h_{H}\right)\right] / \dot{W}_{a c}$ \\
$\left(\beta_{1} / \beta_{1,0}\right)_{a c t u a l}$ & quantity described by Equation $(81)$ \\
$\left(\beta_{1} / \beta_{1,0}\right)_{\text {ideal }}$ & quantity described by Equation $(83)$ \\
$\varepsilon_{1}$ & $\varepsilon_{1}=\beta_{1} / \beta_{1, \text { camot }}$ \\
\hline
\end{tabular}


Table B.3. Enthalpy Flux Ratio Definitions

Designation General Definition Simplified Definition

$\mathbb{E}$

Global

$$
\bar{\Xi}_{\mathrm{G}}=\frac{\Delta \dot{H}_{0}}{\dot{H}_{2}^{H}}
$$

$$
\bar{\Xi}_{\mathrm{G}}=\frac{U_{a}\left(T_{H}-T_{C}\right)}{\dot{H}_{2}^{H} / \rho_{0} c_{p}}
$$

Short Stack

$$
\bar{\Xi}_{\mathrm{s}}=\frac{\dot{H}_{0}^{\text {ref }}}{\dot{H}_{2}^{\text {ref }}} \frac{\nabla T_{0} L}{T_{0}} \quad \bar{\Xi}_{\mathrm{S}}=\left(\frac{\gamma}{\gamma-1}\right)\left(\frac{p_{0}}{p_{1}^{s}}\right)\left(\frac{f L}{u_{1}^{s}}\right)\left\langle\xi_{a}\right)\left(\frac{\nabla T_{\text {ref }} \nabla T_{\text {crit }} L}{T_{\text {ref }}\left(\nabla T_{\text {ref }}-\nabla T_{\text {crit }}\right)}\right)\left(\frac{4 A_{\text {fluid }}}{\Pi \delta_{\kappa}}\right)
$$

Local

$$
\bar{\Xi}=\frac{\nabla \dot{H}_{0} L}{\dot{H}_{2}}
$$

$$
\bar{\Xi}=\frac{U_{a} \nabla T_{0} L}{\dot{H}_{2}^{H} / \rho_{0} c_{p}+U_{a}\left(T_{H}-T(x)\right)}
$$


Table B.4. Reduced Data on Left Side of Resonator

\begin{tabular}{|c|c|c|c|c|c|c|c|c|c|c|c|c|c|c|c|}
\hline thant & tato & Novitos & 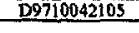 & & & & & & & & & & & & \\
\hline$v_{a}$ & Global & $-5.01 \times 10^{-3}$ & $-3.72 \times 10^{-3}$ & $-2.53 \times 10^{-3}$ & $-1.23 \times 10^{-3}$ & $0.00 \times 10^{0}$ & $6.24 \times 10^{-4}$ & $1.29 \times 10^{-3}$ & $1.95 \times 10^{-3}$ & $2.57 \times 10^{-3}$ & $3.22 \times 10^{-3}$ & $3.87 \times 10^{-3}$ & $4.49 \times 10^{-3}$ & $5.21 \times 10^{-3}$ & $\mathrm{~m}^{3}$ \\
\hline$f$ & Global & 93.938 & 93.938 & 93.938 & 93.938 & 93.938 & 93.938 & 93.938 & 93.938 & 93.938 & 93.938 & 93.938 & 93.938 & 93.938 & $\mathrm{~Hz}$ \\
\hline$\xi$ & Global & -0.02639 & -0.01959 & -0.01333 & -0.00645 & 0.00000 & 0.00329 & 0.00681 & 0.01026 & 0.01356 & 0.01693 & 0.02038 & 0.02363 & 0.02744 & - \\
\hline$p_{i}$ & Global & 321998 & 322566 & 322529 & 322369 & 322703 & 322438 & 322820 & 322778 & 323239 & 322345 & 322719 & 322982 & 322280 & $\mathrm{~Pa}$ \\
\hline$T_{v}$ & Global & 309.22 & 309.17 & 308.39 & 308.22 & 297.83 & 304.22 & 304.11 & 303.89 & 303.28 & 302.61 & 302.17 & 301.56 & 301.22 & K \\
\hline$T_{t}$ & Global & 302.22 & 301.89 & 297.17 & 297.44 & 298.67 & 297.11 & 296.72 & 296.39 & 294.61 & 294.22 & 294.50 & 301.28 & 298.56 & K \\
\hline$w_{r k r}$ & Pod 1 & -66.7 & -66.7 & -64.7 & -65.1 & -70.1 & -66.4 & -67.7 & -68.3 & -70.0 & -70.5 & -70.9 & -67.3 & -68.2 & $W_{e}$ \\
\hline$\dot{w}_{\alpha}$ & Pod I & -26.4 & -27.4 & -25.9 & -26.4 & -27.7 & -27.0 & -27.0 & -27.0 & -27.0 & -27.6 & -27.2 & -26.9 & $=26.9$ & $W_{a}$ \\
\hline$\dot{Q}_{n}$ & Pod: & -45.2 & -45.9 & -43.6 & -45.2 & -45.2 & -39.5 & -38.6 & -38.2 & -36.2 & -32.7 & -30.7 & -38.1 & -32.3 & $w_{1}$ \\
\hline$w_{s e c c}$ & Pod 2 & $.73,0$ & -73.1 & -70.9 & -70.9 & -71.1 & -72.7 & -73.7 & -74.3 & -76.1 & $-76,3$ & -76.3 & -73.4 & -74.5 & $W_{e}$ \\
\hline$\dot{w}_{a}$ & Pod 2 & -27.1 & -27.1 & -26.6 & -26.7 & -26.5 & -27.2 & -27.3 & -27.4 & -27.4 & .27 .3 & -27.3 & -27.2 & -27.2 & $W_{a}$ \\
\hline$\dot{Q}$ & Pod 2 & -48.5 & -49.2 & -47.5 & -48.9 & -44.8 & -43.2 & -39.6 & -40.8 & -38.2 & -34.3 & -34.6 & -40.2 & -35.7 & $W_{1}$ \\
\hline$\left|p_{1}\right|$ & Left & 6466 & 6451 & 6440 & 6450 & 6462 & 6458 & 6465 & 6479 & 6487 & 6466 & 6472 & 6461 & 6391 & $\mathrm{~Pa}$ \\
\hline $\boldsymbol{T}_{t^{\prime}}$ & Left & 309.39 & 309.11 & 308.39 & 307.89 & 308.22 & 307.56 & 306.33 & 305.50 & 303.78 & 303.17 & 302.39 & 304.61 & 302.72 & K \\
\hline$\dot{Q}_{n}$ & Left & -19.4 & -54.5 & -77.2 & -101.0 & -152.3 & -138.6 & -111.4 & -101.1 & -67.0 & -47.6 & -29.9 & -85.4 & -32.8 & $w_{t}$ \\
\hline$\dot{Q}_{H}$ & Left & -38.4 & -66.4 & -80.5 & -101.1 & -152.3 & -139.2 & -116.7 & -113.0 & -94.4 & -87.8 & -85.7 & -123.3 & -101.7 & $W_{1}$ \\
\hline$\dot{Q}_{t}$ & Lefl & -63.9 & -84.9 & -102.3 & -120.4 & -128.7 & -133.4 & -138.1 & -141.2 & -143.0 & -145.0 & -146.0 & -151.3 & -145.0 & $W_{1}$ \\
\hline$\tau_{1}$ & Left & 307.94 & 307.89 & 307.89 & 307.83 & 307.78 & 307.89 & 307.89 & 307.83 & 307.83 & 307.94 & 307.89 & 307.83 & 307.78 & K \\
\hline$r_{2}$ & Left & 306.00 & 306.39 & 306.89 & 307.33 & 307.33 & 307.61 & 307.78 & 307.83 & 308.00 & 308.22 & 308.17 & 308.22 & 308.39 & K \\
\hline$T_{3}$ & Left & 304.67 & 305.17 & 305.78 & 306.28 & 306.67 & 306,94 & 307.22 & 307.39 & 307.56 & 307.89 & 308.00 & 308.06 & 308.33 & K \\
\hline$T_{4}$ & Left & 303.61 & 304.44 & 304.89 & 305.44 & 306.11 & 306.39 & 306.56 & 306.72 & 307.00 & 307.33 & 307.56 & 307.67 & 308.00 & K \\
\hline$T_{s}$ & Left & 302.61 & 303.11 & 303.83 & 304.61 & 304.78 & 305.22 & 305.56 & 305.89 & 306.39 & 306.56 & 306.83 & 307.11 & 307.61 & K \\
\hline$T_{i}$ & Left & 302.00 & 302,33 & 303.00 & 303.56 & 303.94 & 304.39 & 304.89 & 305.00 & 305.22 & 305.67 & 306.00 & 306.39 & 307.06 & K \\
\hline$T$ & Left & 301.44 & 301.72 & 302.33 & 302.67 & 303.06 & 303.39 & 303.72 & 304.00 & 304.44 & 304.67 & 305.00 & 305.78 & 306.28 & K \\
\hline$r_{x}$ & Left & 301.22 & 301.39 & 1.72 & 302.00 & 302.50 & 302.50 & 302.94 & 302.89 & 303.11 & 303.56 & 303.83 & 304.39 & 305.28 & K \\
\hline$r_{q}$ & Left & .33 & 300.22 & 0.56 & 300.67 & 300.72 & 300.89 & 301.11 & 301.22 & .22 & 301.61 & 301.72 & 302.44 & 303.50 & K \\
\hline$T_{\mathrm{ig}}$ & Left & 300.72 & 300.44 & 300.61 & 300.50 & 300.61 & 300.56 & 300.56 & 300.61 & 300.50 & 300.67 & 300.67 & 301.33 & 302.39 & K \\
\hline$\Delta \dot{H}_{11}$ & Left & -94.6 & -72.5 & -48.2 & -23.5 & 0.0 & 12.0 & 24.9 & 36.9 & 49.6 & 61.2 & 73.3 & 76.5 & 73.5 & $w_{1}$ \\
\hline$\dot{Q}_{r}$ & Left & 105.1 & 102.8 & 98.1 & 90.8 & 74.6 & 67.3 & 59.0 & 50.0 & 39.2 & 28.8 & 18.4 & 20.9 & 17.4 & $W_{1}$ \\
\hline$\dot{e}$ & Left & 80.4 & 83.9 & 110.3 & 90.5 & 74.6 & 69.7 & 62.9 & 53.7 & 40.0 & 21.3 & 2.6 & 0.0 & 0.0 & $W_{e}$ \\
\hline$T_{L}$ & Left & 302.61 & 302.39 & 298.78 & 300.61 & 302.78 & 302.00 & 301.72 & 301.33 & 300.61 & 299.78 & 299.11 & 299.56 & 301.11 & $\mathbf{K}$ \\
\hline
\end{tabular}


Table B.5. Reduced Data on Right Side of Resonator

\begin{tabular}{|c|c|c|c|c|c|c|c|c|c|c|c|c|c|c|c|}
\hline Quantity & atily Lo & D9710041628 & D9710042103 & D9710041153 & D9710051215 & D9709261444 & D9710011646 & D9709301540 & D971001212 & D970930120 & 09710021545 & D9709301915 & D9710010008 & D9709291730 & Unit \\
\hline$v_{a}$ & Global & $-5.01 \times 10^{-3}$ & $-3.72 \times 10^{-3}$ & $-2.53 \times 10^{-3}$ & $-1.23 \times 10^{-3}$ & $0.00 \times 10^{0}$ & $6.24 \times 10^{-4}$ & $1.29 \times 10^{-3}$ & $1.95 \times 10^{-3}$ & $2.57 \times 10^{-3}$ & $3.22 \times 10^{-3}$ & $3.87 \times 10^{-3}$ & $4.49 \times 10^{-3}$ & $5.21 \times 10^{-3}$ & $\mathrm{~m}^{3}$ \\
\hline$f$ & Global & 93.938 & 93.938 & 93.938 & 93.938 & 93.938 & 93.938 & 93.938 & 93.938 & 93.938 & 93.938 & 93.938 & 93.938 & 93.938 & $\mathrm{~Hz}$ \\
\hline$\xi$ & Global & -0.0246 & -0.0183 & -0.0124 & -0.0060 & 0.0000 & 0.0031 & 0.0063 & 0.0096 & 0.0126 & 0.0158 & 0.0190 & 0.0220 & 0.0256 & - \\
\hline$p_{0}$ & Global & 321998 & 322566 & 322529 & 322369 & 322703 & 322438 & 322820 & 322778 & 323239 & 322345 & 322719 & 322982 & 322280 & $\mathrm{~Pa}$ \\
\hline$\tau_{v}$ & Global & 309.22 & 309.17 & 308.39 & 308.22 & 297.83 & 304.22 & 304.11 & 303.89 & 303.28 & 302.61 & 302.17 & 301.56 & 301.22 & K \\
\hline$T_{L}$ & Global & 302.22 & 301.89 & 297.17 & 297.44 & 298.67 & 297.11 & 296.72 & 296.39 & 294.61 & 294.22 & 294.50 & 301.28 & 298.56 & $\mathrm{~K}$ \\
\hline$W_{r k e}$ & Pod 3 & -75.8 & -75.9 & -73.7 & -74.4 & -73.5 & -75.7 & -76.5 & -77.3 & -78.9 & -79.1 & -79.6 & -76.0 & -77.8 & $W_{e}$ \\
\hline$\dot{w}$ & Pod 3 & -27.6 & -27.3 & -26.6 & -27.0 & -27.1 & -27.2 & -27.2 & -27.5 & -27.4 & -27.3 & -27.1 & -26.6 & -26.3 & $\mathrm{w}_{\mathrm{a}}$ \\
\hline$\dot{Q}_{n}$ & Pod 3 & -52.8 & -50.7 & -49.9 & -52.0 & -46.5 & -49.0 & -43.3 & -46.5 & -41.7 & -37.5 & -36.4 & -43.3 & -39.5 & $w_{t}$ \\
\hline$\dot{w}_{\mathrm{rkc}}$ & Pod 4 & -76.4 & -76.6 & -74.3 & -75.1 & -75.7 & -76.5 & -77.3 & -78.0 & -79.8 & -79.8 & -80.5 & -76.9 & -78.1 & $W_{e}$ \\
\hline$\dot{w}_{x}$ & Pod 4 & -27.6 & -27.6 & -26.9 & -27.0 & -27.5 & -27.2 & -27.1 & -27.3 & -27.2 & -26.9 & -27.3 & -27.1 & -26.8 & $W_{\mathrm{a}}$ \\
\hline$\dot{\mathbf{Q}}_{i r}$ & Pod 4 & -52.5 & -51.4 & -48.1 & -49.2 & -49.5 & -45.1 & -40.2 & -43.0 & -39.6 & -35.4 & -36.7 & -42.4 & -36.2 & $w_{t}$ \\
\hline$\left|p_{1}\right|$ & Right & 6429 & 6436 & 6425 & 6429 & 6428 & 6430 & 6433 & 6434 & 6450 & 6429 & 6448 & 6456 & 6445 & $\mathbf{P a}$ \\
\hline$T_{u}$ & Right & 309.11 & 308.83 & 308.11 & 307.94 & 308.39 & 307.67 & 306.00 & 304.83 & 302.22 & 301.06 & 300.67 & 304.00 & 301.61 & $\mathrm{~K}$ \\
\hline$\dot{\boldsymbol{Q}}_{k}$ & Righs & -57.5 & -77.6 & -93.0 & -100.9 & -122.4 & -123.6 & -115.7 & -99.6 & -85.0 & -71.9 & -58.2 & -88.5 & -52.7 & $W_{1}$ \\
\hline$\dot{Q}_{H}$ & Right & -73.5 & -87.3 & -94.5 & -101.1 & -122.4 & -123.9 & -122.1 & -115.2 & -122.9 & -128.9 & -130.3 & -134.3 & $-138,3$ & $W_{t}$ \\
\hline$\dot{\theta}_{H}$ & Right & -87.8 & -106.1 & -119.9 & -133.3 & -141.4 & -146.4 & -149.1 & -152.4 & -155.1 & -154.0 & -153.7 & -156.7 & -156.2 & $W_{t}$ \\
\hline$T_{1}$ & Right & 307.89 & 307.83 & 307.89 & 307.89 & 307.89 & 307.83 & 307.89 & 307.89 & 307.83 & 307.83 & 307.78 & 307.89 & 307.89 & K \\
\hline$T_{2}$ & Right & 306.28 & 306,50 & 306.83 & 307.17 & 307.33 & 307.44 & 307.56 & 307.67 & 307.72 & 307.83 & 307.72 & 308.00 & 308.00 & K \\
\hline$\tau_{s}$ & Right & 304.61 & 305.11 & 305.72 & 306.28 & 306.50 & 306.78 & 307.11 & 307.39 & 307.39 & 307.67 & 307.67 & 307.89 & 308.00 & K \\
\hline$T_{4}$ & Right & 303.56 & 304.11 & 304.67 & 305.17 & 305,94 & 305.94 & 306.50 & 306.61 & 306.89 & 307,06 & 307.22 & 307.50 & 307.61 & K \\
\hline$T_{4}$ & Right & 302.56 & 302.94 & 303.56 & 304.39 & 304.67 & 304.94 & 305.44 & 305.72 & 306.00 & 306.28 & 306.44 & 306.89 & 307.11 & $\mathrm{~K}$ \\
\hline$T_{\mathrm{h}}$ & Righth & 301.83 & 302.11 & 302.72 & 303.17 & 303.67 & 304.00 & 304.44 & 304.78 & 305.00 & 305.44 & 305.67 & 306.06 & 306.56 & $\mathrm{~K}$ \\
\hline$T_{7}$ & Right & 301.39 & 301.56 & 302.06 & 302.50 & 302.94 & 303.22 & 303.61 & 303.94 & 304.33 & 304.56 & 304.83 & 305.17 & 305.44 & $\mathrm{~K}$ \\
\hline$T_{\mathrm{k}}$ & Right & 300.61 & 300.67 & 301.06 & 301.22 & 301.56 & 301.72 & 302.11 & 302.39 & 302.56 & 302.83 & 303.06 & 303.44 & 303.67 & $\mathbf{K}$ \\
\hline$T_{q}$ & Right & 300.72 & 300.72 & 300.94 & 301.11 & 301.28 & 301.33 & 301,67 & 301.78 & 301.94 & 302.00 & 302.17 & 302.33 & 302.56 & $\mathrm{~K}$ \\
\hline$T_{10}$ & Right & 300.56 & 300.50 & 300.67 & 300.61 & 300.50 & 300.50 & 300.56 & 300.61 & 300.50 & 300.56 & 300.50 & 300.61 & 300.56 & $\mathrm{~K}$ \\
\hline$\Delta \dot{H}_{n}$ & Right & -96.1 & -71.5 & -47.9 & -23.3 & 0.0 & 12.0 & 24.9 & 37.2 & 49.6 & 61.3 & 73.8 & 85.6 & 100.0 & $W_{t}$ \\
\hline$\dot{Q}_{c}$ & Right & 128.8 & 122.5 & 114.3 & 102.6 & 86.8 & 79.9 & 70.0 & 60.5 & 51.1 & 38.6 & 25.7 & 17.5 & 3.2 & $w_{t}$ \\
\hline$\dot{Q}_{x}$ & Right & 100.4 & 102.0 & 126.1 & 101.5 & 86.8 & 89.1 & 90.1 & 91.5 & 89.4 & 83.9 & 74.2 & 56.1 & 19.1 & $W_{\mathrm{e}}$ \\
\hline$T_{L}$ & Right & 302.72 & 302.61 & 298.89 & 300.94 & 305.50 & 306.11 & 306.50 & 306.67 & 306.17 & 305.94 & 305.28 & 303.89 & 301.72 & K \\
\hline
\end{tabular}


Table B.6. Estimated Uncertainty for Left Side Reduced Data

\begin{tabular}{|c|c|c|c|c|c|c|c|c|c|c|c|c|c|c|c|}
\hline Quantity & Location & D9710041628 & D99710042105 & D9710041153 & D9710051215 & D9709261444 & D9710011646 & D99709301540 & D9710012125 & D9709301200 & D9710021545 & D9709301915 & D9710010008 & D9709291730 & Unit \\
\hline$U_{\mathrm{a}}$ & Global & $6.64 \times 10^{-5}$ & $5.05 \times 10^{-5}$ & $3.69 \times 10^{-5}$ & $2.34 \times 10^{-5}$ & $1.71 \times 10^{-5}$ & $1.92 \times 10^{-5}$ & $2.42 \times 10^{-5}$ & $3.06 \times 10^{-5}$ & $3.77 \times 10^{-5}$ & $4.52 \times 10^{-5}$ & $5.31 \times 10^{-5}$ & $6.00 \times 10^{-5}$ & $6.94 \times 10^{-5}$ & $\mathrm{~m}^{3}$ \\
\hline$f$ & Globas & $3 \times 10^{-3}$ & $3 \times 10^{3}$ & $3 \times 10^{-3}$ & $3 \times 10^{-3}$ & $3 \times 10^{-3}$ & $3 \times 10^{-3}$ & $3 \times 10^{-3}$ & $3 \times 10^{-3}$ & $3 \times 10^{-3}$ & $3 \times 10^{-3}$ & $3 \times 10^{-3}$ & $3 \times 10^{-3}$ & $3 \times 10^{-3}$ & $\mathrm{~Hz}$ \\
\hline$\xi$ & Global & $3.50 \times 10^{-4}$ & $2.66 \times 10^{-4}$ & $1.94 \times 10^{-4}$ & $1.23 \times 10^{-4}$ & $9.00 \times 10^{-5}$ & $1.01 \times 10^{-4}$ & $1.27 \times 10^{-4}$ & $1.61 \times 10^{-4}$ & $1.98 \times 10^{-4}$ & $2,38 \times 10^{-4}$ & $2.80 \times 10^{-4}$ & $3.16 \times 10^{-4}$ & $3.65 \times 10^{-4}$ & - \\
\hline$P_{w}$ & Global & 653 & 653 & 653 & 653 & 653 & 653 & 653 & 653 & 653 & 653 & 653 & 653 & 653 & $\mathrm{~Pa}$ \\
\hline$T_{u}$ & Global & 2.2 & 2.2 & 2.2 & 2.2 & 2.2 & 2.2 & 2.2 & 2.2 & 2.2 & 2.2 & 2.2 & 2.2 & 2.2 & $\mathrm{~K}$ \\
\hline$T_{L}$ & Global & 2.2 & 2.2 & 2.2 & 2.2 & 2.2 & 2.2 & 2.2 & 2.2 & 2.2 & 2.2 & 2.2 & 2.2 & 2.2 & K \\
\hline$\dot{W}_{\mathrm{rk}}$ & Pod 1 & 2.70 & 2.70 & 2.62 & 2.63 & 2.84 & 2.69 & 2.74 & 2.76 & 2.83 & 2.85 & 2.87 & 2.72 & 2.76 & $W_{e}$ \\
\hline$\dot{w}_{\alpha}$ & Pod I & 2.56 & 2.58 & 2.50 & 2.52 & 2.59 & 2.53 & 2.51 & 2.51 & 2.49 & 2.51 & 2.48 & 2.51 & 2.47 & $\mathrm{~W}_{\mathrm{a}}$ \\
\hline$\dot{Q}_{w}$ & Pod 1 & 1.57 & 1.58 & 1.55 & 1.57 & 1.57 & 1.49 & 1.48 & 1.47 & 1.45 & 1.39 & 1.38 & 1.48 & 1.40 & $W_{1}$ \\
\hline$\dot{w}_{\mathrm{ret}}$ & Pod 2 & 2.95 & 2.96 & 2.87 & 2.87 & 2.88 & 2.94 & 2.98 & 3.01 & 3.08 & 3.09 & 3.09 & 2.97 & 3.02 & $W_{0}$ \\
\hline$\dot{w}_{\boldsymbol{\alpha}}$ & $\operatorname{Pod} 2$ & 2.58 & 2.57 & 2.52 & 2.53 & 2.56 & 2.53 & 2.51 & 2.52 & 2.50 & 2.49 & 2.48 & 2.51 & 2.46 & $w_{a}$ \\
\hline$\dot{Q}$ & Pod 2 & 1.63 & 1.64 & 1.61 & 1.62 & 1.57 & 1.54 & 1.48 & 1.50 & 1.47 & 1.39 & 1.41 & 1.49 & 1.44 & $W_{t}$ \\
\hline$\left|p_{1}\right|$ & Left & 195 & 194 & 194 & 194 & 195 & 195 & 195 & 195 & 195 & 195 & 195 & 195 & 193 & $\mathrm{~Pa}$ \\
\hline$r_{v}$ & Left & 2.2 & 2.2 & 2.2 & 2.2 & 2.2 & 2.2 & 2.2 & 2.2 & 2.2 & 2.2 & 2.2 & 2.2 & 2.2 & $\mathrm{~K}$ \\
\hline$\dot{Q}_{\mathrm{nt}}$ & Left & 5.05 & 5.26 & 5.31 & 4.60 & 3.45 & 3.29 & 3.21 & 3.37 & 5.00 & 5.17 & 4.87 & 3.57 & 5.02 & $W_{1}$ \\
\hline$\dot{e}_{n}$ & Left & 5.35 & 5.42 & 5.38 & 4.62 & 3.45 & 3.30 & 3.24 & 3.44 & 5.08 & 5.29 & 5.05 & 3.87 & 5.27 & $w_{1}$ \\
\hline$T_{1}$ & Leff & 0.5 & 0.5 & 0.5 & 0.5 & 0.5 & 0.5 & 0.5 & 0.5 & 0.5 & 0.5 & 0.5 & 0.5 & 0.5 & K \\
\hline$T_{2}$ & Left & 0.5 & 0.5 & 0.5 & 0.5 & 0.5 & 0.5 & 0.5 & 0.5 & 0.5 & 0.5 & 0.5 & 0.5 & 0.5 & $\mathrm{~K}$ \\
\hline$T_{3}$ & Left & 0.5 & 0.5 & 0.5 & 0.5 & 0.5 & 0.5 & 0.5 & 0.5 & 0.5 & 0.5 & 0.5 & 0.5 & 0.5 & K \\
\hline$T_{4}$ & Left & 0.5 & 0.5 & 0.5 & 0.5 & 0.5 & 0.5 & 0.5 & 0.5 & 0.5 & 0.5 & 0.5 & 0.5 & 0.5 & K \\
\hline$T_{3}$ & Left & 0.5 & 0.5 & 0.5 & 0.5 & 0.5 & 0.5 & 0.5 & 0.5 & 0.5 & 0.5 & 0.5 & 0.5 & 0.5 & K \\
\hline$T_{A}$ & Left & 0.5 & 0.5 & 0.5 & 0.5 & 0.5 & 0.5 & 0.5 & 0.5 & 0.5 & 0.5 & 0.5 & 0.5 & 0.5 & K \\
\hline$T_{7}$ & Left & 0.5 & 0.5 & 0.5 & 0.5 & 0.5 & 0.5 & 0.5 & 0.5 & 0.5 & 0.5 & 0.5 & 0.5 & 0.5 & K \\
\hline$T_{n}$ & Left & 0.5 & 0.5 & 0.5 & 0.5 & 0.5 & 0.5 & 0.5 & 0.5 & 0.5 & 0.5 & 0.5 & 0.5 & 0.5 & K \\
\hline$T_{y}$ & Left & 0.5 & 0.5 & 0.5 & 0.5 & 0.5 & 0.5 & 0.5 & 0.5 & 0.5 & 0.5 & 0.5 & 0.5 & 0.5 & K \\
\hline$T_{\mathrm{ttI}}$ & Lefl & 0.5 & 0.5 & 0.5 & 0.5 & 0.5 & 0.5 & 0.5 & 0.5 & 0.5 & 0.5 & 0.5 & 0.5 & 0.5 & K \\
\hline$\Delta \dot{H}_{a}$ & Left & 1.76 & 1.32 & 0.89 & 0.43 & 0.00 & 0.22 & 0.45 & 0.68 & 0.90 & 1.12 & 1.34 & 1.49 & 1.62 & $W_{1}$ \\
\hline 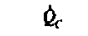 & Lef & 3.74 & 3.67 & 4.40 & 3.67 & 3.11 & 2.95 & 2.74 & 2.47 & 2.09 & 1.62 & 1.37 & 1.49 & 1.62 & $W_{1}$ \\
\hline$\dot{\theta}_{*}$ & Left & 3.31 & 3.42 & 4.31 & 3.65 & 3.11 & 2.94 & 2.70 & 2.38 & 1.88 & 1.17 & 0.29 & 0.00 & 0.00 & $W_{e}$ \\
\hline$T_{L}$ & Left & 2.2 & 2.2 & 2.2 & 2.2 & 2.2 & 2.2 & 2.2 & 2.2 & 2.2 & 2.2 & 2.2 & 2.2 & 2.2 & K \\
\hline
\end{tabular}


Table B.7. Estimated Uncertainty for Right Side Reduced Data

\begin{tabular}{|c|c|c|c|c|c|c|c|c|c|c|c|c|c|c|c|}
\hline ant & Ation & & 10042105 & 53 & $20001<1$ & 1144 & 10011040 & 9301540 & $\pi$ & 1200 & 71002154 & 11915 & 10008 & 30 & Unil \\
\hline$v_{a}$ & Global & $6.64 \times 10^{-5}$ & $5.05 \times 10^{-5}$ & $3.69 \times 10^{-5}$ & $2.34 \times 10^{-5}$ & $1.71 \times 10^{-5}$ & $1.92 \times 10^{-5}$ & $2.42 \times 10^{-5}$ & $3.06 \times 10^{-5}$ & $3.77 \times 10^{-5}$ & $4.52 \times 10^{-5}$ & $5.31 \times 10^{-5}$ & $6.00 \times 10^{-5}$ & $6.94 \times 10^{-5}$ & $\mathrm{~m}^{3}$ \\
\hline$f$ & Global & $3 \times 10^{-3}$ & $3 \times 10^{-3}$ & $3 \times 10^{-3}$ & $3 \times 10^{-3}$ & $3 \times 10^{-3}$ & $3 \times 10^{-3}$ & $3 \times 10^{-3}$ & $3 \times 10^{-3}$ & $3 \times 10^{-3}$ & $3 \times 10^{-3}$ & $3 \times 10^{-3}$ & $3 \times 10^{-3}$ & $3 \times 10^{-3}$ & $\mathrm{~Hz}$ \\
\hline$\xi_{\infty}$ & Global & $3.50 \times 10^{-4}$ & $2.66 \times 10^{-4}$ & $1.94 \times 10^{-4}$ & $1.23 \times 10^{-4}$ & $9.00 \times 10^{-5}$ & $1.01 \times 10^{-4}$ & $1.27 \times 10^{-4}$ & $1.61 \times 10^{-4}$ & $1.98 \times 10^{-4}$ & $2.38 \times 10^{-4}$ & $2.80 \times 10^{-4}$ & $3.16 \times 10^{-4}$ & $3.65 \times 10^{-4}$ & - \\
\hline$p_{\mathrm{a}}$ & Global & 653 & 653 & 653 & 653 & 653 & 653 & 653 & 653 & 653 & 653 & 653 & 653 & 653 & $\mathrm{~Pa}$ \\
\hline$T_{t}$ & Global & 2.2 & 2.2 & 2.2 & 2.2 & 2.2 & 2.2 & 2.2 & 2.2 & 2.2 & 2,2 & 2.2 & 2.2 & 2.2 & $\mathrm{~K}$ \\
\hline$T_{t}$ & Olobal & 2.2 & 2.2 & 2.2 & 2.2 & 2.2 & 2.2 & 2.2 & 2.2 & 2.2 & 2.2 & 2.2 & 2.2 & 2.2 & $\mathrm{~K}$ \\
\hline$\dot{W}_{c k s}$ & $\operatorname{Pod} 3$ & 3.07 & 3.07 & 2,98 & 3.01 & 2.97 & 3.06 & 3.09 & 3.13 & 3.19 & 3.20 & 3.22 & 3.08 & 3.15 & $w_{e}$ \\
\hline$\dot{w}_{\alpha}$ & $\operatorname{Pod} 3$ & 2.55 & 2.54 & 2.48 & 2.50 & 2.52 & 2.49 & 2.47 & 2.47 & 2.45 & 2.44 & 2.43 & 2.46 & 2.43 & $W_{a}$ \\
\hline$\dot{Q}_{m}$ & Pod 3 & 1.69 & 1.66 & 1.64 & 1.68 & 1.59 & 1.63 & 1.54 & 1.59 & 1.52 & 1.45 & 1.44 & 1.54 & 1.49 & $W_{i}$ \\
\hline$\dot{w}_{\text {tetr }}$ & Pod 4 & 3.09 & 3.10 & 3.01 & 3.04 & 3.06 & 3.09 & 3.13 & 3.16 & 3.23 & 3.23 & 3.26 & 3.11 & 3.16 & $w_{e}$ \\
\hline$\dot{w_{\alpha}}$ & $\operatorname{Pod} 4$ & 2.52 & 2.52 & 2.46 & 2.48 & 2.51 & 2.46 & 2.44 & 2.44 & 2.42 & 2.41 & 2.41 & 2.45 & 2.41 & $w_{a}$ \\
\hline$\hat{Q}_{w}$ & $\operatorname{Pod} 4$ & 1.79 & 1.77 & 1.67 & 1.70 & 1.63 & 1.62 & 1.50 & 1.57 & 1.48 & 1.42 & 1.43 & 1.54 & 1.42 & $W_{1}$ \\
\hline$\left|p_{1}\right|$ & Right & 192 & 192 & 191 & 192 & 191 & 192 & 192 & 192 & 192 & 192 & 192 & 192 & 192 & $\mathrm{~Pa}$ \\
\hline$T_{v}$ & Right & 2.2 & 2.2 & 2.2 & 2.2 & 2.2 & 2.2 & 2.2 & 2.2 & 2.2 & 2.2 & 2.2 & 2.2 & 2.2 & K \\
\hline$\dot{e}_{n}$ & Right & 5.17 & 4.38 & 4.39 & 3.98 & 8.40 & 7.05 & 7.73 & 2.82 & 3.50 & 3.70 & 3.50 & 2.92 & 4.31 & $W_{1}$ \\
\hline$\dot{Q}_{t}$ & Right & 5.46 & 4.57 & 4.48 & 4.01 & 8.40 & 7.05 & 7.74 & 2.90 & 3.61 & 3.86 & 3.75 & 3.31 & 4.67 & $w_{1}$ \\
\hline$T_{1}$ & Right & 0.5 & 0.5 & 0.5 & 0.5 & 0.5 & 0.5 & 0.5 & 0.5 & 0.5 & 0.5 & 0.5 & 0.5 & 0.5 & K \\
\hline$T_{2}$ & Right & 0.5 & 0.5 & 0.5 & 0.5 & 0.5 & 0.5 & 0.5 & 0.5 & 0.5 & 0.5 & 0.5 & 0.5 & 0.5 & $\mathbf{K}$ \\
\hline$T_{3}$ & Right & 0.5 & 0.5 & 0.5 & 0.5 & 0.5 & 0.5 & 0.5 & 0.5 & 0.5 & 0.5 & 0.5 & 0.5 & 0.5 & $\mathbf{K}$ \\
\hline$T_{A}$ & Right & 0.5 & 0.5 & 0.5 & 0.5 & 0.5 & 0.5 & 0.5 & 0.5 & 0.5 & 0.5 & 0.5 & 0.5 & 0.5 & K \\
\hline$T_{a}$ & Right & 0.5 & 0.5 & 0.5 & 0.5 & 0.5 & 0.5 & 0.5 & 0.5 & 0.5 & 0.5 & 0.5 & 0.5 & 0.5 & $\mathbf{K}$ \\
\hline$T_{n}$ & Right & 0.5 & 0.5 & 0.5 & 0.5 & 0.5 & 0.5 & 0.5 & 0.5 & 0.5 & 0.5 & 0.5 & 0.5 & 0.5 & $\mathbf{K}$ \\
\hline$T_{i}$ & Right & 0.5 & 0.5 & 0.5 & 0.5 & 0.5 & 0.5 & 0.5 & 0.5 & 0.5 & 0.5 & 0.5 & 0.5 & 0.5 & K \\
\hline$T_{\mathrm{a}}$ & Right & 0.5 & 0.5 & 0.5 & 0.5 & 0.5 & 0.5 & 0.5 & 0.5 & 0.5 & 0.5 & 0.5 & 0.5 & 0.5 & K \\
\hline$T_{0}$ & Right & 0.5 & 0.5 & 0.5 & 0.5 & 0.5 & 0.5 & 0.5 & 0.5 & 0.5 & 0.5 & 0.5 & 0.5 & 0.5 & K \\
\hline$\tau_{10}$ & Right & 0.5 & 0.5 & 0.5 & 0.5 & 0.5 & 0.5 & 0.5 & 0.5 & 0.5 & 0.5 & 0.5 & 0.5 & 0.5 & K \\
\hline$\Delta \dot{H}_{1}$ & Right & 1.77 & 1.31 & 0.88 & 0.43 & 0.00 & 0.22 & 0.45 & 0.68 & 0.90 & 1.12 & 1.34 & 1.56 & 1.81 & $W_{1}$ \\
\hline$\dot{\theta}_{r}$ & Right & 2.55 & 2.28 & 2.44 & 1.90 & 1.60 & 1.65 & 1.72 & 1.81 & 1.88 & 1.91 & 1.93 & 1.89 & 1.85 & $w_{1}$ \\
\hline$\dot{Q}_{x}$ & Right & 1.83 & 1.86 & 2.27 & 1.85 & 1.60 & 1.64 & 1.66 & 1.68 & 1.65 & 1.55 & 1.38 & 1.07 & 0.41 & $W_{e}$ \\
\hline$T_{L}$ & Right & 2.2 & 2.2 & 2.2 & 2.2 & 2.2 & 2.2 & 2.2 & 2.2 & 2.2 & 2.2 & 2.2 & 2.2 & 2.2 & $k$ \\
\hline
\end{tabular}


Table B.8. System Performance Measures

\begin{tabular}{|c|c|c|c|c|c|c|c|c|c|c|c|c|c|c|c|}
\hline Quantity & Location & D9710041628 & D9710042105 & D9710041153 & D9710051215 & D9709261444 & D9710011646 & D9709301540 & D9710012125 & D9709301200 & D9710021545 & 09709301915 & D9710010008 & D9709291730 & Unit \\
\hline$\Sigma_{s s}$ & Left & 15.21 & 3.21 & 3.24 & -3.16 & -26.49 & -24.18 & -10.40 & -22.06 & -13.97 & -17.09 & -23.22 & -26.29 & 5.60 & $w$ \\
\hline $\boldsymbol{\Sigma}_{r k w}$ & Left & 128.36 & 152.74 & 171.55 & 191.91 & 215.79 & 197.13 & 179.25 & 158.04 & 127.44 & 97.47 & 72.05 & 137.38 & 106.34 & $w$ \\
\hline $\mathscr{F u}_{\text {witit }}$ & Left & $11.85 \%$ & $2.10 \%$ & $1.89 \%$ & $-1.65 \%$ & $-12.27 \%$ & $-12.26 \%$ & $-5.80 \%$ & $-13.96 \%$ & $-10,96 \%$ & $-17.54 \%$ & $-32.23 \%$ & $-19.14 \%$ & $5.26 \%$ & $\%$ \\
\hline $\mathscr{F s}_{a r}$ & Left & $-2.57 \%$ & $0.87 \%$ & $-2.79 \%$ & $-1.13 \%$ & $4.27 \%$ & $-0.73 \%$ & $-0.80 \%$ & $-1.33 \%$ & $-1.61 \%$ & $1.12 \%$ & $-0.37 \%$ & $-1.10 \%$ & $-0.97 \%$ & $\%$ \\
\hline$\dot{W}_{n}$ & Left & -2.62 & -2.94 & -2.99 & -3.15 & -3.00 & - & - & - & - & - & - & - & - & $w$ \\
\hline$\tilde{\beta}_{2}$ & Len & 2.96 & 2.89 & 2.87 & 2.71 & 2.38 & - & - & - & - & - & - & - & . & - \\
\hline$\left|B_{2} / \bar{B}_{r 0}\right|_{m+t}$ & Left & 1.25 & 1.21 & 1.21 & 1.14 & 1.00 & - & - & . & - & . & - & - & . & - \\
\hline 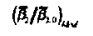 & Lef & 1.75 & 1.43 & 1.24 & 1.10 & 1.00 & - & - & - & - & - & - & . & - & - \\
\hline $\bar{\varepsilon}_{2}$ & Lef & $4.89 \%$ & $5.38 \%$ & $5.71 \%$ & $5.94 \%$ & $5.54 \%$ & - & - & . & - & - & - & - & - & - \\
\hline$\dot{w}_{\mathrm{mr}}$ & Left & - & - & - & - & -1.78 & -1.79 & -1.74 & -1.64 & -1.56 & -1.43 & -1.31 & - & . & w \\
\hline$\beta_{1}$ & Left & - & - & - & - & 1.38 & 1.46 & 1.54 & 1.60 & 1.63 & 1.64 & 1.68 & - & - & - \\
\hline$(\beta, \beta, \ldots, \ldots$ & Lef & - & - & - & - & 1.00 & 1.06 & 1.12 & 1.16 & 1.18 & 1.19 & 1.22 & - & - & - \\
\hline$\left.\left(\beta_{1} / \beta_{n}\right)_{1}\right)_{2 x}$ & Len & - & - & - & - & 1.00 & 1.16 & 1.30 & 1.43 & 1.57 & 1.69 & 1.81 & - & . & - \\
\hline$\varepsilon_{1}$ & Left & - & - & - & - & $3.28 \%$ & $3.30 \%$ & $3.20 \%$ & $3.02 \%$ & $2.86 \%$ & $2.61 \%$ & $2.41 \%$ & - & . & - \\
\hline$\Sigma_{v i}$ & Right & -1.8 & 3.8 & 8.7 & 14.5 & 17.7 & 11.9 & 19.6 & 19.4 & 23.2 & 27.4 & 25.2 & 31.5 & 10.2 & w \\
\hline$\Sigma_{r k r}$ & Right & 161.0 & 183.6 & 199.7 & 216.5 & 236.0 & 229.6 & 218.8 & 208.5 & 189.6 & 172.2 & 156.4 & 205.7 & 138.6 & $w$ \\
\hline$\% \Delta_{\text {ewhir }}$ & Right & $-1.13 \%$ & $2.09 \%$ & $4.36 \%$ & $6.69 \%$ & $7.50 \%$ & $5.20 \%$ & $8.97 \%$ & $9.30 \%$ & $12.26 \%$ & $15.94 \%$ & $16.09 \%$ & $15.30 \%$ & $7.35 \%$ & $\%$ \\
\hline$\% \Delta_{\alpha}$ & Right & $-0.19 \%$ & $-0.87 \%$ & $-1.06 \%$ & $-0.10 \%$ & $-1.62 \%$ & $0.06 \%$ & $0.52 \%$ & $0.71 \%$ & $0.63 \%$ & $1.34 \%$ & $-0.82 \%$ & $-1.72 \%$ & $-1.70 \%$ & $\%$ \\
\hline$w_{m}$ & Right & -3.25 & -3.38 & -3.38 & -3.43 & -3.39 & - & 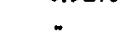 & . & - & - & - & - & - & w \\
\hline$\tilde{\beta}_{2}$ & Right & 3.33 & 3.23 & 3.14 & 2.90 & 2.59 & - & - & - & - & - & - & . & - & - \\
\hline$\left(B_{0} / B_{-1}\right)_{\text {rat }}$ & Right & 1.29 & 1.25 & 1.21 & 1.12 & 1.00 & - & - & - & - & - & - & - & - & - \\
\hline 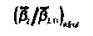 & Right & 1.55 & 1.34 & 1.20 & 1.09 & 1.00 & - & - & - & - & - & - & - & - & - \\
\hline$\varepsilon_{2}$ & Right & $5.88 \%$ & $6.16 \%$ & $6.32 \%$ & $6.35 \%$ & $6.22 \%$ & - & - & - & - & - & - & - & - & $\%$ \\
\hline$w_{m}$ & Right & - & - & - & - & -2.14 & -2.10 & -2.01 & -1.91 & -1.85 & -1.67 & -1.51 & -1.45 & -1.29 & w \\
\hline$\beta_{1}$ & Right & - & - & - & - & 1.59 & 1.69 & 1.75 & 1.78 & 1.84 & 1.84 & 1.83 & 1.92 & 1.94 & - \\
\hline 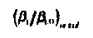 & Right & - & - & - & - & 1.00 & 1.06 & 1.10 & 1.12 & 1.16 & 1.16 & 1.15 & 1.20 & 1.22 & - \\
\hline$\left.\left(\beta_{/} / \beta_{0}\right)_{u}\right)_{u}$ & Right & - & - & - & - & 1.00 & 1.13 & 1.27 & 1.39 & 1.50 & 1.63 & 1.76 & 1.84 & 1.99 & - \\
\hline$\varepsilon_{1}$ & Right & - & - & - & - & $3.92 \%$ & $3.85 \%$ & $3.70 \%$ & $3.49 \%$ & $3.38 \%$ & $3.08 \%$ & $2.77 \%$ & $2.70 \%$ & $2.43 \%$ & $\%$ \\
\hline$\Sigma_{w}$ & Global & 13.4 & 7.0 & 12.0 & 11.3 & -8.8 & -12.2 & 9.2 & -2.7 & 9.3 & 10.4 & 1.9 & 5.2 & 15.8 & $w$ \\
\hline$\Sigma_{t r k}$ & Global & 289.3 & 336.3 & 371.2 & 408.4 & 451.8 & 426.7 & 398.1 & 366.5 & 317.0 & 269.7 & 228.5 & 343.1 & 245.0 & w \\
\hline$\% \Delta_{\text {nlktr }}$ & Global & $4.63 \%$ & $2.10 \%$ & $3.22 \%$ & $2.77 \%$ & $-1.95 \%$ & $-2.87 \%$ & $2.32 \%$ & $-0.73 \%$ & $2.93 \%$ & $3.84 \%$ & $0.85 \%$ & $1.51 \%$ & $6.45 \%$ & $\%$ \\
\hline$\% \Delta_{*}$ & Global & $-3.12 \%$ & $-0.72 \%$ & $-1.88 \%$ & $-1.76 \%$ & $-0.76 \%$ & $-0.55 \%$ & $0.02 \%$ & $-0.65 \%$ & $-0.32 \%$ & $1.27 \%$ & $0.18 \%$ & $0.74 \%$ & $1.98 \%$ & $\%$ \\
\hline
\end{tabular}


Table B.9. Values for Various Enthalpy Flux Ratios

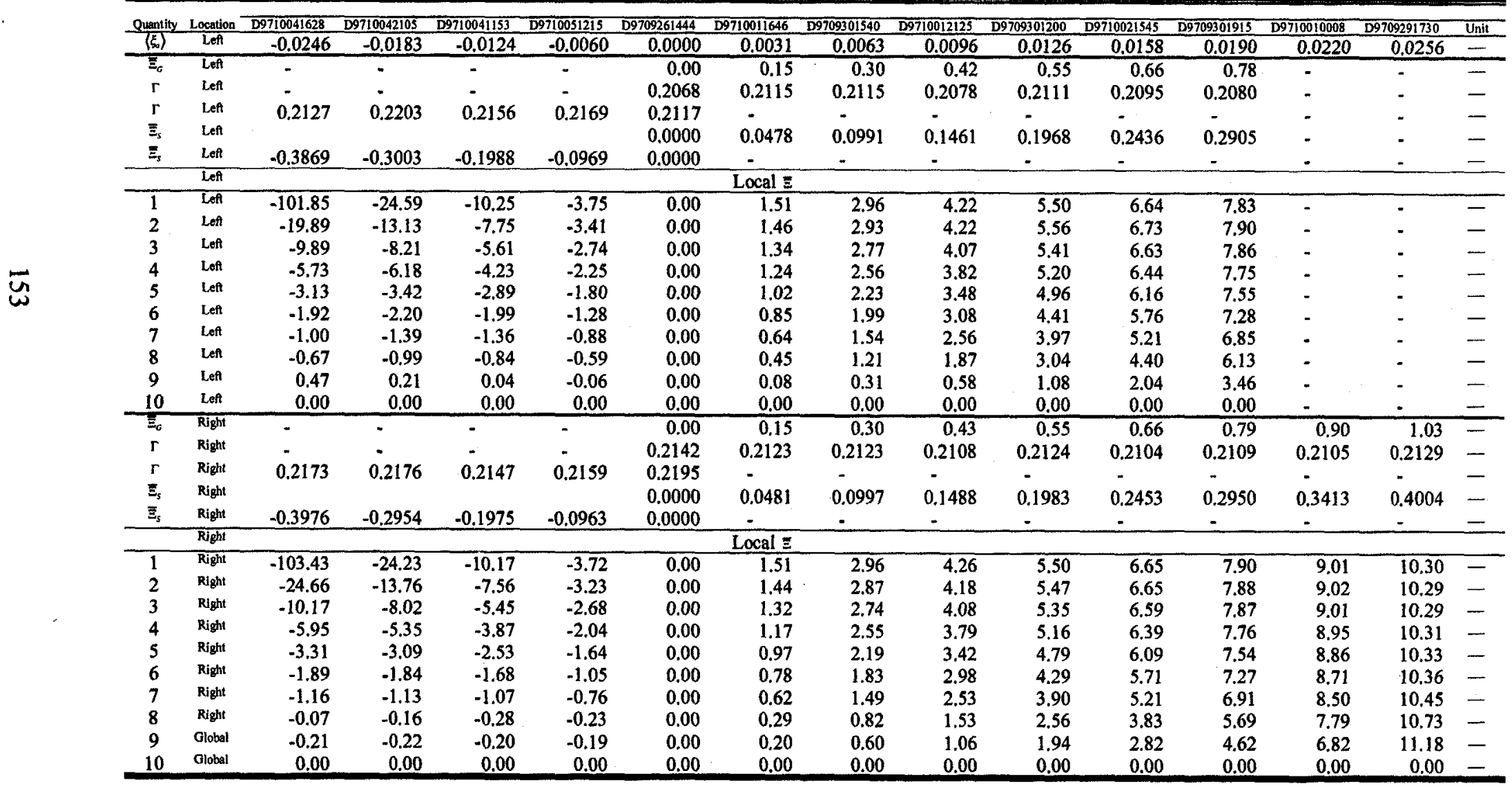




\section{APPENDIX C}

\section{LINEAR ACOUSTIC MODEL}

The linear acoustic model computes the one-dimensional wave equation for a series of coupled acoustic volumes forming a system of nonlinear equations. The Powell-hybrid method solves this system of equations using the targets and guesses that the user selects to manipulate the differences between the targets and results to zero. The continuity of $p_{1}$ and $U_{1}$ links the boundaries of the acoustic volumes in a manner similar to a computation presented in Chapter 3. The algorithms used in this model are found in Ward and Swift (1997). The assumptions used for the implementation of applied flow in this model are found in Appendix A. The compiled code and users' manual (with applied flow implementation) are on the World Wide Web at http://www.lanl.gov/thermoacoutics/. Figure 34 shows a scale drawing of the open thermoacoustic refrigerator with the acoustic volumes used to represent the refrigerator in the linear acoustic model. Following Figure 34 is a typical linear acoustic model run file with the input information on the left column and the computational results on the right column. 


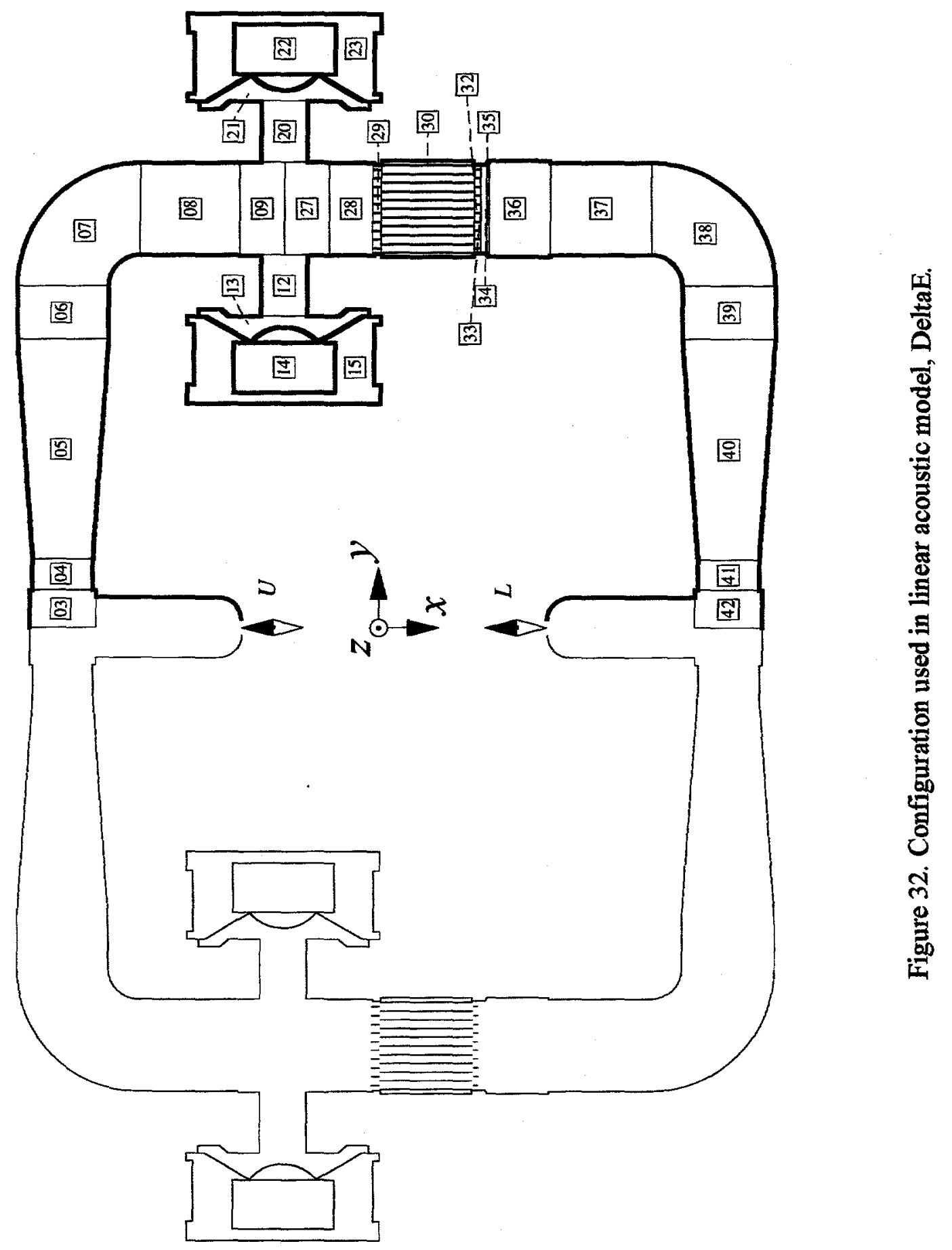


segment defines conditions at upper pressure node.

segment defines magnitude of applied

flow rate through resonator.

Bacmi Preseure Hode at Tee

3.2272E+05 a Wan $P$ Pa 93.938 b Ereq. Hz 307.89 c $T$-beg $K$ 0.0000 d |pleo pa $90.000 \quad$ Eh(p)0 des $0.1959+10100 \quad \mathrm{~m} / \mathrm{3} / \mathrm{z} G$ -272.43 g Eh(D)0 dag $a$ 0.920 hear eas type idan solid type

mensiow man flow $(+R \leqslant-R)$

$3.870 \alpha-03=0=\mathrm{m}=3 / \mathrm{s}$

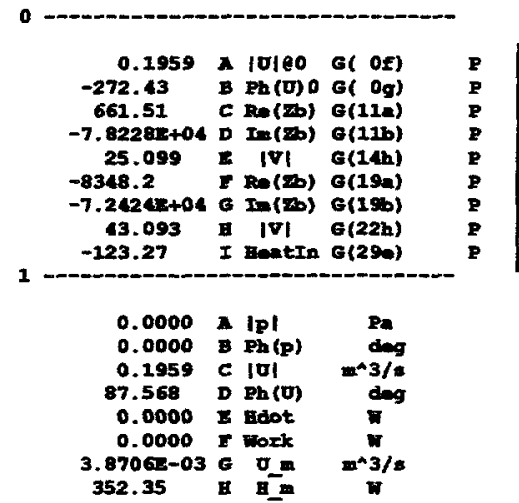

- Cas type

Detreen properties:

$$
\text { ! }
$$

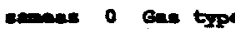

idavel solid type

algorithm used found

in Ward and Swift

1997), Equations

VI.1 and VI.2

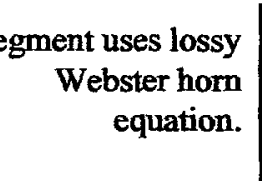

\section{Duct 4.40" $\times 2$. 2n $^{\prime \prime}$ 0.3511 a drae $6.35002-02$ e Inength \\ vinear 0 cas type \\ ideal solid type \\ Isopolet Duet $4.00^{n} \times 2.0^{\circ}$ \\ 8.0992R-03 a Aran $\quad=\wedge 2$ 0.3190 barim \\ 5.08002-02 e Inegth

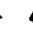

anoss

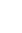

Gas typ

Solid type

Isocone Diffuear $8^{\circ}-14.5^{\circ}$

8.09925-03 a MreaI 0.3190 b ParimI 0.3692 e Inength 1.8430E-02 d Arear 0.4813 - Parin

chomas 0 Gas type

ideat solid type

rsoduct Duet $6.03^{\prime \prime} \times 3.5^{\prime \prime}$

$1.8430 \mathrm{~s}-02 \times$ Area

0.4913 b Peric

8.8900z-02 $c$ Inength

sameas 0 Gas type
ideal

Isoduet Ibow 6.03" IDx 8.0"

$1.84305-02$ area 0.4813 b Perim

0.2020 c Inongth

$=$ (11)

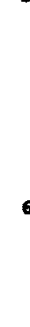

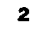

\section{$1.6667 \mathrm{a} g \mathrm{gm}$}

787.57 \& $a$ a

0.8672 C rho $\mathrm{kg} / \mathrm{m}+3$

021.9 o tho $\mathrm{kg} / \mathrm{ma}$

0.1321 ह $\frac{E P}{\mathrm{x}} \mathrm{J} / \mathrm{kg} / \mathrm{x}$

2.119315-05 F m kg/s/m

$3.24795-03$ E bota $1 / \mathrm{R}$

4.13275-04 E deltax

2.8778I-04 I deltars

$1.0000 \mathrm{~s}+08 \mathrm{I}$ sho $\mathrm{kg} / \mathrm{m}^{\wedge} 3$

$1.00002+08 \mathrm{~K} \in \mathrm{J} / \mathrm{kg} / \mathrm{k}$

$1.00001+08$ I $x=\mathrm{m} / \mathrm{s} / \mathrm{k}$

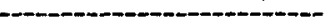

$\begin{array}{lll}652.08 & A & |P| \\ -2.7284 & B & P\end{array}$

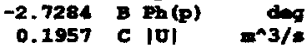

$\begin{array}{lll}0.1957 & C \text { IVI } \\ 87.569 & \text { D Fh(U) }\end{array}$

0.3307 E Edot

-0.3307 I Wozk

-0.3307 G HentIn

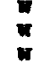

$1283.1 \quad \mathrm{a}|\mathrm{p}|$

-2.7426 B Ph(P)

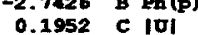

87.570 D Ph(U)

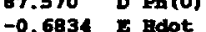

-0.6834 Work -0.3527 Gentin

Pe

ieg

3/:

deg

4239

-2.7041 B Ph(p)

0.1785 c $|0|$

87.619 D $\mathrm{Ph}$ (U)

-1369 rdot

2.1369 b fol

-1.4535 G Bant

it

-

\begin{tabular}{|c|c|c|}
\hline $\begin{array}{c}1672.3 \\
-2.6931 \\
0.1705 \\
87.644 \\
-2.3437 \\
-2.3437 \\
-0.2068\end{array}$ & 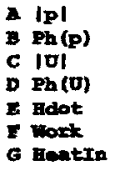 & 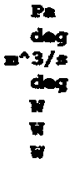 \\
\hline $\begin{array}{c}5574.8 \\
-2.6680 \\
0.1490 \\
87.719 \\
-2.8110 \\
-2.8110\end{array}$ & $\begin{array}{l}\text { A }|p| \\
\text { B Ph(p) } \\
\text { C |O| } \\
\text { D Fh(o) } \\
\text { I Hdot } \\
\text { I noxk }\end{array}$ & 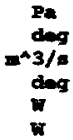 \\
\hline
\end{tabular}

summary of targets and guesses for compuation upstream gas properties and properties of stack plate at that temperature. model treats elbows as straight ducts. 


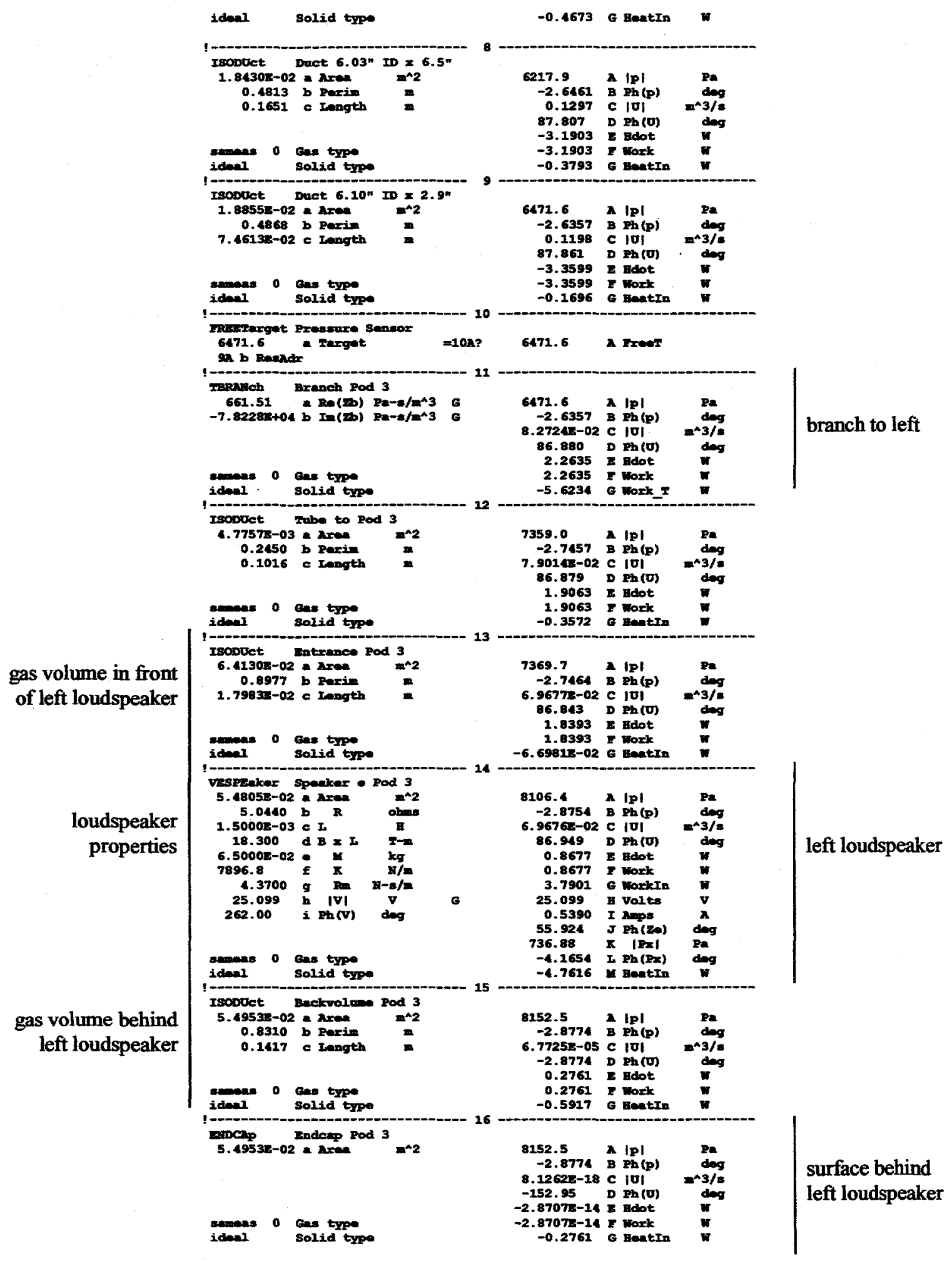




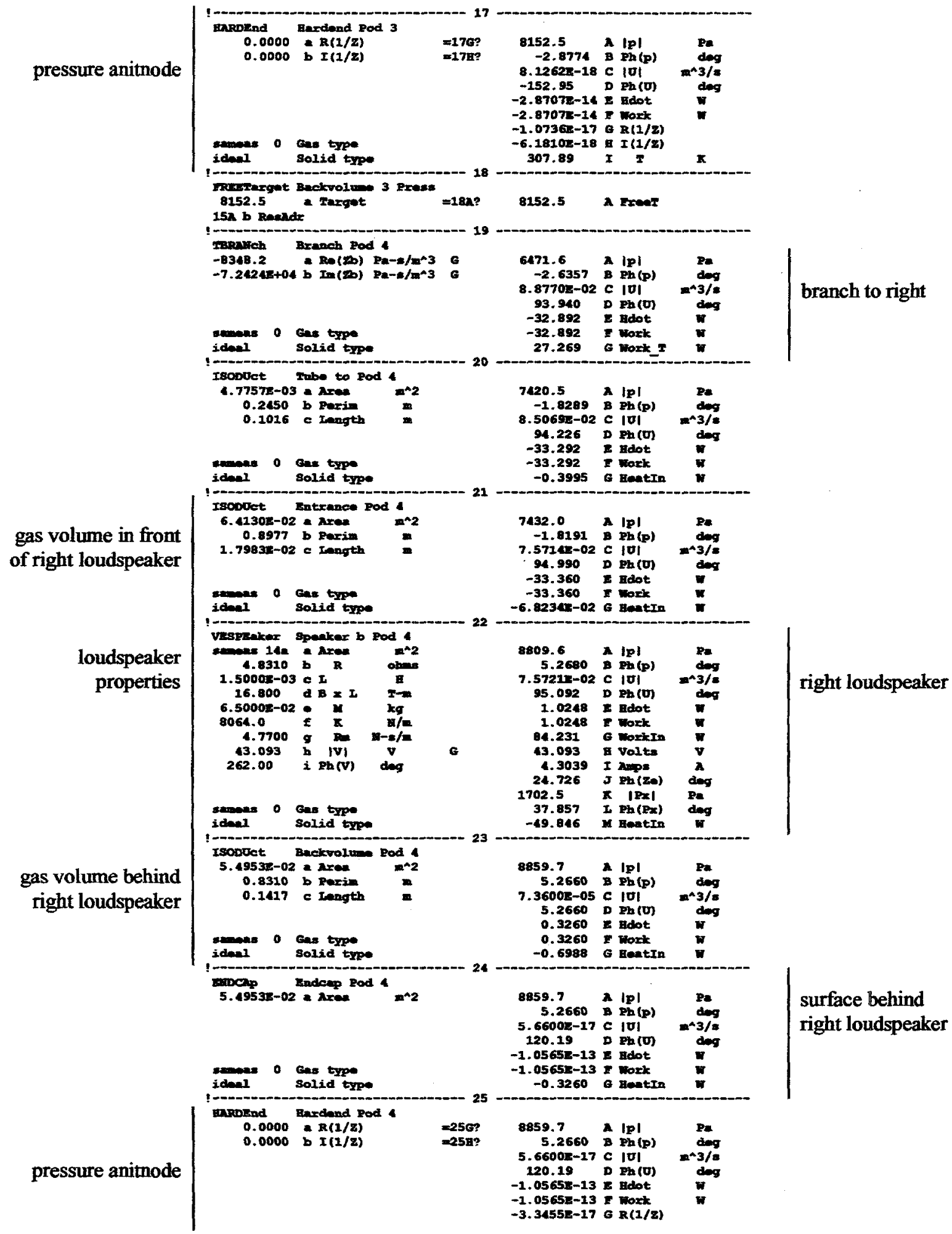




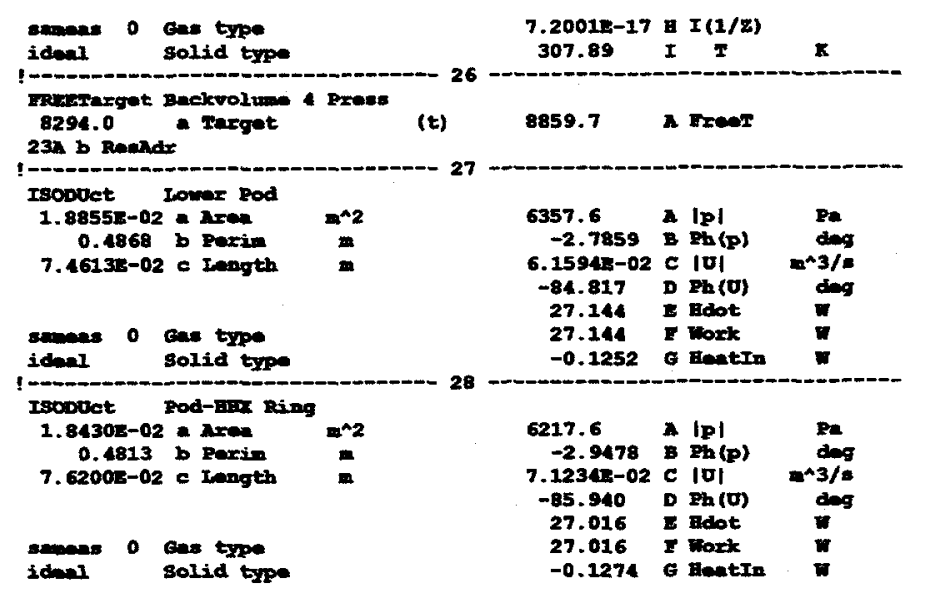

heat exchanger algorithm believed accurate within a factor of 2

target to fix stack end temperature

\section{expet Bot Bout Irechanger}

1.72118-02 a Area 0.5227 b canin/z

$1.27005-02 \&$ Iength

$6.35000-04$ d Y0

-123.27 Hati

307.94 Ist-T

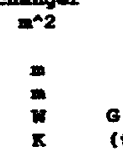

emens 0 Gas type

copper solid type

sworket Rectangular stack

2.0175- -02 axea

0.7050 b cana/a

0.1524 e longti

t.06405-04 d

1.18115-04 Iplate

$6.35000-03$

sarent: 0 cas type

Ifererarget last stack rC

300.67 arget $=3119$

tr.

I- Dxast Cold Heat Erchanger

1.72118-02 a Mrea

0.9300 b Gank/a

$1.04145-03$ d 70

$1.04145-03$ d $\mathrm{Y}^{0}$

18.417 EcatI
298.00 Iat-r

$0.920 h a n$ cas type

copper solid type

mosoust CRx Opatrean Duct

1.7211:-02 area

0.4651 b Perim

9.7155s-03 a Inength

0.0000 d Sxough

sanes 0 Gas type
idenel solid type

idanel Solid type

IsobUet Cix Downstram Dact

1.72115-02 = Arans an

0.4651 b Pari

5. 9055z-03 a rangth

anane o Gas type

idanl

solid type
29

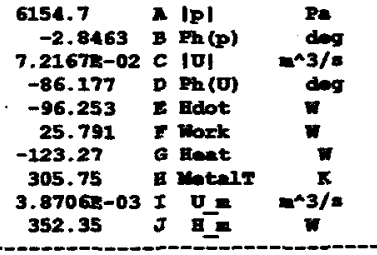

5584.9 |P| Pa

$9.09631-02$ c $|0|$ ma

-88.943 D Ph(J) deg

$-23.024:$ Hdot

$\begin{array}{rl}-23.024 & \text { E ndot } \\ 2.7506 & \mathbf{r} \text { laxk }\end{array}$

307.89 \& F-beg

300.67 I 7 -end $x$

-23.040 I stketirk

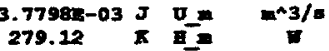
31

300.67 A Prent 32

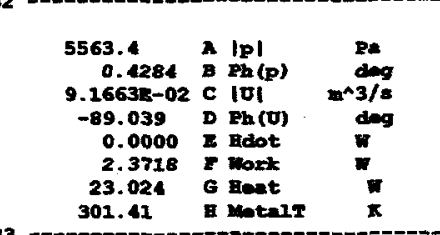

source of electrical heat used to control stack end conditions 


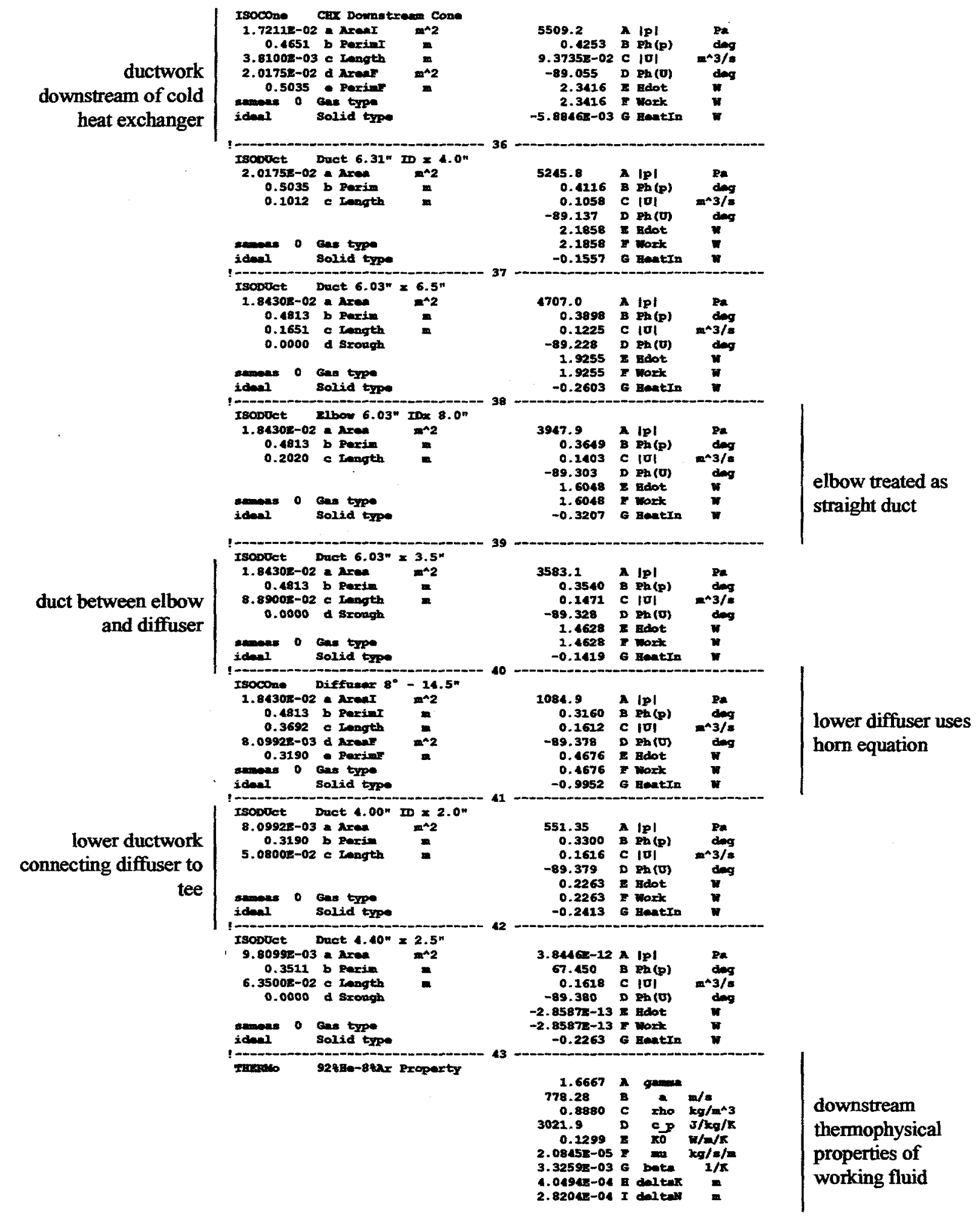




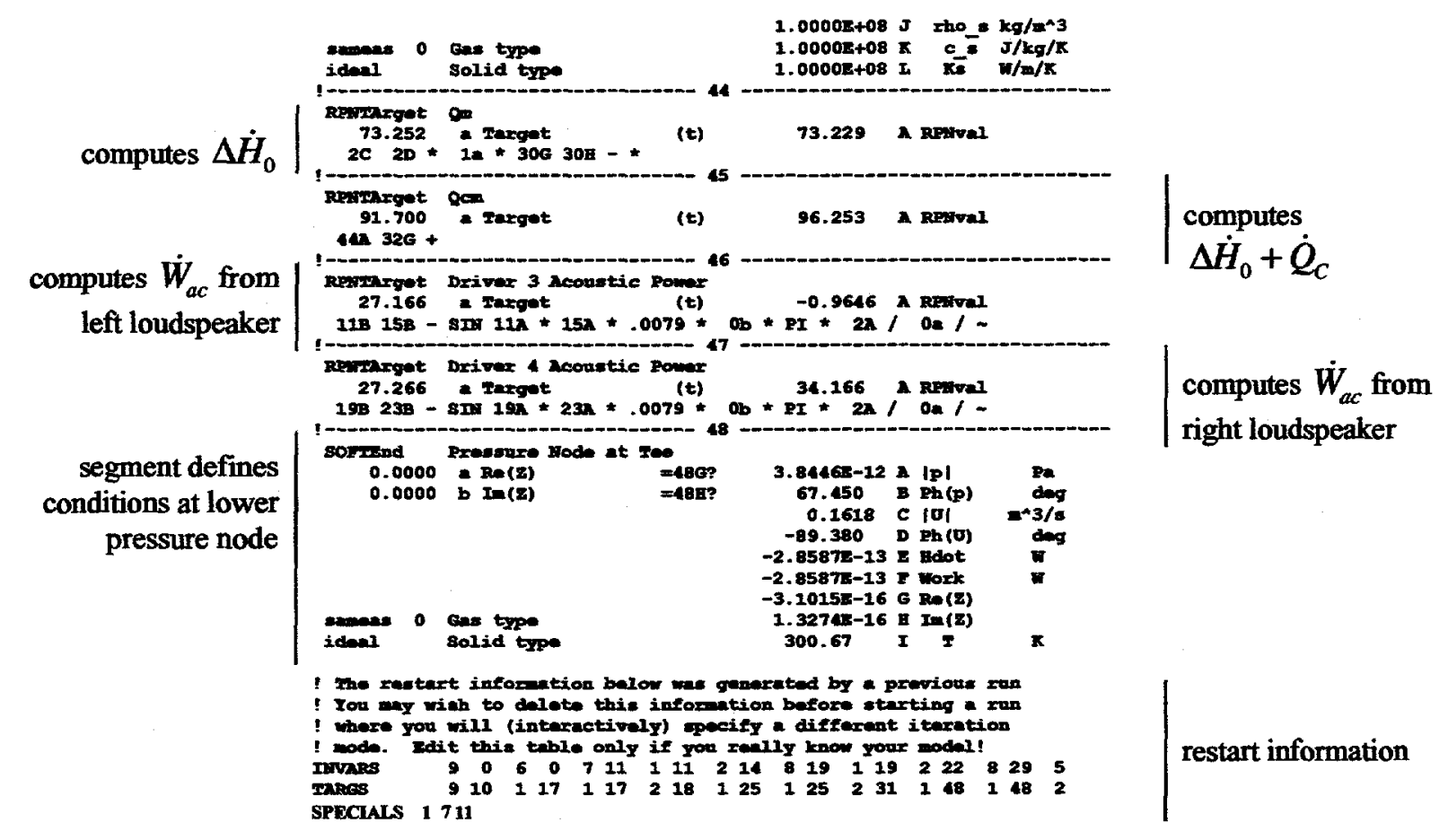




\section{ENDNOTES}

[1] JBL Professional (Northridge, CA 91329) Model 2206H/J $300 \mathrm{~mm}$ Low Frequency Transducer

[2] Omega Engineering (Stamford, CT 06907) Model KQSS-1 16(U)

[3] Cole-Parmer Instrument Company (Vernon Hills, IL 60661) Model N064-63 (ST)

[4] Hewlett Packard (Palo Alto, CA 94304) Model HP33120A

[5] MTI Instruments (Latham, NY 12110) Model Fotonics 1000 with 3812 module

[6] JBL Professional (Northridge, CA 91329) Model 2206H/J 300 mm Low Frequency Transducer

[7] Hewlett Packard (Palo Alto, CA 94304) Model 35665A

[8] ElectroScientific Industries (Portland, OR 97229) Model PVB 300

[9] Hewlett Packard (Palo Alto, CA 94304) Model 35665A Installation and Verification Guide pp. 1-7.

[10] Omega Engineering (Stamford, CT 06907) Model TMQSS-020(U)

[11] Fuji Blowers bought at W. W. Granger (Denver, CO 80223) Model $4 Z 753$

[12] Meriam Instrument (Cleveland, OH 44102) Model 50MW20-1 1/2

[13] Wallace and Tiernan (Vineland, NJ 08360) Model FA-141

[14] Endevco (San Juan Capitrano, CA 92675) Model 8510B-2 
[15] Keithley (Solon, OH 44139) Model 175 Multimeter

[16] Omega Engineering (Stamford, CT 06907) Model KQSS-116(U)

[17] Ashcroft (Costa Mesa, CA 92626) Model 8437

[18] Meriam Instrument (Cleveland, $\mathrm{OH} 44102)$ Model 50MW20-1 1/2 Installation and Operating Instructions.

[19] Keithley (Solon, OH 44139) Model 175 Multimeter

[20] This value is absolute limit of error found on p. Z-168 Omega Temperature Handbook, Vol. 29. Omega Engineering (Stamford, CT 06907).

[21] This value is absolute limit of error found on p. Z-188 Omega Temperature Handbook, Vol. 29. Omega Engineering (Stamford, CT 06907).

[22] Endevco (San Juan Capitrano, CA 92675) Model 8510C-50

[23] Omega Engineering (Stamford, CT 06907) Model HH21

[24] Omega Engineering (Stamford, CT 06907) Model TMQSS-020(U)

[25] Endevco (San Juan Capitrano, CA 92675) Model 8510B-5

[26] EG\&G Instruments Corporation (Princeton, NJ 08543) Model 5301

[27] Keithley (Solon, OH 44139) Model 175 Multimeter

[28] Wallace and Tiernan (Vineland, NJ 08360) Model FA-129

[29] This value is absolute limit of error found on p. Z-188 Omega Temperature Handbook, Vol. 29. Omega Engineering (Stamford, CT 06907). 


\section{REFERENCES}

Allen, P. C., W. R. Knight, D. N. Paulson, and J. C. Wheatley, Proc. Natl. Acad. Sci. USA, 77, 39 (1980).

Arnott, W. P., H. E. Bass, and R. Raspet "General formulation of thermoacoustics for stacks having arbitrarily shaped pore cross sections," J. Acoust. Soc. Am. 90 (1991): 3228.

Backhaus, S. and G.W. Swift, "A thermoacoustic Stirling heat engine," Nature 399, 335338 (1999).

Bejan, A. Advanced Engineering Thermodynamics, (Wiley, 1997).

Crunkleton, J. A., J. L. Smith, and Y. Iwasa, Adv. Cryogenic Eng., 33, 809 (1988).

Feldman, K. T., "Review of the literature on Rijke thermoacoustic phenomena," J. Sound Vib. 7.71 (1968).

Garrett, S. L., and T. J. Hofler, "Thermoacoustic refrigeration," ASHRAE Journal 34.12 (1992): 28.

Hayek, F. A., The Counter-Revolution of Science: Studies in the Abuse of Reason, (London, Collier-Macmillan, 1952).

Hofler, T. J., Thermoacoustic Refrigerator Design and Performance, Ph.D. Dissertation: University of California, San Diego: 1986. 
Kline, S. J., and F. A. McClintock, "Describing Uncertainties in Single-Sample Experiments," Mechanical Engineering, February 1953.

Landau, L. D., and E. M. Lifshitz, Fluid Mechanics, (London: Pergamon Press, 1987).

Landsberg, P. T. “Cool Sounds,” Nature 394, 623-624 (1998).

Olson, J. R., and G. W. Swift, "Similitude in thermoacoustics," J. Acoust. Soc. Am. 95 (1994): 1405.

Rayleigh, J.W.S., The Theory of Sound, (Dover, 1945) Vol. 2, pp. 231-235.

Reid, R. S., W. C. Ward, and G. W. Swift, "Cyclic Thermodynamics with Open Flow," Physical Review Letters, 80 (1998): 4617.

Rott, N., "Damped and thermally driven acoustic oscillations in wide and narrow tubes," A. Angew. Math. Phys. 20 (1969): 230.

Rott, N., "Thermally driven acoustic oscillations, part iii: Second-order enthalpy flux," $\underline{\mathrm{Z}}$ Angew. Math. Phys. 26 (1975): 43.

Rott, N., "A simple theory of the Soudhauss tube," (Springer-Verlag: Stanford University, CA, 1983).

Swift, G. W., “Thermoacoustic engines," J. Acoust. Soc. Am. 84 (1988): 1145.

Swift, G. W., "Analysis and performance of a large thermoacoustic engine," J. Acoust. Soc. Am. 92 (1992): 1563.

Swift, G. W. Physics Today, 48, 22 (1995). 
Swift, G.W., "Thermoacoustics with mean flow derivation (fragment)," (Personal communication with R.S. Reid, 1997).

Swift, G. W., R. S. Reid, and W. C. Ward, "Method and Apparatus for Adapting Steady Flow with Cyclic Thermodynamics," US Patent Application S-89,626, December 1998.

van Wylen, G. J. and R. E. Sonntag, Fundamentals of Classical Thermodynamics, (Wiley, New York, 1965).

Ward, W. C. and G. W. Swift, J. Acoust. Soc. Am., 95, 3671 (1994).

Ward, W.C. and G. W. Swift (1997) "Design Environment for Low-Amplitude Thermoacoustic Engines," Version 4.0. LA-CC-93-8, available at http://www.lanl.gov/thermoacoustics/.

Yazaki, T., A. Tominaga, and Y. Narahara, Cryogenics, 19, (1979): 393-396.

Zinn, B. T., in Advanced Combustion Methods, edited by F. J. Weinberg, (Academic Press, London, 1986) p. 113. 


\section{VITA}

Robert Stowers Reid was born Saturday, May 20, 1961 in Kansas City, Missouri the son of Robert Edward Reid and Geraldine Stowers Reid. He attended public school in Omaha, Nebraska and graduated from Northwest High School in June 1979. He entered the US Military Academy in July 1979 and there completed seven semesters of course work, majoring in Nuclear Engineering. In July 1983, he was granted a medical discharge from the US Army. In August 1983, he enrolled at the University of Nebraska, Lincoln where he received a BS in Mechanical Engineering in December 1984. He attended Iowa State University, Ames, Iowa from January 1985 to August 1986, and received an MS in Mechanical Engineering in December 1986. M. B. Pate and A. E. Bergles served as comajor professors for his MS thesis "Augmented in-tube evaporation of Refrigerant 113."

In August 1986, he became a Technical Staff Member at Los Alamos National Laboratory. While at Los Alamos he has designed and analyzed heat transfer and fluid flow systems for both the terrestrial and space environments. In the late 1980s he coauthored a report on advanced space power heat rejection concepts for the US Department of Energy's Multi-Megawatt program. In 1989 he designed, built, and tested a gas-loaded cryogenic heat pipe to thermally switch a $\mathrm{YBa}_{2} \mathrm{Cu}_{3} \mathrm{O}_{7}$ superconductor for the Electric Power Research Institute. From 1989 to 1992, he was the lead thermal engineer for the ALEXIS satellite project. Concurrently from 1990 to 1992, he was the development engineer for a subatomic particle detector electronic cooling system at the Superconducting Super Collider in Dallas, Texas. From 1991 to 1992 he also supported the NASA Langley Research Center sponsored National Aerospace Plane leading edge cooling program.

During late 1992 and 1993 he attended the Georgia Institute of Technology sponsored by Los Alamos National Laboratory's Advanced Study Program. From 1994 to 1995, he worked with DuPont in Wilmington, Delaware to develop an improved method to melt spin Nylon-6,6 fibers. He is a licensed professional engineer in the State of New Mexico. Reid now develops components for new propulsion system concepts for the US Navy, NASA Marshall Space Flight Center, and NASA Langley Research Center. His immediate (and supportive) family consists of his parents, brother Doug, sister-in-law Kim, niece Jennifer Marie (born 1993), and nephew Matthew David (born 1997). 